

Bill, R., Zehner, M. L. (Hrsg.) GeoForum MV 2020 Geoinformation als Treibstoff der Zukunft 



\title{
GeoForum MV 2020 - \\ Geoinformation als Treibstoff der Zukunft
}

\author{
Bill, R., Zehner, M. L. (Hrsg.)
}




\section{Verein der Geoinformationswirtschaft Mecklenburg-Vorpommern e.V.}

\section{Vorstand \\ Lise-Meitner-Ring 7 \\ D-18059 Rostock}

Dieses Werk ist lizenziert unter einer Creative Commons "Namensnennung - Nicht-kommerziell - Weitergabe unter gleichen Bedingungen" 4.0 International 4.0 (CC BY NC SA).

Der Text der Lizenz ist unter https://creativecommons.org/licenses/by-nc-sa/4.0/ abrufbar. Eine Zusammenfassung (kein Ersatz) ist nachlesbar unter: https://creativecommons.org/ licenses/by-nc-sa/4.0/legalcode

\section{ISBN 978-3-95545-337-4}

Bibliografische Information der Deutschen Nationalbibliothek

Die Deutsche Nationalbibliothek verzeichnet diese Publikation in der Deutschen Nationalbibliographie; detaillierte bibliografische Daten sind im Internet über http://dnb.d-nb.de abrufbar.

Das Werk einschließlich aller seiner Teile ist urheberrechtlich geschützt. Jede Verwertung außerhalb der engen Grenzen des Urheberrechtsgesetzes ist ohne Zustimmung des Verlages unzulässig und strafbar. Das gilt insbesondere für Vervielfältigungen, Übersetzungen, Mikroverfilmungen und die Einspeicherung und Verarbeitung in elektronischen Systemen.

Veröffentlicht im GITO Verlag 2020

Gedruckt und gebunden in Berlin 2020

(c) GITO mbH Verlag Berlin 2020

GITO mbH Verlag

für Industrielle Informationstechnik und Organisation

Kaiserdamm 23

14059 Berlin

Tel.: +49.(0)30.41938364

Fax: +49.(0)30.41938367

E-Mail: service@gito.de

Internet: www.gito.de
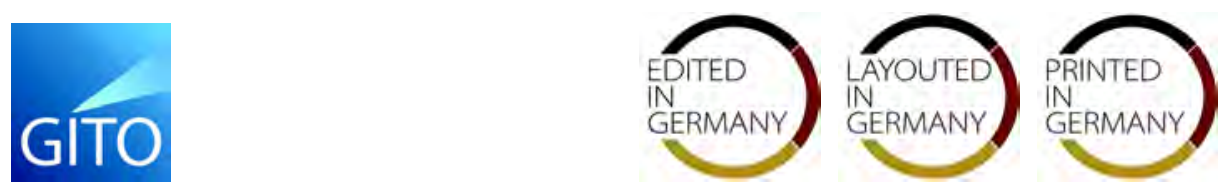
Bill, R., Zehner, M. L. (Hrsg.)

GeoForum MV 2020 -

Geoinformation als Treibstoff der Zukunft 



\section{GE):MV}

\section{GeoForum MV 2020}

\section{Geoinformation als Treibstoff der Zukunft}

Tagungsband zum 16. GeoForum MV

www.geomv.de/geoforum

Warnemünde, 20. und 21. April 2020

Bildungs- und Konferenzzentrum des Technologieparks Warnemünde

AED SICAD

by VertiGIS"
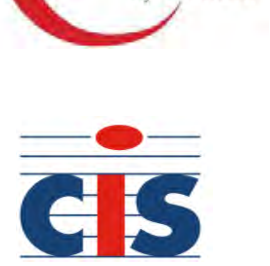
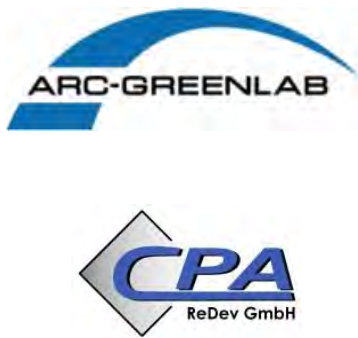
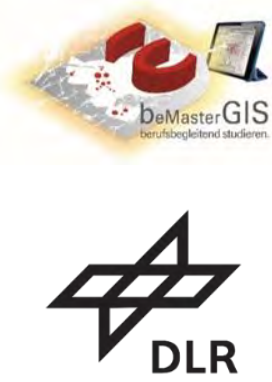

DVZ Datenverarbeitungszentrum Mecklenburg-Vorpommern $\mathrm{GmbH}$

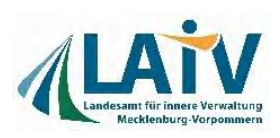





\section{Veranstalter}

GeoMV e.V.

Verein der Geoinformationswirtschaft Mecklenburg-Vorpommern e.V.

Lise-Meitner-Ring 7, 18059 Rostock

www.geomv.de

\section{Herausgeber/Redaktion}

Prof. Dr.-Ing. Ralf Bill

Professur für Geodäsie und Geoinformatik

Agrar- und Umweltwissenschaftliche Fakultät, Universität Rostock

Justus-von-Liebig-Weg 6, 18059 Rostock

www.auf.uni-rostock.de/professuren/a-g/geodaesie-und-geoinformatik/

Dipl.-Ing. M.Sc. Marco Lydo Zehner

DVZ Datenverarbeitungszentrum M-V GmbH

Lübecker Straße 283, 19059 Schwerin

www.dvz-mv.de

\section{Lektorat/Satz}

Dr. Grit Zacharias, www.lektorat-zacharias.de

\section{Aussteller und Sponsoren}

- AED-SICAD GmbH

- ARC-GREENLAB GmbH

- beMasterGIS

- $\mathrm{CiS} \mathrm{GmbH}$

- CPA ReDev GmbH

- DLR Deutsches Zentrum für Luft- und Raumfahrt

- DVZ Datenverarbeitungszentrum M-V GmbH

- LAiV M-V / Amt für Geoinformation, Vermessungs- und Katasterwesen 



\section{Vorwort}

Unter dem Motto „Geoinformation als Treibstoff der Zukunft“ findet am 20. und 21. April 2020 das 16. GeoForum MV im Technologiepark Warnemünde, Rostock statt. Die große Vielfalt an bereits heute verfügbaren und zukünftig noch entstehenden Geoinformationen gilt es nachhaltig zu sichern, in ihrer Qualität und Aktualität zu erhalten und in die intensive Nutzung zu überführen. Geoinformation wird uns dabei wie selbstverständlich in Bürgerprozessen, einfachen Fachverfahren und komplexen smarten Umgebungen begegnen, ohne dass wir sie vielleicht explizit so benennen. Geoinformation wirkt daher als Treibstoff einer nachhaltigen Entwicklung der Geoinformationswirtschaft, und dies nicht nur im Land Mecklenburg-Vorpommern.

Das bewährte Format des GeoForum MV beinhaltet Präsentationen von BestPractice-Beispielen, die Darstellung von technisch-wissenschaftlichen Ergebnissen und viele Gelegenheiten zum persönlichen Erfahrungsaustausch: in den Pausen, auf den Ausstellerständen und beim geselligen Get-together im „Teepott" Warnemünde.

Der vorliegende Tagungsband beinhaltet die 17 Fachbeiträge, die sich in technologieorientierte und anwendungsorientierte Themenblöcke aufteilen. Anwendungsbezogene Schwerpunkte sind im aktuellen Jahr u. a. die Themen Sicherheit (vom Katastropheneinsatz bis zur maritimen Überwachung), Land- und Immobilienmanagement sowie dem Beitrag der Geoinformation im Kontext der Daseinsvorsorge. Technische Fokussierung findet hinsichtlich der Einbettung der GIS-Technologie in Prozesse, Virtuellen Welten und Offenheit bezüglich Daten und Nutzung statt. Darüber hinaus schauen wir seit Jahren auch auf die rechtlichen Rahmenbedingungen, in diesem Jahr z. B. auf die Auswirkungen der neuen PSI-Richtlinie und deren Relevanz für Geodaten als „hochwertige Datensätze“.

Die Keynote wird von Franz-Reinhard Habbel, Mitglied des Innovators Club und Mitherausgeber KOMMUNAL, gehalten. Er gehört als Herausgeber von Sachbüchern, Redner und Mitglied zahlreicher Fachgremien zu den führenden Köpfen der Einführung von E-Government und der notwendigen Transformationsprozessen in der Verwaltung durch die Digitalisierung. Seine Keynote befasst sich mit zwei zentralen Aspekten der Kommunen, nämlich Personen und Geodaten. 
Den Autoren sei herzlich für die rechtzeitige Bereitstellung ihrer Beiträge gedankt. Wir bedanken uns bei den Ausstellern, die auf ihren Ständen ihr Produktportfolio darstellen und damit den Treibstoff der Zukunft in Wert setzen. Besonderer Dank gilt darüber hinaus dem Ministerium für Energie, Infrastruktur und Digitalisierung Mecklenburg-Vorpommern für die Übernahme der Produktionskosten des Tagungsbandes, der dadurch unmittelbar mit der Veranstaltung als eBook im Sinne Open Access frei auf der Webseite des GeoMV verfügbar gemacht werden kann.

Wir hoffen, Ihnen auch 2020 wieder ein spannendes und breit gefächertes Tagungsprogramm mit Vorträgen zu aktuellen Entwicklungen in der Geoinformationswirtschaft zu bieten. Wir wünschen uns und Ihnen ein spannendes GeoForum MV 2020, gute Diskussionen und Denkanstöße für die eigenen Arbeiten sowie für die Zusammenarbeit im Rahmen des Vereinswirkens.

Die Organisatoren des GeoForum MV, für den GEOMV e.V.

Prof. Dr.-Ing. Ralf Bill und Marco Lydo Zehner 


\section{Inhalt}

\section{GIS-ANWENDUNGEN}

UMSETZUNG DER BIOTOPTYPENKARTIERUNG IN M-V MIT WEBGIS

PETER KORDUAN, DIRK MÜLLER, ANDRÉ SCHUMANN..................................... 7

RÄUMLICHE SIMULATIONSANALYSE UND VISUALISIERUNG DER

PROFITABILITÄT VON WINDENERGIEANLAGEN IN DEUTSCHLAND

DAVID HENNECKE, CARSTEN CROONENBROECK ….......................................... 13

\section{GIS-TECHNOLOGIE I}

DIE INTERNETPLATTFORM METROPLANER - DAS STANDARDISIERTE

GEOPORTAL DER METROPOLREGION NORDWEST

ROBERT KRÄTSCHMER.

EMBEDDED GIS - DIE EINFACHE BEREITSTELLUNG VON GEODATEN-

FUNKTIONALITÄT IN SACHDATENORIENTIERTEN FACHANWENDUNGEN

MITHILFE DER LOW-CODE-TECHNOLOGIE

ROBERT BEYER

\section{LANDMANAGEMENT UND DASEINSVORSORGE}

NUTZUNG VON GEODATEN FÜR DAS LANDMANAGEMENT - MODELLIERUNG DER LAGEQUALITÄT AUS FREIEN GEODATEN

WINRICH VOß, KENO BAKKER 39

DASEINSVORSORGEATLAS NIEDERSACHSEN - STAND UND AUSBLICK EINES WERKZEUGS ZUR STEUERUNG UND PLANUNG VON

DASEINSVORSORGEEINRICHTUNGEN

MANFRED KLAUS, STEPHAN MÄS, ALEXANDRA WEITKAMP, LARS BERNARD

\section{SICHERHEIT}

VERNETZUNG VON MOBIL UND STATIONÄR GEWONNENEN

SCHADSTOFFWERTEN BEI GROßFLÄCHIGEN ABC-

GEFAHRSTOFFFREISETZUNGEN

CHRISTOPH AVERDUNG.

BRANDSCHUTZBEDARFSPLANUNG IN MECKLENBURG- VORPOMMERN

MITHILFE AMTLICHER GEODATEN

JENNIFER WURLICH, MARCO L. ZEHNER.

EARTH OBSERVATION MARITIME SURVEILLANCE SYSTEM

SERGEY VOINOV, EGBERT SCHWARZ, DETMAR KRAUSE, BJÖRN TINGS 


\section{GEOINFORMATION ALS RESSOURCE}

PSI-RICHTLINIE ANTE PORTAS - GEODATEN ALS „HOCHWERTIGE DATENSÄTZE“

FALK ZSCHEILE.

KOMMUNALE NACHNUTZUNGSMÖGLICHKEITEN VON DATEN DER

LANDESVERMESSUNG MECKLENBURG-VORPOMMERN

JÜRGEN SCHULZ.

UMFRAGE ZUR SITUATION DER WERTSTOFFHÖFE IN DER HANSE- UND

UNIVERSITÄTSSTADT ROSTOCK

FERDINAND VETTERMANN, SAMER NASTAH, LAURINE LARSEN, RALF BILL .95

\section{GIS-TECHNOLOGIE II}

OPEN LOCATION CODE API (OLCA) - WeB-API ZUR NUTZUNG DER „PLUS CODES“ DES OPEN LOCATION CODES

SEBASTIAN GUTZEIT

GEOCACHING ALS AUSDRUCK VON MENSCH-RAUM-BEZIEHUNGEN

MATTHIAS HINZ.

\section{VIRTUELLE WELTEN}

VIRTUELLE TOUREN IM KONTEXT AKTUELLER MIXED-REALITY-

TECHNOLOGIEN

MARKUS BERGER, SARAH WeSTPHAL, PATRICK POSTERT, HANNES HAGEN...... 125

VIRTUELLER ZWILLING FÜR EIN DYNAMISCHES LAGERMANAGEMENT IN

HÄFEN

OLAF POENICKE, ANDREAS HÖPFNER, CHRISTIAN BLOBNER, HEIKO MALY ..... 133

HELGOLAND 3D MIT ARCGIS PRO

STEFAN LIENING

\section{FIRMENDARSTELLUNGEN}

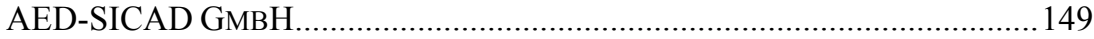

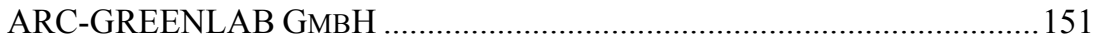

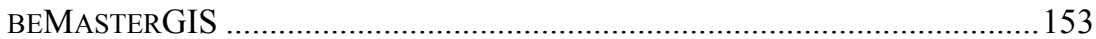

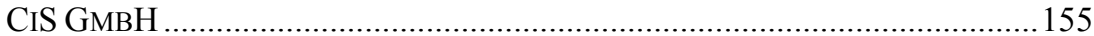

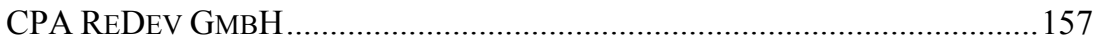

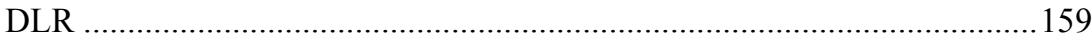

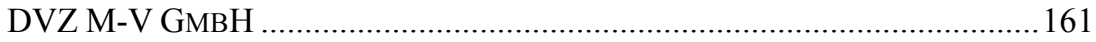

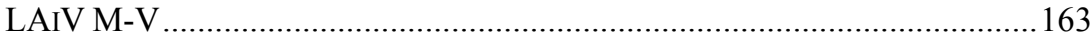


GIS-Anwendungen 



\title{
Umsetzung der Biotoptypenkartierung in $\mathrm{M}-\mathrm{V}$ mit WebGIS
}

\author{
Peter Korduan, Dirk Müller, André Schumann \\ GDI-Service Rostock, UmweltPlan GmbH Stralsund, LUNG M-V \\ peter.korduan@gdi-service.de,dm@umweltplan.de, \\ andre.schumann@lung.mv-regierung.de
}

\begin{abstract}
In diesem Beitrag wird der Aufbau einer WebGIS-Lösung zur Erfassung von Biotoptypen vorgestellt. Die neue Anwendung, die von GDI-Service in Zusammenarbeit mit UmweltPlan für das LUNG MV entwickelt wurde, erfüllt die Anforderungen für eine verteilte Datenerfassung, zentrale Speicherung und abgestufte Arbeitsabläufe mit entsprechenden Zugriffs- und Änderungsrechten. Nach einer Einleitung wird zunächst das Datenmodell der neuen Anwendung vorgestellt sowie auf die Unterscheidung zwischen Daten der Kartierung und der Archivierung eingegangen. Im dritten Abschnitt geht es um den Workflow bei der Erfassung der Daten. Dieser wird in verschiedene Bearbeitungsstände aufgeteilt. Eine besondere technische Herausforderung ist die dynamische Bewertung von LRT-Kartierungen, auf die im vierten Abschnitt eingegangen wird. Schließlich widmen wir uns im letzten Abschnitt der Archivierung der Daten und fassen die Ergebnisse zusammen.
\end{abstract}

\section{$1 \quad$ Einleitung}

Im Jahr 2018 hat das LUNG beschlossen, die Software zur Erfassung von gesetzlich geschützten Biotopen und zur Erfassung und Bewertung von FFHLebensraumtypen (MVBIO5) auf die OpenSource-WebGIS-Lösung kvwmap umzustellen. Die auf VisualBasic6 und dem mdb-Format basierende Software war bereits 20 Jahre alt und konnte technologisch bedingt nur lokal installiert werden. Erfasst und bewertet werden konnten nur die Fachinformationen; Bilder und Geometrien wurden getrennt verwaltet. Dadurch war die Datenzusammenführung von verschiedenen Installationen sehr zeitaufwendig und fehleranfällig. Hinzu kamen Schwierigkeiten bei der Verarbeitung der immer größer werdenden Datenmengen von über 200.000 Datensätzen. Die von GDI-Service in $\mathrm{Zu}-$ sammenarbeit mit UmweltPlan neu entwickelte Lösung baut auf WebGIS- 
Technologien auf, die client-serverbasiert ist, eine zentrale Geodatenbank nutzt und die Bilder und Geometrien mit integriert. Die Erfassung und Bewertung der Daten basiert auf der ,Anleitung für die Kartierung von Biotoptypen und FFHLebensraumtypen M-V“ (LUNG, 2013). Das maßgebende Konzept ist die Erfassung in verschiedenen Erfassungsbögen. Die Inhalte sind so aufgebaut worden, dass eine einfache Erfassung auf Papier im Feld erfolgen kann. Die Informationen, die in den unterschiedlichen Bögen erfasst wurden, enthielten nur textliche räumliche Angaben, waren zum Teil redundant, weil sie aufeinander aufbauen, bedingen sich gegenseitig und mussten eine eindeutige Referenz zu einem Geometrieobjekt haben. Bei der Umstellung auf die WebGIS-Lösung bestand zwar die Forderung, alle Altdaten einzulesen, aber auch die Möglichkeit, das Datenmodell so umzubauen, dass die Erfassung redundanzfrei erfolgen kann.

\section{Datenmodell}

Zur Übernahme der Sachdaten in der Access-Datenbank und der Geometriedaten in den Shape-Dateien wurde ODBC und shp2pgsql eingesetzt. Der Import erfolgte in das Schema import. Dort wurden zunächst auch alle Codelisten und Daten zu Gebietseinheiten und Pflanzenarten abgelegt. Das Datenmodell für MVBIO-Pro besteht aus den Schemata archiv, gebietseinheiten und mvbio. In archiv werden alle historischen Daten abgelegt, die nicht mehr editierbar sind. Die zentrale Entität ist der Erfassungsbogen. Davon abgeleitet sind die verschiedenen Bogenarten Kurzbögen, Grundbögen und Bewertungsbögen, von denen wiederum fünf verschiedene Bewertungsbögen abgeleitet sind. Jeder Bogen hat somit eine Bogenart, die zu einer Kartierebene gehört, und ist einer Kartierkampagne zugeordnet, die in Kartiergebiete unterteilt ist. Die Bogendaten sollen so gespeichert werden, dass sie nicht mehr geändert werden können. Dafür ist es hilfreich, wenn keine Verknüpfungen zu aktuellen Codelisten enthalten sind. Alle Werte aus Codelisten werden als Text in die Archivtabellen übernommen.

Das Schema mvbio enthält hingegen alle Daten von laufenden Kartierungen (Abbildung 1). Kartierungen werden immer Kartiergebieten und diese Kampagnen zugeordnet. Es wird zwar auch erfasst, um welche Bogenart es sich handelt, aber diese Information ist bei der Erfassung nur für die Anzeige des Erfassungsformulars relevant. Alle Kartierungen werden in derselben Tabelle erfasst. Wenn eine Kartierung mit dem Schutzmerkmal ,geschützt nach FFHRichtlinie“ ausgestattet ist, gehört zusätzlich eine Bewertung zur Kartierung. 


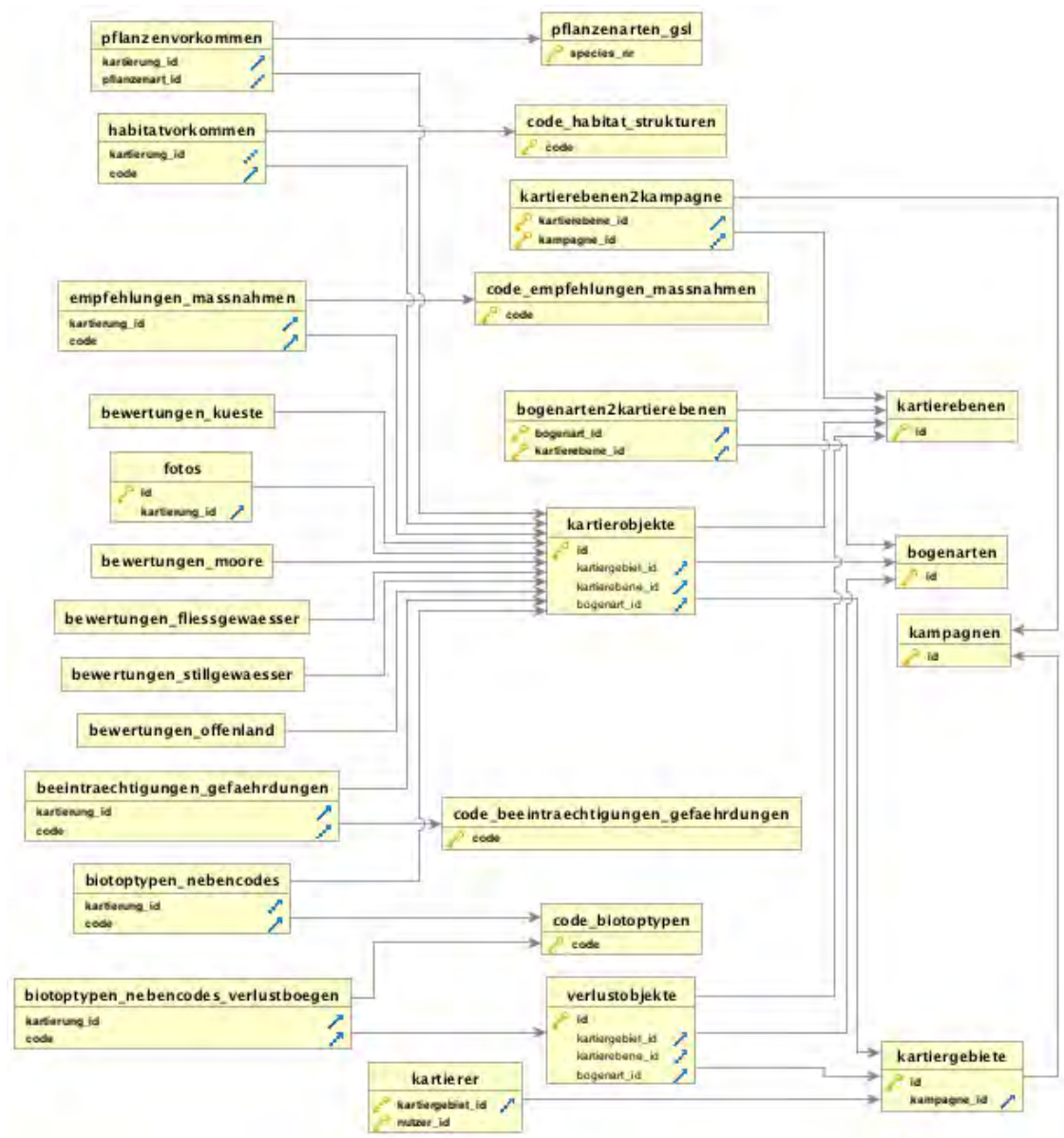

\section{Abbildung 1: Datenmodell im Schema mvbio}

Bei der Erfassung werden die Entitäten Kartierung und Bewertung verwendet und im Archiv zu Grundbogen und Bewertungsbogen. Die Unterscheidung in Kartierung und Bewertung bzw. Grundbogen und Bewertungsbogen ist dem geschuldet, dass die Anzahl der Attribute in der Bewertung sehr groß ist (z. T. über 60 Attribute) und sich auch noch in die Bewertungsarten Fließgewässer, Küste, Moore, Offenland und Stillgewässer unterteilt. Kartierungen und Bewertungen stehen in einer 1:1-Beziehung und die Tabellen „Bewertungsarten“ sind 
von der Tabelle „Bewertung“ abgeleitet. Kartierungen können nur von Nutzern ausgeführt werden, die über die Tabelle „Kartierer“ dem Kartiergebiet zugeordnet sind, in dem die Kartierungen liegen. Nutzer werden also zu Kartierern durch die Zuordnung zu Kartiergebieten. Die Nutzer des Systems sind in einer MySQL-Datenbank enthalten, wo auch die Einstellung der Anwendung hinterlegt sind, und werden über den foreign Data Wrapper postgres_fdw in das Schema mvbio eingebunden. Wenn so ein neuer Nutzer über die Oberfläche angelegt wird, steht er sofort zur Auswahl als Kartierer zur Verfügung.

\section{$3 \quad$ Kartierung}

Für die Kartierung von Biotopen wurde das WebGIS kvwmap eingesetzt. In der Benutzeroberfläche sind Zuständigkeiten festgelegt, die sich in den Nutzergruppen Administration, Auskunft, Kartierung, Koordinierung und Prüfung widerspiegeln.

\begin{tabular}{|l|l|l|l|l|l|}
\hline Bearbeitungsstufe & $\begin{array}{l}\text { Kartierung } \\
\text { eigene KO }\end{array}$ & $\begin{array}{l}\text { Kartierung } \\
\text { frende KO }\end{array}$ & Koordinierung & Prüfung & Auskunft \\
\hline 1 in Erfassung & editierbar & $\begin{array}{l}\text { nur } \\
\text { Geometrie } \\
\text { lesbar* }\end{array}$ & $\begin{array}{l}\text { nur Geometrie } \\
\text { lesbar }\end{array}$ & nicht sichtbar & $\begin{array}{l}\text { nicht } \\
\text { sichtbar }\end{array}$ \\
\hline $\begin{array}{l}2 \text { zur Info } \\
\text { freigegeben }\end{array}$ & editierbar & alles lesbar & alles lesbar & nicht sichtbar & $\begin{array}{l}\text { nicht } \\
\text { sichtbar }\end{array}$ \\
\hline $\begin{array}{l}3 \text { Durch Kartierer } \\
\text { freigegeben }\end{array}$ & alles lesbar & alles lesbar & editierbar & alles lesbar & $\begin{array}{l}\text { nicht } \\
\text { sichtbar }\end{array}$ \\
\hline $\begin{array}{l}4 \text { Durch } \\
\text { Koordinator } \\
\text { freigegeben }\end{array}$ & alles lesbar & alles lesbar & alles lesbar & editierbar & $\begin{array}{l}\text { nicht } \\
\text { sichtbar }\end{array}$ \\
\hline $\begin{array}{l}5 \text { Gesperrt durch } \\
\text { Prüfinstanz }\end{array}$ & alles lesbar & alles lesbar & alles lesbar & alles lesbar & $\begin{array}{l}\text { nicht } \\
\text { sichtbar }\end{array}$ \\
\hline $\begin{array}{l}6 \text { zur Archivierung } \\
\text { freigegeben }\end{array}$ & gelöscht & gelöscht & gelöscht & gelöscht & $\begin{array}{l}\text { nicht } \\
\text { sichtbar }\end{array}$ \\
\hline $\begin{array}{l}7 \text { in Archiv } \\
\text { überführt }\end{array}$ & gelöscht & gelöscht & gelöscht & gelöscht & $\begin{array}{l}\text { Als } \\
\text { Bögen } \\
\text { lesbar }\end{array}$ \\
\hline $\begin{array}{l}8 \text { Bestand } \\
\text { aktualisiert }\end{array}$ & gelöscht & gelöscht & gelöscht & gelöscht & $\begin{array}{l}\text { Im } \\
\text { aktuellen } \\
\text { Bestand }\end{array}$ \\
\hline
\end{tabular}

\section{Abbildung 2: Bearbeitungsstufen von Kartierobjekten}


Der Workflow für die Kartierung sieht zunächst vor, dass der Prüfer die neue Kampagne mit seiner räumlichen Ausdehnung und den darin zu erfassenden Bogenarten festlegt. Die Koordinierung unterteilt diese in Untergebiete und ordnet Nutzer als Kartierer zu. Daraufhin kann der Kartierer ein Kartierobjekt anlegen, welches dann die in Abbildung 2 angegebenen Bearbeitungsstufen durchläuft. Die Koordinierung und Prüfung kann an Kartierobjekte jeweils Kommentare anbringen und über die Bearbeitungsstufe zurückweisen. Die Sichtbarkeiten und Editierrechte sind über den Attributeditor und eine Editiersperre des WebGIS eingerichtet worden. Bei der Eingabe in das Erfassungsformular, welches sich an die Maske des Vorgängerprogramms MVBIO anlehnt (Mordhorst, 2016), werden je nach Bogenart und LRT-Code Felder ein- oder ausgeblendet. Die Prüfung auf Pflichtelemente und Plausibilität wird in einem Datenbanktrigger durchgeführt. Eine besondere Art der Kartierung ist die Erfassung von Verlustobjekten. Diese können nur von bestehenden Bögen aus dem Archiv heraus angelegt werden.

\section{Bewertung}

Kartierungen von Lebensraumtypen können bewertet werden. Im Formular der Bewertung werden anhand der Eintragung direkt die Bewertungsergebnisse A, $\mathrm{B}$ oder $\mathrm{C}$ berechnet und farblich grün, gelb oder rot dargestellt. Am Ende des Bogens werden die Bewertungen zusammengefasst, vom Bearbeiter eingeschätzt und mit einer Bemerkung versehen.

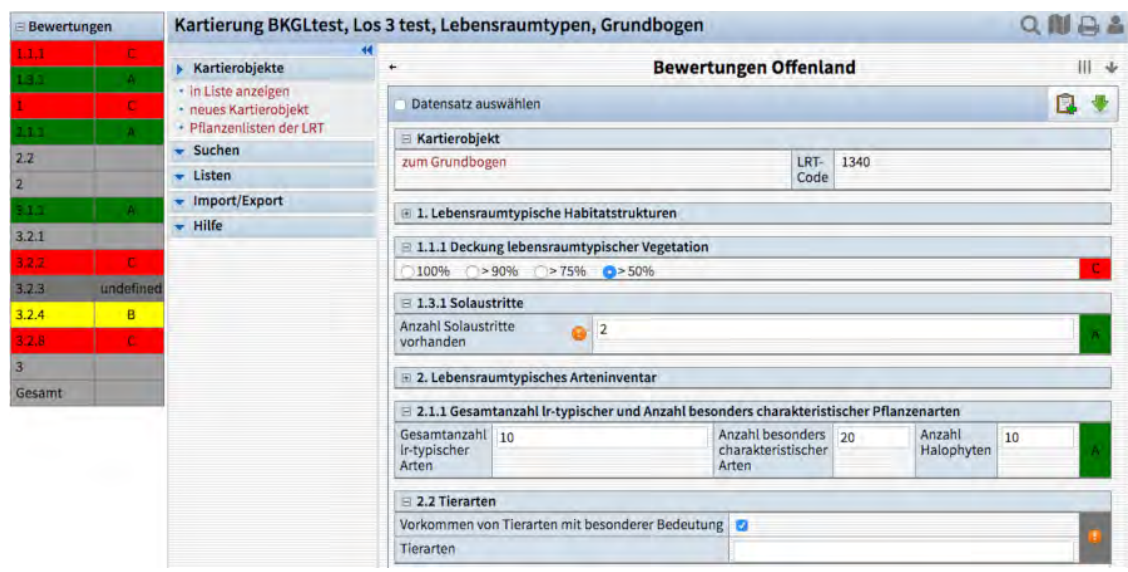


Die Regeln und Formeln für die Bewertung wurden in JavaScript umgesetzt und können durch einen Klick auf die Ordnungsnummer der Bewertung im Browser aufgerufen werden.

\section{$5 \quad$ Archivierung}

Der Übergang von Stufe 6 zu 7 erfolgt über einen Cron-Job im Hintergrund. Bei der Archivierung werden von den Kartierungen im Archiv Bögen angelegt, als PDF-Dokumente erstellt und den Bögen im Archiv zugeordnet. Sind alle Kartierobjekte eines Kartiergebietes in das Archiv übernommen worden, kann der Prüfer das Kartiergebiet in den Bestand übernehmen. Dazu gehen alle im Gebiet bisher aktuellen Bögen unter und die neuen Bögen ersetzen die alten. Es ist also auf eine flächendeckende Kartierung pro Gebiet zu achten. Die Zuordnung zu den Gebietseinheiten Untere Naturschutzbehörden, Gemeinde, Kreis oder Schutzgebieten erfolgt automatisch über Flächenverschneidung. Die Frage der Anpassung der Kartierflächen am Rand der Gebiete ist noch nicht endgültig geklärt. Möglicherweise werden Kartierflächen am Gebietsrand abgeschnitten oder ersetzen auch alte Kartierungen außerhalb der Gebiete. Anhand der aktuellen Bögen lassen sich aus der Datenbank jährliche Biotopverzeichnisse ableiten.

\section{Zusammenfassung}

Mit dem eingesetzten WebGIS kvwmap können die Biotoptypen im Browser kartiert, in einer zentralen Geodatenbank gespeichert, über Rollen im Erfassungsprozess geprüft, kommentiert, archiviert und schließlich in den aktuellen Bestand übernommen werden. Die Verknüpfung von Geometrie- mit Sachdaten erleichtert die Verarbeitung und Prüfung der Daten erheblich und die Biotopverzeichnisse können automatisiert abgeleitet werden.

\section{Literatur}

LUNG, 2013: Anleitung für die Kartierung von Biotoptypen und FFH-Lebensraumtypen in Mecklenburg-Vorpommern, Schriftenreihe des Landesamtes für Umwelt, Naturschutz und Geologie 2013, Heft 2, Stand 2013.

Mordhorst, H.-J. (2016): Biotopkartierung Mecklenburg-Vorpommern MVBIO 5, Technisches Handbuch, Version 5.4.3, Stand 1.10.2016. 


\title{
Räumliche Simulationsanalyse und Visualisierung der Profitabilität von Windenergieanlagen in Deutschland
}

\author{
David Hennecke ${ }^{1}$, Carsten Croonenbroeck ${ }^{2}$ \\ ${ }^{1}$ Universität Rostock, Professur für Geodäsie und Geoinformatik, Justus-von- \\ Liebig-Weg 6, 18059 Rostock, david.hennecke@uni-rostock.de \\ ${ }^{2}$ Universität Rostock, Professur für Agrarökonomie, \\ Justus-von-Liebig-Weg 7, 18059 Rostock, carsten.croonenbroeck@uni- \\ rostock.de
}

\begin{abstract}
Im Zuge einer Simulationsanalyse hinsichtlich des Vergütungssystems des überarbeiteten Erneuerbare-Energien-Gesetzes (EEG) aus dem Jahr 2017 wird eine Webapplikation entwickelt, um die entstandenen Daten zu visualisieren sowie öffentlich zugänglich und nutzbar zu machen. Ziel ist es, eine Oberfläche zu schaffen, auf der eine WindkraftStandortanalyse auf Basis von Windgeschwindigkeiten, Turbinen-HöhenKombinationen, Korrekturfaktoren i. S. d. § 36h EEG Standorterträgen und gewichteten Standorterträgen durchgeführt werden kann. Zusätzlich bietet die Applikation klassische GIS-basierte Analysetools, welche Standortanalysen hinsichtlich nahe gelegener Infrastrukturen (z. B. Gebäude) und Naturschutzgebiete zulassen.
\end{abstract}

\section{$1 \quad$ Einleitung und Grundlagen}

Erneuerbare Energien gewinnen seit Jahren immer mehr an Bedeutung und haben sich bereits als Zukunftsmarkt etabliert. Neben der Verringerung des $\mathrm{CO}_{2}$-Ausstoßes bietet der Wandel des Energiesystems Vorteile wie den Ausstieg aus der Kernenergie und die Eindämmung der Importe von fossilen Rohstoffen. Daraus resultierend soll die Deckung des Energiebedarfs bis 2050 zu 80 Prozent aus erneuerbaren Energien bestehen (BMWi, 2020).

Ein wichtiger Schritt in diese Richtung ist das Erneuerbare-Energien-Gesetz 2017 (EEG 2017). Neben Inhalten wie der Verbindung des Ausbaus der erneuerbaren Energien mit dem Netzausbau steht eine Veränderung der Förderrichtlinien von neuen Windkraftanlagen (WKA) im Zentrum des EEG 2017. 
Hier wurde ein Rückwärtsauktionssystem eingeführt, wo der Investor, welcher die geringste Einspeisevergütung fordert, die Genehmigung zum Bau und zum (geförderten) Betrieb erhält. Da durch dieses System die Wirtschaftlichkeit von erneuerbaren Energien sinkt, scheinen Gebiete mit geringeren Standorterträgen nicht mehr interessant. Um diesen Effekt auffangen zu können, wurde ein Korrekturfaktor für die Vergütung eingeführt. Dieser wird anhand des Standortertrags und Referenzertrags der geplanten Turbine und Nabenhöhe berechnet. Falls also der Standortertrag niedriger als der Referenzertrag ausfällt, liegt der Korrekturfaktor über 1, bei einem sehr guten Standort (also einem hohen Standortertrag) liegt er unter 1.

Um die ökonomische Fairness dieses Systems zu überprüfen, wurde eine Simulationsanalyse auf Basis der vom Deutschen Wetterdienst (DWD) bereitgestellten Windgeschwindigkeiten (vgl. DWD, 2014) durchgeführt. Die Ergebnisse dieser Analyse wurden mittels GIS-Anwendungen weiterverarbeitet und auf den gesamten deutschen Raum übertragen. Anders als bei bisherigen Einschätzungen des Windpotenzials lassen die hier entstandenen Daten nicht nur Auskünfte über den Ertrag einer beispielhaften Turbine (vgl. Umweltbundesamt, 2013), sondern auch über den Korrekturfaktor, den mit dem Korrekturfaktor gewichteten Ertrag sowie über die beste Turbinen-Höhen-Kombination in einem $200 \mathrm{~m} \mathrm{x}$ 200 m Raster zu.

Um die Daten und Ergebnisse besser zugänglich zu machen, wird eine Webapplikation entwickelt, welche als Tool zur Analyse von Standorten sowie als Visualisierung der Ergebnisse dient. Im Folgenden wird auf die Datenbasis und deren Aufbereitung sowie auf die Umsetzung der Visualisierung im Web eingegangen.

\section{Datenbasis und Aufbereitung}

Die Ergebnisse der Simulationsanalyse liegen in tabellarischer Form vor. Auf Basis der vom DWD ermittelten mittleren Windgeschwindigkeiten werden für jede Windgeschwindigkeit $(1,1 \mathrm{~m} / \mathrm{s}$ bis $8,4 \mathrm{~m} / \mathrm{s})$ der Ertrag und Korrekturfaktor für 84 Turbinen auf Nabenhöhen zwischen $70 \mathrm{~m}$ und $150 \mathrm{~m}$ berechnet. Zusätzlich beinhalten die Ergebnisse die „beste“ (d. h. vergütungsmaximale) TurbinenHöhen-Kombination inklusive ihrer Ertragswerte.

Um die gewonnenen Informationen visualisieren zu können, müssen zunächst Geometrien erstellt werden. Da sich die Ergebnisse allesamt auf Windgeschwindigkeiten beziehen, bietet sich hier der Ausgangsdatensatz, das DWD- 
Windgeschwindigkeitsraster, an. Dieser wird durch eine Polygonisierung in Vektordaten umgewandelt. Wie in Abbildung 1 gezeigt, werden bei diesem Verfahren benachbarte Zellen zu Polygonen zusammengeführt (vgl. Esri, 2020). Die Windgeschwindigkeit wird zusätzlich in der Attributtabelle hinterlegt und kann somit als Verknüpfungs-ID herangezogen werden. Dieses Verfahren wird für Darstellungen des Energieertrags, Korrekturfaktors und des gewichteten Ertrags durchgeführt.

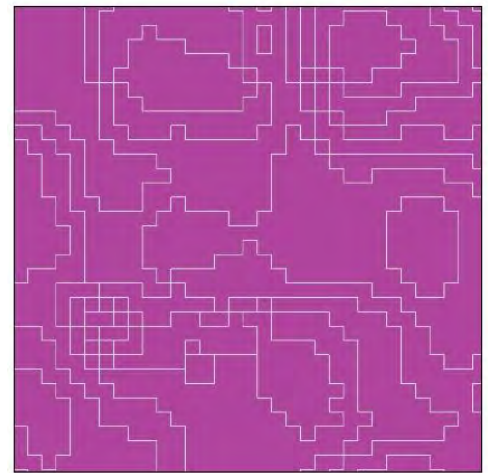

Vektordaten

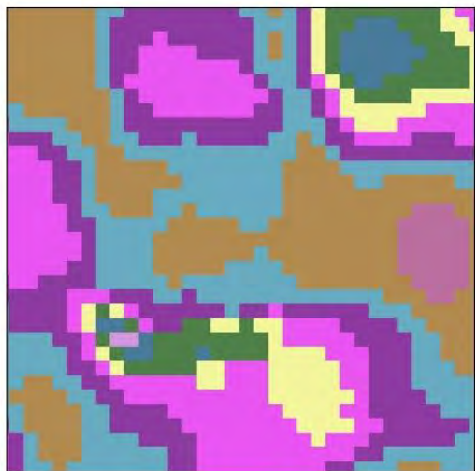

Rasterdaten

Abbildung 1: Polygonisierung der Rasterdaten

\section{Webapplikationsentwicklung}

Um die berechneten Daten öffentlich zugänglich zu machen, soll eine Webapplikation begleitend zur Forschungsreihe entstehen. Im Folgenden wird zunächst eine grobe Struktur und nachfolgend die Funktionsweise der Webapplikation erläutert.

\subsection{Struktur}

$\mathrm{Zu}$ Beginn wird eine erste grobe Weboberfläche (siehe dazu Abbildung 2) unter Einbezug der HTML-CSS-Bibliothek Bootstrap entwickelt. Ein besonderes Augenmerk wird hier auf die einfache Gestaltung gelegt, wodurch eine schnelle Übersicht gegeben ist. Elemente wie ein klassischer „Table of Contents“, ein Importbereich der Daten sowie die Nutzung von Tools werden durch die Javascript-Bibliothek jQuery funktionsfähig. 


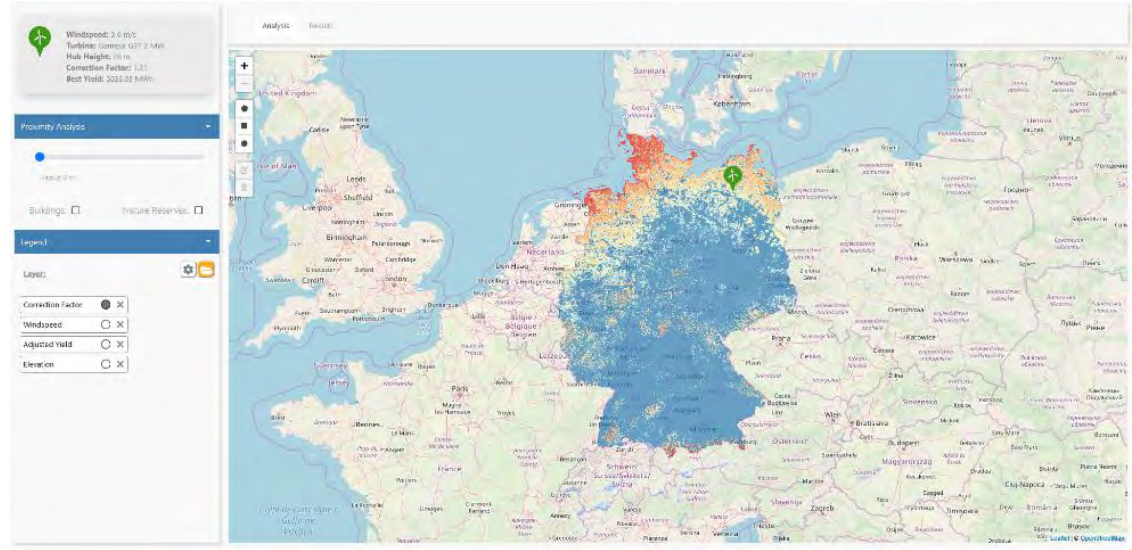

\section{Abbildung 2: Überblick über die Webapplikation}

Nachdem die Oberfläche in der Grundausrichtung fertiggestellt ist, wird die Webmap hinzugefügt. Hier fällt die Entscheidung auf die Bibliothek Leaflet, da sich diese im Vergleich zu OpenLayers auf relevante Funktionen beschränkt und dadurch wenig Speicherplatz benötigt. Weitere Funktionen können zudem als Plugin hinzugefügt werden.

Die Daten werden serverseitig mittels Geoserver und PostGIS-Datenbank verwaltet und angeboten. Auf dem Geoserver sind die Rasterdaten hinterlegt, wodurch ein Zugang mittels Web Map Service (WMS) ermöglicht wird. Die oben erwähnte Ergebnistabelle, Gebäudedaten von OpenStreetMap sowie Naturschutzgebiete sind auf der PostGIS-Datenbank gespeichert.

\subsection{Funktionsweise}

Die Funktionsweise der Webapplikation teilt sich in zwei Bereiche auf. Sie kann im klassischen Sinn als Viewer verwendet werden, in welchem die Forschungsergebnisse als Rasterdaten betrachtet werden können. Hierfür werden unterstützend ein Importbereich, ein Table of Contents inklusive Layerfunktionalität sowie Layereinstellungen wie Transparenz bereitgestellt. Angeboten werden derzeitig folgende Datensätze:

- Windgeschwindigkeit

- Windrichtungen

- Standortertrag der besten Turbinen-Höhen-Kombination

- Gewichteter Ertrag der besten Turbinen-Höhen-Kombination 
- Korrekturfaktor der besten Turbinen-Höhen-Kombination

- $\quad$ Naturschutzgebiete (Umweltbundesamt)

- Hangneigungen

- Digitales Geländemodell

Die weiterführende Funktionsweise stellt die Standortanalyse dar. Wie in Abbildung $3 \mathrm{zu}$ sehen, wird für einen gewählten Punkt die Windgeschwindigkeit ermittelt, wodurch ein Matching mit den Daten auf der PostGIS-Datenbank ermöglicht wird. Auf diesem Weg können Informationen zur besten Turbine und deren Nabenhöhe, Korrekturfaktor und dem Ertrag ausgegeben und angezeigt werden.

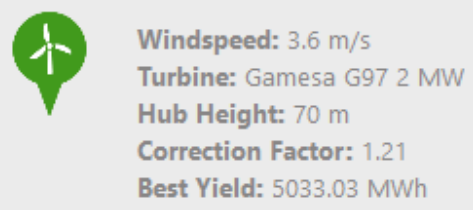

\section{Abbildung 3: Standortinformationen}

Diese Informationen bieten erste Hinweise auf die Eignung eines Gebietes. Zur weiteren Untersuchung wird der Analysebereich „Proximity Analysis“ angeboten (siehe Abbildung 4). Hier wird ein Tool zur interaktiven Erstellung eines Buffers implementiert, welcher eine Umkreisanalyse ermöglicht. 
Via Schieberegler kann der Umkreis aktiv größer oder kleiner gewählt werden. Implementiert wird dieses Tool mithilfe der Javascript-Bibliothek Turf. Sie bietet bekannte Grundfunktionalitäten eines GIS und ist schnell zu implementieren. Da die Berechnungen clientseitig ausgerichtet sind, wird kein weiterer Netzverkehr erzeugt. Ausgehend vom erstellten Buffer können Gebäude und Naturschutzgebiete im vorgegebenen Radius ermittelt werden (siehe Abbildung $5)$.

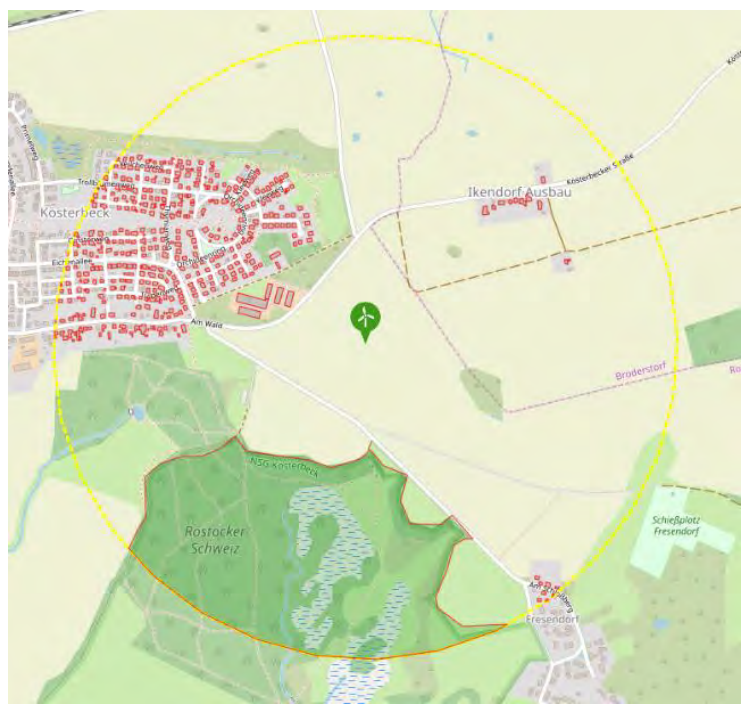

Abbildung 5: Umkreisanalyse - Ermittlung von Gebäuden und Naturschutzgebieten

Dazu wird die Bounding Box des Buffers ermittelt, mit deren Maßen eine Anfrage an den PostGIS-Server gesandt wird. Dieser sendet daraufhin alle Gebäude, welche sich innerhalb dieses Areals befinden, an den Client. Nun wird jedes empfangende Gebäude mit einem Intersect geprüft. Falls sich das Gebäude innerhalb des Buffers befindet bzw. falls es vom Buffer geschnitten wird, wird es in eine Feature Collection abgelegt und später auf der Webmap angezeigt. 


\section{$4 \quad$ Zusammenfassung und Ausblick}

Derzeitig ist die Webapplikation noch ein Prototyp und kann nur intern genutzt werden. Es ist geplant, sie so bald wie möglich live zu schalten. Sie soll ein Werkzeug zur ersten Untersuchung und Ermittlung von Windkraftpotenzialen sein. Es werden dem Nutzer nicht nur Potenziale anhand von Windgeschwindigkeiten dargestellt, sondern auch Informationen zur optimalen TurbinenHöhen-Kombination und den damit verbundenen Erträgen. Zusätzlich erlaubt die Einbindung von Geoprocessing-Werkzeugen eine interaktive Analyse des gewählten Standortes.

Zukünftig sollen weitere Werkzeuge implementiert sowie eine Ergebnisausgabe gestaltet werden. Auf diesem Weg wird einem Nutzer ein Gutachten über einen Standort ausgegeben, wodurch eine erste Einschätzung des Energiepotenzials und der Machbarkeit möglich gemacht wird.

\section{Literatur}

Bundesministerium für Wirtschaft und Energie (BMWi): Ziele - Windenergie auf See, https://www.erneuerbare-energien.de/EE/Navigation/DE/Technologien/

Windenergie-auf-See/Ziele/ziele.html, Zugriff am 20.02.2020.

DWD Climate Data Center (CDC): 200 m x 200 m Rasterdaten der mittleren jährlichen Windgeschwindigkeiten in $10 \mathrm{~m}$ bis $100 \mathrm{~m}$ Höhe (in 10-m-Stufen) und Weibullparameter für Deutschland, Version V0.1, 2014.

Esri: Raster in Polygon, https://pro.arcgis.com/de/pro-app/tool-reference/conversion/ raster-to-polygon.htm, Zugriff am 21.02.2020.

Umweltbundesamt: Potential der Windenergie an Land, Juni 2013. 

GIS-Technologie I 



\title{
Die Internetplattform Metroplaner - Das standardisierte Geoportal der Metropolregion Nordwest -
}

\author{
Robert Krätschmer
}

GDI-Service Rostock, Friedrichstraße 16, 18057 Rostock

robert.kraetschmer@gdi-service.de

\begin{abstract}
Interoperable Daten erlauben grenzüberschreitende, bruchstellenfreie Vergleiche, Aussagen und Analysen ohne hohe Datentransformationskosten. Für die Metropolregion Nordwest wurde deswegen die bestehende Plattform Metropolplaner unter Einbezug der Regionalen Raumordnungsprogramme der Landkreise und der Flächennutzungspläne der kreisfreien Städte der Region fortgeschrieben. Die Datenstrukturbasis des neuen Portals bilden der Datenaustauschstandard XPlanung für die deutsche Raumplanung sowie das Thema Bodennutzung von INSPIRE, der verpflichtende europäische Datenstandard. In zwei Projektschritten wurden die Daten in die beiden Standards konvertiert und daraufhin ein Portal aufgebaut sowie Dienste erstellt und eingebunden.
\end{abstract}

\section{$1 \quad$ Einleitung}

Eine Metropolregion als eng verflochtener Lebens- und Wirtschaftsraum profitiert von vielfältigen, auf unterschiedliche Planwerke zugreifenden Abstimmungen und Analysen. Um die Effizienz des damit verbundenen Datenaustausches zu optimieren, hat sich die Metropolregion Nordwest (Metropolregion BremenOldenburg im Nordwesten e. V.) entschlossen, ihr bereits existierendes Portal Metropolplaner auf Basis der standardisierten Datenmodelle XPlanung und INSPIRE in einem Pilotprojekt nachhaltig fortzuschreiben. Die Präsentation der Planungsinhalte für die elf zur Metropolregion gehörenden Landkreise und fünf kreisfreien Städte enthält neben der bereits im ursprünglichen Portal vorhandenen rasterbasierten Darstellung der Plankarten nun auch vektorielle, individuell zuschaltbare Planinhalte. Weiterhin werden relevante Plandokumente, Metadatenschnittstellen und Geowebdienste gleichfalls vom Portal aus erreicht. Als clientseitige Oberfläche wird das von der Freien und Hansestadt Hamburg ent- 
wickelte OpenSource Geoportal „Masterportal“ ${ }^{\text {“1 }}$ zusammen mit einer Landingpage verwendet.

\section{Der Metropolplaner}

Am Projekt Metropolplaner sind als Datenhalter die Landkreise Ammerland, Cloppenburg, Cuxhaven, Diepholz, Friesland, Oldenburg, Osnabrück, Osterholz, Vechta, Verden und Wesermarsch sowie die kreisfreien Städte Bremen, Bremerhaven, Delmenhorst, Oldenburg und Wilhelmshaven beteiligt. Während im Portal für die Landkreise die Regionalen Raumordnungsprogramme (RROP) der Regionalplanung dargestellt werden, werden für die kreisfreien Städte Flächennutzungspläne verwendet, um eine möglichst flächendeckende Abbildung der Planinhalte der Region zu bieten. Zusätzlich ist ein bestehender Dienst des Landesraumordnungsprogramms (LROP) Niedersachsens eingebunden. Flächennutzungsplandaten der Landkreise und Bebauungsplan- oder Landschaftsplandaten der Metropolregion sollen dagegen nicht dargestellt werden.

Das Projekt ist in zwei Schritten aufgebaut Ein erster Projektschritt beinhaltet die Transformation aller rechtsgültigen regionalen Raumordnungsprogramme nach XPlanung und INSPIRE. Da zwei Landkreise zum Zeitpunkt des Projekts keine rechtsgültigen Raumordnungspläne besitzen, wurden diese nicht transformiert. Weiterhin wurden in diesem Projektschritt Schulungen in den jeweiligen Landkreisen durchgeführt und Workshops für den Erfahrungsaustausch der kreisfreien Städte gehalten. So konnte neben einer Datenbasis gleichfalls Knowhow zur Erstellung und Verarbeitung der Datenformate vor Ort aufgebaut werden. Dieser Projektschritt wurde 2017 begonnen und 2018 fertiggestellt.

In einem zweiten, seit Anfang 2019 laufenden Projektschritt sollen das eigentliche Portal Metropolplaner sowie zugehörige Dienste erstellt werden. Für die Regionalen Raumordnungsprogramme wird auf die Daten des ersten Projektschritts zugegriffen. Die kreisfreien Städte verfügen dagegen über eigenständige Transformationsmöglichkeiten nach XPlanung. Die so erstellten und übermittelten GML-Daten können in eine existierende objektrelationale XPlanungDatenbank eingelesen werden und gleichfalls ausgegeben werden. In weiteren Workshops während des zweiten Projektschrittes sind neben einer regelmäßigen Präsentation des Projektstandes auch offene Fragen, zum Beispiel zu Datenli-

${ }^{1}$ https://www.hamburg.de/geowerkstatt/8856396/masterportal/ 
zenzen, Vektor-Attribuierung, der Möglichkeit der Darstellung von Entwurfsständen und der Fortführung der Plandaten besprochen worden. Das Portal selbst wird voraussichtlich im Sommer 2020 nach Abschluss des zweiten Projektschrittes und eingehender Testphase freigeschaltet.

\section{XPlanung und INSPIRE}

Um den verlustfreien Austausch der Planinformationen zwischen den Planungsträgern effizient zu gestalten, wird XPlanung als Format der zugrunde liegenden Daten und Dienste verwendet. Der Standard ,ist die Norm für die Struktur, den Inhalt und die Form von Daten/Informationen zur Bereitstellung von räumlichen Planwerken“ (Leitstelle XPlanung / XBau, 2018, S. 6).

Während die kreisfreien Städte ihre Daten eigenständig nach XPlanung transformieren, sind für die Raumordnung alle rechtsgültigen regionalen Raumordnungsprogramme der Landkreise über die Software XPlankonverter des Bundesinstituts für Bau-, Stadt- und Raumforschung (BBSR) im Bundesamt für Bauwesen und Raumordnung (BBR) nach XPlan-GML und INSPIRE-GML transformiert worden. Als Plugin der WebGIS-Software kvwmap erlaubt der Konverter die Transformation von Eingangsdaten (meist im Shape-Format) über die Erstellung von SQL-basierten Regeln und die Erfassung von planbeschreibenden Daten über Formulare. Eine Kopie der zugrunde liegenden PostGIS-Datenbank wird als Quelle für folgende flache MapServer-Gebrauchsdienste verwendet.

Bauleitplan- und Raumordnungsdaten werden in INSPIRE im Anwendungsschema „Geplante Bodennutzung“ des Themas „Bodennutzung“ im Anhang III abgebildet. Die Transformation hierzu wurde auf Basis der von der XLeitstelle in Hamburg bereitgestellten Transformationsregeln von XPlanung nach INSPIRE aufgebaut. ${ }^{2}$ Die Transformation von XPlanGML nach INSPIRE-GML findet über eine Extensible Stylesheet Language Transformation (XSL-T) statt. Die so erstellten INSPIRE-GML-Daten sind über einen GDAL ogr2ogrEinlesevorgang mit dem Applikationsschema-Treiber GMLAS wieder in eine aus dem XML-Schema erstellte Datenbank überführt worden. Diese dient als Datengrundlage der INSPIRE-Dienste.

${ }^{2}$ http://www.xplanungwiki.de/index.php?title=INSPIRE_Transformation 
Da einige Planträger bereits eigene Lösungen zur Erfüllung der INSPIREPflichten entwickelt oder geplant haben, werden für diese keine Dienste im Rahmen des Metropolplaner-Projekts erstellt. Existierende Dienste können aber als Layer in die Oberfläche des Portals eingebunden werden.

\section{$4 \quad$ Dienste}

Um die Handhabbarkeit in gängiger GIS-Software zu gewährleisten, wurden auf Basis der konvertierten Daten flache XPlanung-Gebrauchsdienste mit MapServer erstellt. Hierfür wurden die überlieferten XPlanGML-Daten der Flächennutzungspläne der kreisfreien Städte gleichfalls in die bereits mit den RROP-Daten befüllte, vorhandene XPlanung-Datenbank gelesen.

Die einheitliche Darstellung der RROP-Planzeichen wurde aus den Vorgaben der niedersächsischen Arbeitshilfe „Planzeichen in der Regionalplanung“3 übernommen und in Abstimmungsprozessen mit den Planträgern teilweise angepasst, zum Beispiel falls ältere Planwerke nicht mehr adäquat über die Darstellungen der neueren Handreichung abgebildet werden konnten. Die Darstellung der Flächennutzungspläne wurde in Abstimmung mit den Planträgern anhand der vorhandenen Planwerke und in Anlehnung an die Planzeichenverordnung (PlanVZ 90) aufgebaut.

Zur Erfüllung der verpflichtenden Vorgaben von INSPIRE an Dienste wurde die Software GeoServer mit den Erweiterungen AppSchema zur Erstellung von schemakonformen Diensten und der INSPIRE-Erweiterung zur Befüllung von zusätzlich geforderten Dienst-Metadaten verwendet. Die von INSPIRE geforderten Metadaten zu Layern und Daten werden in den jeweiligen Metainformationssystemen der einzelnen Planträger gehalten und über die vorgeschriebene Daten-Dienste-Kopplung in GeoServer verlinkt. Die Darstellung der WMSDienste verwendet die von den Datenspezifikationen des Themas „Bodennutzung" vorgeschriebenen Darstellungsregeln.

${ }^{3} \mathrm{http}: / /$ www.nlt.de/staticsite/staticsite.php?menuid=80\&topmenu=64 


\section{Das Masterportal Hamburg}

Als Geoportal des Metropolplaners wurde das Masterportal Hamburg verwendet. Es baut unter anderem auf OpenLayers, BackboneJS, Bootstrap und jQuery auf. Als rein clientseitige Software erlaubt es das Einbinden von OGCkonformen Dienstschnittstellen, deren Layer- und Einstellungskonfiguration über JSON-Dateien stattfinden, die zur Laufzeit gelesen werden. Die Oberfläche ist responsiv gestaltet, sodass neben Desktop- auch Mobil- oder TabletDarstellungen unterstützt werden.

Eine zusätzlich zum Portal erstellte Landingpage enthält weiterhin Informationen zum Projekt, relevante Plandokumente, GML-Dokumente der konvertierten Pläne sowie Informationen zu Nutzungsbedingungen und Datenschutz.

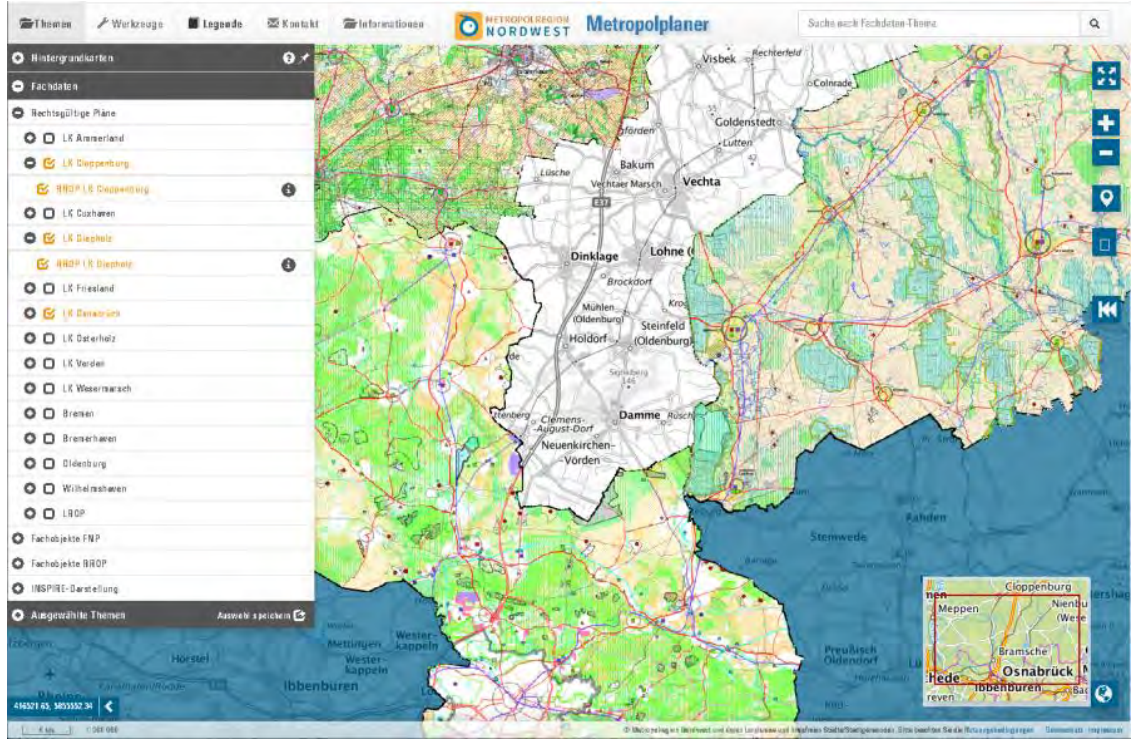

Abbildung 1: Darstellung der Metropolplaner-Oberfläche mit dargestellten Rasterinformationen

\section{$6 \quad$ Fazit}

Zusammenfassend bietet der neu entwickelte Metropolplaner die Möglichkeit zur interoperablen Darstellung und Nutzung von Planinhalten der Metropol- 
region. Aufgrund der Trennung von Diensten, Oberfläche und Metadaten, der Möglichkeit zur Einbindung von externen standardisierten Daten und Diensten sowie der konsequenten Verwendung von quelloffenen Werkzeugen kann der neue Metropolplaner nachhaltig genutzt, gewartet und ergänzt werden.

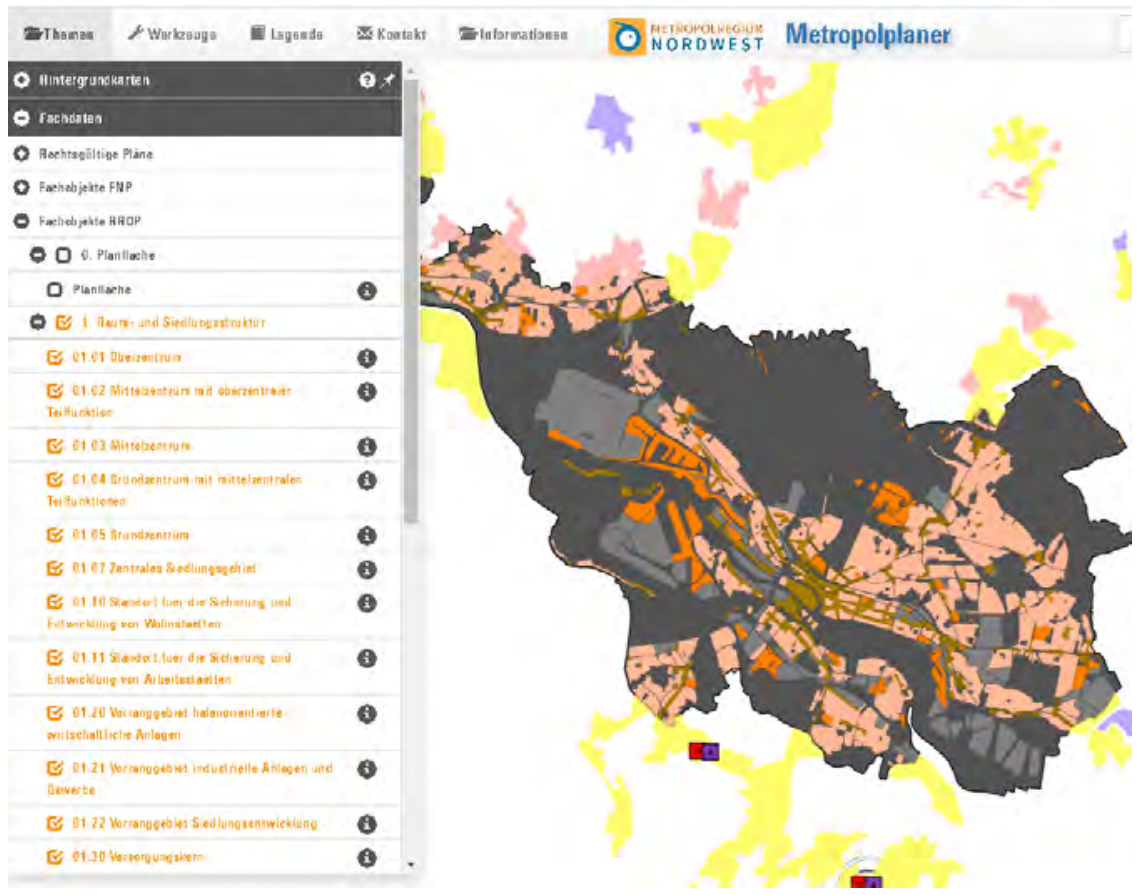

Abbildung 2: Darstellung von ausgewählten Flächennutzungsplandaten zur baulichen Nutzung Bremens sowie Raum- und Siedlungsstrukturdaten der umgrenzenden Regionalen Raumordnungsprogramme

\section{Literatur}

Leitstelle XPlanung / XBau: Handreichung XPlanung Xbau, Stand Oktober 2018. Online: erreichbar unter:

http://www.xleitstelle.de/sites/default/files/2019-03/Handreichung_XPlanungXBau_1.pdf 


\title{
Embedded GIS - die einfache Bereitstellung von Geodaten-Funktionalität in sachdatenorientierten Fachanwendungen mithilfe der Low-Code- Technologie
}

\author{
Robert Beyer \\ Scopeland Technology GmbH \\ Düsterhauptstraße 39-40, 13469 Berlin \\ rbeyer@scopeland.de
}

\begin{abstract}
Dieser Beitrag beschreibt die Entwicklung der web-basierten Fachanwendung MARLIN des Bundesamtes für Seeschifffahrt und Hydrographie zur Erfassung, Verwaltung und Auswertung von Daten im Rahmen der Planung und Realisierung von Offshore-Windparks mithilfe der Low-Code-Plattform SCOPELAND.
\end{abstract}

\section{$1 \quad$ Einleitung}

Da Geodaten immer mehr an Bedeutung gewinnen, bleibt es nicht aus, dass Fachabteilungen von Unternehmen und Behörden zunehmend auch anspruchsvolle Geodaten-Funktionalität und Kartendarstellungen als integralen Teil ihrer ansonsten eher alphanumerischen Businessanwendungen erwarten - etwa für eine bequeme geographische Auswahl von Kunden oder Antragstellern, für automatische Verortungsservices oder für komplexe Geodaten-Berechnungen. Aktuell gibt es immer mehr Anwendungsfälle, bei denen Geodaten- und Sachdatenfunktionalität gänzlich miteinander verschmelzen.

Bislang waren sämtliche Geodaten-Features nur den richtigen GI-Systemen vorbehalten, die noch eher auf die Nutzung durch GIS-Spezialisten als durch sonstige Sachbearbeiter ausgelegt sind. Eine Individualprogrammierung solcher Geodaten-Features in Fachanwendungen wäre viel zu aufwendig, und auch die Integration von Komponenten eines GI-Systems in normale Datenbankanwendungen ist vergleichsweise aufwendig und immer problembehaftet. Deshalb sind alternative Wege und Ansätze gefragt, mit denen man als normaler Anwendungsentwickler mit geringem Aufwand und ohne spezielles GIS-Know- 
how relativ anspruchsvolle Geofunktionalität in Businessanwendungen einbauen kann.

Die einfache Bereitstellung von Geodaten-Funktionalität in sachdatenorientierten Fachanwendungen auf Basis der Low-Code-Technologie wird bei Scopeland Technology als Embedded-GIS-Ansatz bezeichnet. Dieser Ansatz basiert auf der Low-Code-Technologie und wurde beim Bundesamt für Seeschifffahrt und Hydrographie zur Erfassung, Verwaltung und Auswertung von Daten im Rahmen der Planung und Realisierung von Offshore-Windparks angewendet. Realisiert wurde die web-basierte Fachanwendung MARLIN in der Low-CodePlattform SCOPELAND. Sie kann als digitaler Inkubator für fortgeschrittene Bewertungen der Meeresumwelt verstanden werden.

\section{Die Low-Code-Technologie}

Die Low-Code-Technologie basiert auf dem Grundgedanken, dass sich typische Businessanwendungen aus immer denselben technischen Grundfunktionen zusammensetzen lassen und sie folglich interaktiv am Bildschirm per Konfiguration anstelle von Programmierung zusammengesetzt werden können. Anstelle klassischer Individualprogramme kommt im Grunde genommen nur vorgefertigter Programmcode mit einem festen Set an Features zum Einsatz. Angesichts der brachialen Effekte der Low-Code-Technologie prophezeien die führenden US-Analysten Forrester Research und Gartner, dass künftig ein Großteil sämtlicher Business-Anwendungen mit Low-Code-Methoden entwickelt werden wird, und das schließt auch Business-Anwendungen mit Geodaten-Features mit ein.

Dieses Konzept, das allen Low-Code-Plattformen gemeinsam ist, legt den Gedanken nahe, eine Vielzahl an zusätzlichen Features out-of-the-box mitzuliefern, die zwar nur in dem einen oder anderen Anwendungsfall benötigt werden, dort aber sehr effektiv helfen, immense Mehraufwände und Extrakosten zu vermeiden. Kein Wunder, dass auch integrierte Kartendarstellungen und SpatialFunktionen Eingang in die Low-Code-Technologie gefunden haben.

Auf die eigenständige Erzeugung von Basemaps und andere Kernfunktionen der großen GI-Systeme wird ganz bewusst verzichtet. Stattdessen beschränkt man sich auf die Verwendung allgemein verfügbarer, kommerzieller und nichtkommerzieller Kartenservices, wie etwa von Esri, Google, Open Street Map oder das amtliche BKG-Kartenmaterial. Auf diese Basemaps werden nun von der Low-Code-Plattform die aktiven GIS-Objekte der Anwendung, manchmal zusammen mit Geodaten aus sonstigen verfügbaren Services, aufgeblendet. Der 
Effekt ist so beeindruckend, dass zunehmend auch für andere, ganz normale Fachanwendungen Geodaten-Erweiterungen nachgefragt werden.

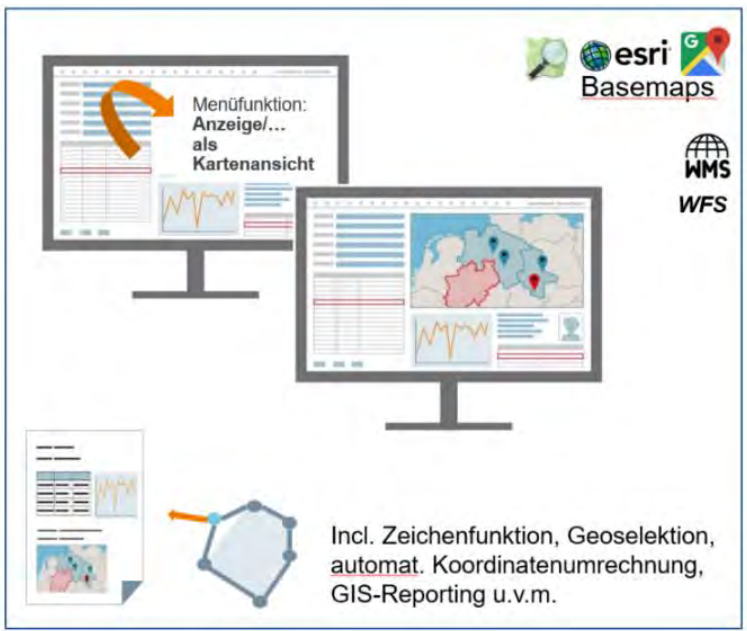

Abbildung 1: Der Embedded-GIS-Ansatz: Einbindung von Kartendarstellung und Geodatenverarbeitung mit einem Mausklick

\section{Die web-basierte Fachanwendung MARLIN des Bundes- amtes für Seeschifffahrt und Hydrographie}

Das Bundesamt für Seeschifffahrt und Hydrographie (BSH) ist eine Bundesbehörde, die sich als maritimer Dienstleister des Bundes versteht. Es ist dem Bundesministerium für Verkehr und digitale Infrastruktur zugeordnet. Die Aufgaben des BSH gliedern sich in die Bereiche Meereskunde, Nautische Hydrographie, Schifffahrtsförderung und zentrale Aufgaben.

Gefördert durch das Bundesministerium für Wirtschaft und Energie (BMWI) (FKZ 0325921) wird derzeit am Bundesamt für Seeschifffahrt und Hydrographie (BSH) in Zusammenarbeit mit dem FTZ der Universität Kiel und dem Alfred-Wegener-Institut (AWI) die Fachanwendung MARLIN entwickelt: ein web-basiertes Informationsnetzwerk für alle meeresbiologischen Meeresdaten aus der Umweltverträglichkeitsprüfung (UVP) und der Überwachung von Offshore-Windpark- und Netz-Projekten. 
Im Zuge der Planung und Realisierung von Offshore-Windparks in den deutschen Hoheitsgewässern der Nord- und Ostsee erfassen die Antragsteller, und später die Betreiber der Anlagen, biologische Daten zu den Schutzgütern Benthos, Fische, Meeressäuger und Avifauna. Dies erfolgt im Rahmen der geforderten Umweltverträglichkeitsstudien (UVS) und des begleitenden Monitorings gemäß dem BSH-Standarduntersuchungskonzept (StUK). Die Zusammenführung und Erfassung der Daten mithilfe individualisierter Excel-Importe und der damit notwendigen Prüfungen auf Gesamtheit und Plausibilität sowie die Analyse und Präsentation der verarbeiteten Daten werden aktuell durch das Fachinformationssystems MARLIN des BSH in Zusammenarbeit mit der LowCode-Plattform SCOPELAND realisiert.

In diesem Fachinformationssystem stehen räumliche Analysen über die Präsentation der Daten in Kartenanwendungen und dem Export der Ergebnisse in Geodaten-Formaten zur Verfügung. Ebenso ist die Einbindung von externen und internen Geodaten integraler Bestandteil der Anwendung.

\section{Anwendungsentwicklung mit der Low-Code-Plattform SCOPELAND}

In der Fachanwendung MARLIN werden Geodaten in mehreren Phasen der Datenauswertung genutzt. So werden importierte Daten hinsichtlich ihrer räumlichen Plausibilität geprüft, zum Beispiel, ob die erhobenen Koordinaten zu der definierten Projektfläche passen. SCOPELAND unterstützt die Entwicklung dieser Prüfungen, indem diese räumliche Abfrage als Möglichkeit der Datenselektion zur Verfügung gestellt wird und durch den Anwendungsentwickler nicht programmiert werden muss.

Die Erstellung der Kartenauswertungen und Kartenexporte durch den Anwendungsentwickler wird durch eine Vielzahl an Funktionen durch die Low-CodeTechnologie vereinfacht und der Aufwand an Programmierarbeiten wird deutlich verringert.

In der Fachanwendung MARLIN werden so mit wenig Programmieraufwand Geodatenprodukte wie Stations-, Dichte- oder Verteilungskarten realisiert. Die Erstellung der Produkte wird durch die Möglichkeiten der freien Selektion nach Themen oder Katalogen sowie der räumlichen Suche nach definierten Gebieten wie der deutschen Ausschließlichen Wirtschaftszone (AWZ) oder Flächen der Windparks durch den Endanwender begleitet. Eine Administrationsoberfläche ermöglicht es, in der Fachwendung externe Geodaten als Datei, WFS oder 
WMS für die Darstellung und Selektionsmöglichkeit in den Kartenanwendungen zu definieren.

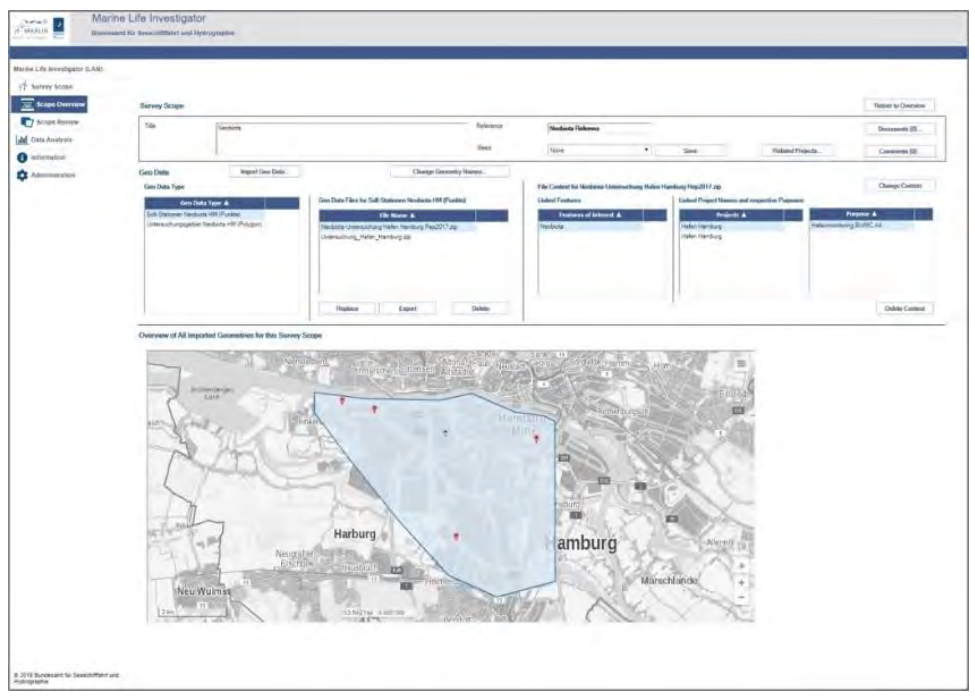

Abbildung 2: Die kombinierte Sachdaten- und Geodatenanwendung MARLIN

\section{Besonderheiten von MARLIN}

Großflächige und hochauflösende integrierte meeresökologische Informationssysteme kombinieren verschiedene ökologische und umweltbezogene Daten in Raum und Zeit, wie beispielsweise Biodiversität und trophische Beziehungen. Dieser ganzheitliche Ansatz ermöglicht neue Richtungen für die Analyse und Modellierung ökologischer Muster und Prozesse und schafft eine Plattform für die Entwicklung und Anwendung fortschrittlicher Instrumente für das Management und die Regulierung der Meere.

Das MARLIN-Netz verbindet nach Fertigstellung langfristige, qualitätsgeprüfte raumbezogene biologische Daten aus der Umweltverträglichkeitsprüfung und dem Monitoring aller deutschen Offshore-Windpark- und Netzprojekte. Es umfasst Karten zur Artenverteilung, Abundanz- und Biomasseinformationen sowie Messungen der Biodiversität in Zeit und Raum. 


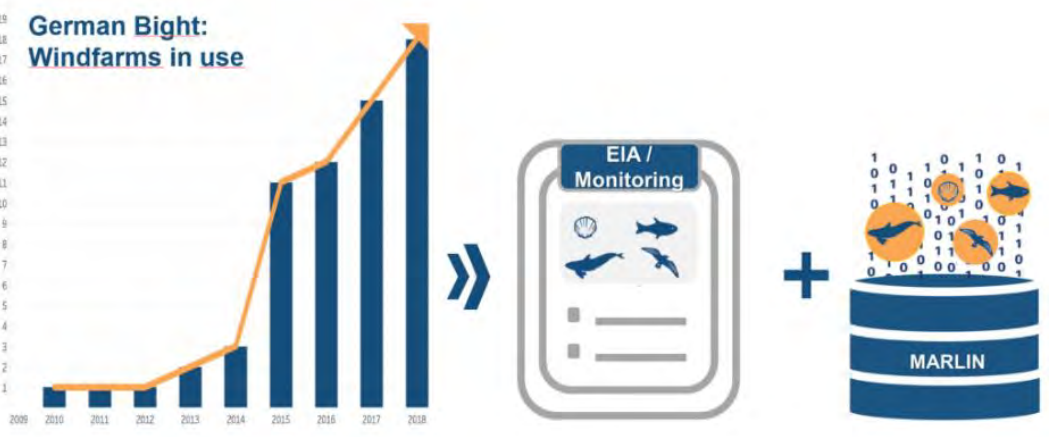

\section{Abbildung 3: Umweltverträglichkeitsprüfung / Data Monitoring von Offshore- Windparks}

Die aggregierten Produkte werden als Web Services für die Öffentlichkeit veröffentlicht. Darüber hinaus bietet MARLIN intuitive Auswahlwerkzeuge für die individuelle Datenauswahl oder maßgeschneiderte ProduktgenerierungsWorkflows (Use Cases) und komfortable Daten-Download-Funktionalitäten für autorisierte Nutzer.

MARLIN kann als ein leistungsfähiges Werkzeug für Managementzwecke im Zusammenhang mit der Bewertung von Regulierungsfragen wie der zeitlichen und räumlichen Variabilität der Artenvielfalt, der Abundanz und der Biomasse angesehen werden. Solche großflächigen und hochauflösenden Informationssysteme stellen eine solide wissenschaftliche Grundlage für verbesserte Überwachungsbewertungen und letztlich für ein nachhaltiges Ökosystemmanagement dar.

\section{Zusammenfassung}

Die Entwicklung der Fachanwendung MARLIN über den Import der erhobenen Daten, der Prüfung auf Plausibilität bis hin zur Erstellung der auf den Daten basierenden Produkte zeigt die Notwendigkeit einer Entwicklung, die es den Fachanwendern und den Anwendungsentwicklern ermöglicht, Anpassungen am System mit geringem Zeit- und Entwicklungsaufwand durchführen zu können. Dies betrifft auch die Möglichkeit, räumliche Abfragen zu erstellen oder Kartenprodukte zu verändern. 
MARLIN wird im Jahr 2020 online gehen und trägt zur Entwicklung eines langfristigen, qualitätsgeprüften Informationsnetzes für biologische Daten bei. Hiermit können künftig maßgeschneiderte Webanwendungen zum Laden, Austauschen und Analysieren biologischer Daten für verschiedene Benutzergruppen erstellt werden. Es wird eine Grundlage für die Modellierung von Meeresökosystemen und die Bewertung von kumulativen Auswirkungen geschaffen, und für zukünftige Monitoring-Untersuchungen können durch Neubewertung der aktuellen Erhebungsdesignkonzepte nach dem BSH-Standard Kostensenkungspotenziale evaluiert werden.

Auch bei diesem Projekt zeigt sich, dass die Low-Code-Technologie für die professionelle und anspruchsvolle Anwendungsentwicklung bestens geeignet ist und dass der Embedded-GIS-Ansatz durch die Integration von Kartendiensten und -darstellungen, geo-bezogenen Funktionen und sogar von umfassender GISFunktionalität in sachdatenbezogenen Datenbankanwendungen den Sachbearbeitern der Bundesbehörde die Arbeit mit MARLIN angenehmer gestaltet.

\section{Literatur}

Rudolf, H. (2018): Umweltdatenmanagement - Eine GEO-Inspiration. Bernhard Harzer www.bsh.de Verlag GmbH, 2018

https://epic.awi.de/id/eprint/48140 



\section{Landmanagement und Daseinsvorsorge}





\title{
Nutzung von Geodaten für das Landmanagement - Modellierung der Lagequalität aus freien Geodaten
}

\author{
Winrich Voß, Keno Bakker \\ Geodätisches Institut, Leibniz Universität Hannover \\ voss@gih.uni-hannover.de, bakker@infra-hannover.de
}

\begin{abstract}
Auf der Grundlage der INSPIRE-Richtlinie hat sich die flächendeckende Versorgung mit Geodaten deutlich beschleunigt und verbessert. Auf Basis der positiveren Datenlage wird in dem vorgestellten Projekt ein Modell entwickelt, das mittels Geodaten die „Standortqualität" im Sinne der immobilienmarktrelevanten "Lage“ modelliert. Der Beitrag stellt in Grundzügen den aktuellen Stand der Modellentwicklung dar. Der Praxisbedarf eines solchen Modells ist hoch, da die Anzahl und Verfügbarkeit von direkten Immobilienmarktdaten (Verkaufsfällen) vielfach unzureichend ist. Das entwickelte Modell soll insbesondere für kaufpreisarme Lagen die Immobilienwertermittlung und Marktanalyse mit zusätzlichen Informationen unterstützen, z. B. bei der Bodenrichtwertermittlung. Damit trägt es zur Verbesserung der Transparenz am Grundstücks- und Immobilienmarkt bei, einem vorrangigen Ziel der Arbeit der Gutachterausschüsse in Deutschland.
\end{abstract}

\section{$1 \quad$ Einleitung}

Für das Landmanagement sind Einschätzungen zur Lagequalität eines Standortes von grundlegender Bedeutung. Entsprechende Informationen sind z. B. im Rahmen von Projektentwicklungen oder für die Grundstückswertermittlung unverzichtbar. Sehr kleinteilig beschäftigt sich die ImmobilienProjektentwicklung und -bewertung mit den Standortfaktoren in Form der „Lage einer Immobilie“. Die traditionellen Standorttheorien unterscheiden die Faktoren der Makro- und der Mikroebene sowie in harte und weiche Standortfaktoren, wobei Letztere zunehmend vielfältige Ausprägungen erfahren. Die Bedeutung der Standorteigenschaften wird nicht zuletzt durch das Credo verdeutlicht, dass nur „die Lage, die Lage und nochmals die Lage“ ausschlaggebend sei. Eine besondere Herausforderung stellt die Differenzierung der unterschiedlichen 
„Lagen“ auf dem Immobilienmarkt sowie ihre Einschätzung durch die individuellen Präferenzen der Kaufbeteiligten dar.

Grundlage der Lageeinschätzung bildet die Analyse der wertrelevanten Lagemerkmale (auch Einflussfaktoren oder Indikatoren). Leider stehen diese Primärdaten in vielen räumlichen und sachlichen Teilmärkten nicht in ausreichender Anzahl und Qualität zur Verfügung (z. B. Innenstadtlagen oder ländliche Räume, Mehrfamilienhäuser oder Gewerbeimmobilien). So limitiert bei der Suche nach Vergleichsobjekten - z. B. im Rahmen einer Bewertung auf Basis des Vergleichswertverfahrens ( $§ 15$ ImmoWertV) - die Lage die Auswahl i. d. R. auf Fälle aus der Umgebung des Bewertungsfalls (vergleichbare Lage).

Vor dem Hintergrund dieser Anforderungen aus der Grundstückswertermittlung wird in diesem Beitrag ein Modell vorgestellt, dass „die Lage“ mithilfe der zunehmend verfügbaren Geodaten beschreibt und bewertet. Im Gegensatz zur Knappheit an unmittelbaren Marktinformationen stehen zunehmend mehr Geodaten frei zugänglich und regelmäßig aktualisiert zur Verfügung (Basis INSPIRE-Richtlinie). Soweit Immobilienbezug gegeben ist, besteht das Ziel, die Informationen aus Geodaten ergänzend für die Marktanalyse und Wertermittlung von Immobilien nutzbar zu machen.

Neu gegenüber herkömmlichen Standortanalysen ist dabei der für die Lageerfassung erforderliche kleinräumige Ansatz, bestehend aus einem Set von Makro- und Mikrolage-Indikatoren, sowie deren Gewichtung und Wertung für unterschiedliche Teile des Immobilienmarktes. Das Modell nutzt GIS-Analysen in Kombination mit nutzwertanalytischen Ansätzen. Es wird erwartet, dass landesweite Relationen von Lagequalitäten aufgestellt werden können, die Vergleiche erleichtern und die spärlichen Primärdaten aus Immobilienmarkttransaktionen über Regionen hinweg nutzbar machen können.

Der Beitrag erläutert die Forschungen am Geodätischen Institut Hannover in Zusammenarbeit mit den Niedersächsischen Gutachterausschüssen zur Nutzbarmachung von Geodaten als Ergänzung für fehlende Marktinformationen. Die bisherigen Arbeiten erfolgten im Rahmen des Forschungsprojektes „Interoperabilität von Geodaten am Beispiel aktueller Aufgaben der Wertermittlung“" (Bearbeitung M.Sc. Keno Bakker) mit Unterstützung und Finanzierung des Nds. Ministeriums für Inneres und Sport. Die anschließenden Darstellungen sind im Wesentlichen dem Beitrag „Voß/Bakker 2017, IÖR Schriften Band 73, Rhombus-Verlag Berlin, S. 113-122“ entnommen. 


\section{Projektansatz: Lagequalität aus Geodaten}

Durch ihren Raumbezug enthalten die Geodaten vielfältige Informationen über einen Standort bzw. über lagetypische Qualitäten eines Standortes. Ziel des Projektes ist die Schaffung einer zusätzlichen Datengrundlage aus raumrelevten Geodaten, die u. a. ergänzend in der Bodenrichtwertermittlung verwendet werden kann. Die aus Geodaten abgeleiteten differenzierten „Standortqualitäten“ sollen geeignet sein, die Wertigkeit einer Lage relativ zu Nachbarlagen und zu vergleichbaren Lagen andernorts abzubilden. Das Projekt legt den Fokus auf das Bundesland Niedersachsen.

Da die „Lage“ nicht durch ein einziges Geodatum zu erfassen ist, bedarf es eines aggregierenden Modells, das den Beitrag jedes verwendeten Geodatums zur Standortqualität insgesamt bewertet. Zudem ist zu berücksichtigen, dass die immobilienrelevante „Lage“ teilmarktabhängig ist; so ist eine gute Wohnlage i. d. R. nicht zugleich eine gute Gewerbelage. Die Verschneidung einer Vielzahl von Geodaten zu sozio-ökonomischen Gegebenheiten und Zusammenhängen bildet die wesentliche erklärbare Basis für den Wert einer Lage. Der Ansatz orientiert sich an der Standortanalyse und erfasst somit die Standortfaktoren (im Folgenden Indikatoren genannt) als einen wichtigen Teil der preisbildenden Faktoren einer Immobilie.

\section{$3 \quad$ Eckpunkte der Modellentwicklung}

Für die Modellbildung müssen einerseits die regionale bzw. überörtliche Einordnung (Makrolage) und andererseits die kleinteiligen Differenzierungen bis zur Quartiers- bzw. Baublockebene (Mikrolage) abgebildet werden. Diese Unterscheidung wird in Anlehnung an die Standortanalyse vorgenommen (Ottmann \& Lifka 2010). Makro- und Mikrolage werden durch unterschiedliche Indikatoren und Geodaten beschrieben. Das Modell ermittelt für beide Ebenen getrennte Teilbeträge, die schließlich zusammenzuziehen sind.

Für die Modellbildung sind verschiedene Entwicklungsschritte erforderlich, die jeweils für die Makro- und die Mikrolage zu durchlaufen sind:

1. Auswahl der möglichen Standortindikatoren pro Teilmarkt (Literaturrecherche und Experteneinschätzung). Auf dieser Basis wurde ein umfassender Katalog mit 80 lagerelevanten Indikatoren zusammengestellt, aus dem durch Expertenbefragungen im Rahmen einer Prioritätenanalyse die wichtigsten Indikatoren für die Makro- wie für die Mikrolage 
extrahiert wurden. Im Ergebnis sind je 15 Indikatoren mehrheitlich von den Experten als besonders relevant benannt worden.

2. Zuordnung von frei verfügbaren Geodaten zu den gewählten Standortindikatoren (Empirie). Im nächsten Schritt werden den Indikatoren geeignete Geodaten zugeordnet, sodass die Erfassung der Indikatoren aus frei verfügbaren und aktualisierten Geodaten gewährleistet werden kann. Dazu bedarf es teilweise der Kombination mehrerer Geodaten, um den gewünschten Indikator inhaltlich abzubilden (z. B. Bevölkerungsstruktur, Nähe zur Natur). Entsprechende Verschneidungen, insbesondere bei den entfernungsabhängigen Mikrolage-Indikatoren, erfolgen GIS-basiert (vgl. grundlegend Bill 2016).

3. Gewichtung der einzelnen Indikatoren mittels Experteneinschätzungen im Rahmen einer Prioritätenanalyse. Zweifelsohne sind die 30 Indikatoren nicht alle von gleicher Bedeutung für die Lagequalität. Eine möglichst objektive Gewichtung kann über eine breit gefächerte Stichprobe an Prioritätenanalysen erreicht werden. Hierfür wurden im ersten Schritt 11 Experten des Gutachterausschusswesens in Niedersachsen befragt, im Weiteren verifiziert durch eine bundesweite Befragung von Wertermittlungssachverständigen.

4. Bewertung der Indikatoren für den jeweiligen Standort bzw. das jeweilige Rasterelement (Zielerreichungsgrad mittels GIS-Analysen). Eine Bewertung der Indikatoren erfolgt differenziert nach ihrer räumlichen Zuordnung zur Mikro- oder Makroebene. Die Indikatoren der Makroebene setzen sich aus statistischen Kennzahlen zusammen, die der Mikroebene überwiegend aus geometrischen Merkmalen. Basis sind die Bewertungen mit den Ziffern 1, 2 und 3. Dabei steht die Ziffer 3 für die bestmögliche Bewertung eines Indikators.

5. Berechnung der Standortqualität bzw. des Lagewertes als Makro- und als Mikrolagequalität erfolgt mit einem nutzwertanalytischen Ansatz (Fürst \& Scholles 2008). Sind die Gewichtung mithilfe der Prioritätenanalyse und die o. g. Punktebewertung erfolgt, können durch deren Multiplikation die Teilwerte errechnet werden. Die Summierung der Teilwerte wiederum ergibt den Gesamtwert (= Nutzwert als Ergebnis der Nutzwertanalyse) für Makro- bzw. Mikrolage, die die Standortqualität im jeweils betrachteten Immobilienteilmarkt repräsentieren. Je höher diese Werte, desto höher die Qualität des jeweiligen Standortes. Das finale Produkt des Modells ist eine gewichtete Kombination aus den berechneten Standortqualitäten der Makro- und der Mikroebene. Dabei wird der Qualität der Mikrolage - aufgrund der üblichen Funktionsweise des Immobilienmarktes als überwiegend örtlich determiniert 
und vorbehaltlich der abschließenden Validierungsergebnisse - der klar höhere Gewichtsanteil zugesprochen.

Nur ein Modell, das alle relevanten Informationen aus Geodaten bewertend miteinander vereint, kann bezogen auf den jeweiligen Immobilienteilmarkt eine Aussage zur Qualität eines Standortes liefern. Die Standortqualität wird in dem hier beschriebenen Modell in einem relativen und einheitslosen Wert angegeben. Das Modell zur Berechnung der Lagequalitäten wurde flächendeckend für Niedersachsen mit einer räumlichen Auflösung von 1 x $1 \mathrm{~km}$ erstellt. In Gebieten mit hoher Siedlungsdichte erfolgt eine Verdichtung auf $500 \times 500 \mathrm{~m}$ Zellgröße (vgl. Abb. 1).

\section{$4 \quad$ Bisherige Ergebnisse}

Als wichtiges Ergebnis des Forschungsprojektes konnte gezeigt werden, dass die Darstellung einer differenzierten Standortqualität für Niedersachsen durch eine nach Immobilienteilmärkten differenzierte, nutzwertanalytische Aggregation von Geodatenbeständen möglich ist. Das vorgeschlagene Modell der Gesamtqualität eines Standortes, basierend auf verfügbaren Geodaten, wurde in verschiedenen Modellschritten implementiert, in denen jeweils unterschiedliche methodische Ansätze kombiniert werden. Das Modell führt pro Rasterzelle verschiedenste Geodaten zu einem dimensionslosen Zahlenwert als Summe der Lage-Indikatoren zusammen. Diese Zahlenwerte geben relativ zueinander eine Einstufung der „Standortqualität“ im Sinne der immobilienwirtschaftlichen „Lage“" an (Abb. 1).

Das Modell kann die Beurteilung der immobilienwirtschaftlichen „Lage“ unterstützen, aber nicht ersetzen. Eine Validierung und Optimierung des Modells anhand von Marktergebnissen ist unverzichtbar. Erste Untersuchungen konnten - durch das Überführen von Bodenrichtwerten und Angebotsdaten auf die Vektorgitterstruktur und den damit ermöglichten mathematischen Abgleich - die Ergebnisse für den Teilmarkt der Wohnimmobilien bereits plausibilisieren. 


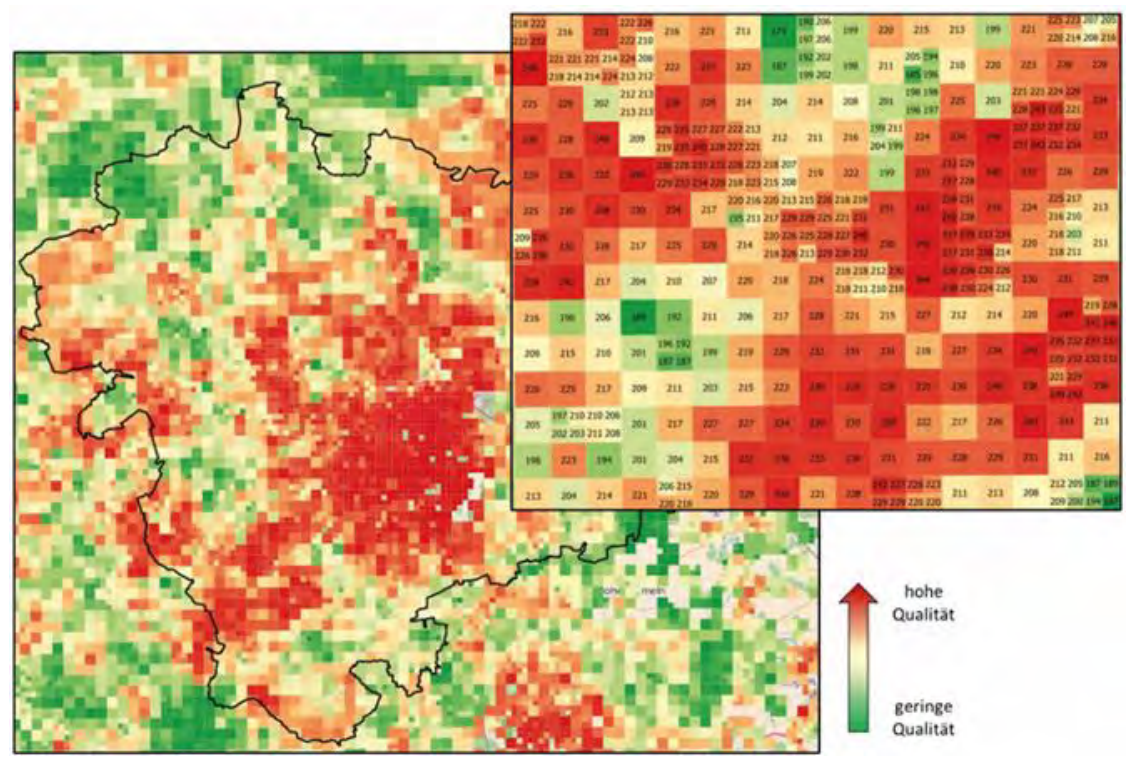

Abbildung 1: Auf Basis eines Vektorgitters dargestellte und berechnete Standortqualität für Wohnimmobilien (Region Hannover) (eigene Darstellung)

\section{Literatur}

Bakker, K., Voß, W. (2016): Modell zur Bestimmung der Standortqualität für Immobilien aus Geobasis- und Geofachdaten (Makroebene). Nachrichten der Nds. Vermessungs- und Katasterverwaltung, Heft 2/2016, S. 22-28.

Bill, R. (2016): Grundlagen der Geo-Informationssysteme. 6. Auflage Berlin/Offenbach: Wichmann Verlag.

EU-Richtlinie (2007): Richtlinie 2007/2/EG der Europäischen Parlaments und des Rates vom 14. März 2007 zur Schaffung einer Geodateninfrastruktur in der Europäischen Gemeinschaft (INSPIRE). 14.03.2007.

Fürst, D., Scholles, F. (2008): Handbuch Theorien und Methoden der Raum- und Umweltplanung. 3. Aufl., Dortmund: Verlag Dorothea Rohn.

Gudat, R., Voß, W. (2012): Endbericht zum Forschungsprojekt Weiterentwicklung der Markttransparenz am Grundstücks- und Immobilienmarkt. URL: https://www.gih.uni-hannover.de/uploads/tx_tkpublikationen/ endbericht markttransparenz 2012.pdf (last accessed 15.02.2017).

Ottmann, M., Lifka, S. (2010): Methoden der Standortanalyse, 1. Auflage. Schriftenreihe Geowissen kompakt, Wissenschaftliche Buchgesellschaft (WBG), Darmstadt. 
Voß, W., Bakker, K. (2017): Einsatz von Geodaten für die Immobilienwertermittlung. In: Meinel/ Schumacher/ Schwarz/ Richter (Hrsg.): Flächennutzungsmonitoring IX - Nachhaltigkeit der Siedlungs- und Verkehrsentwicklung? IÖR Schriften Band 73, Rhombus-Verlag Berlin, S. 113-122.

Zangenmeister, C. (1971): Nutzwertanalyse in der Systemtechnik: Eine Methodik zur multidimensionalen Bewertung und Auswahl von Projektalternativen, 2. Aufl., München: Wittemann Verlag. 



\title{
Daseinsvorsorgeatlas Niedersachsen - Stand und Ausblick eines Werkzeugs zur Steuerung und Planung von Daseinsvorsorgeeinrichtungen
}

\author{
Manfred Klaus, Stephan Mäs, Alexandra Weitkamp, Lars Bernard \\ TU Dresden, Professur für Landmanagement und Professur für Geoinformatik \\ Helmholtzstraße 10, 01069 Dresden \\ landmanagement@tu-dresden.de
}

\begin{abstract}
Demographischer Wandel, ökonomische Globalisierung und zum Teil desolate kommunale Finanzlagen stellen die Raumordnung vor große Herausforderungen. Gleichwertige Lebensverhältnisse können zukünftig nur durch die Anpassung der vorhandenen Infrastrukturen gewährleistet werden. Hierzu bedarf es eines Instrumentes zur Steuerung und Planung der Daseinsvorsorgeeinrichtungen. Der Daseinsvorsorgeatlas Niedersachsen bietet dafür Visualisierungs- und Analysemöglichkeiten hinsichtlich Bevölkerungszusammensetzung sowie zur Erreichbarkeit verschiedener Daseinsvorsorgeeinrichtungen wie Schulen, Kitas oder Arztpraxen. Aktuelle Entwicklungen sollen verschiedene Anwendungsgruppen aus Politik und Verwaltung durch die zusätzliche Integration einer multikriteriellen Analysemöglichkeit in die Lage versetzen, Monitoring und Standortplanungen für Daseinsvorsorgeeinrichtungen zielgerichteter durchzuführen.
\end{abstract}

\section{Daseinsvorsorge und gleichwertige Lebensverhältnisse}

Gleichwertige Lebensverhältnisse in allen Teilräumen des Bundesgebietes sind seit vielen Jahrzehnten Ziel der Raumordnung und Landesplanung. Das Ziel leitet sich aus dem Sozialstaatsprinzip nach Artikel 20 Grundgesetz (GG) ab und ist ebenso im Artikel 72 GG sowie im Raumordnungsgesetz (ROG) verankert (Kötter, 2016). Präzisierend fordert das ROG, dass in allen Regionen Deutschlands ausgeglichene soziale, infrastrukturelle, wirtschaftliche, ökologische und kulturelle Verhältnisse anzustreben sind ( $\$ 2$ ROG). Bund und Länder haben demnach mittels ordnungs- und entwicklungspolitischer Instrumente und Maßnahmen dafür zu sorgen, dass regionale Disparitäten abgebaut werden bzw. sich 
zumindest nicht verstärken (Kötter, 2016). Eine Teilaufgabe ist hierbei die Sicherung der Daseinsvorsorge.

Der grundlegende Gedanke der Daseinsvorsorge ist die Befriedigung der Grundbedürfnisse (Essen, Trinken, Schlafen, Wohnen, Sicherheit, Gesundheit usw.) und die Schaffung von Möglichkeiten für eine selbstbestimmte Lebensführung. In Verbindung mit der Vorstellung gleichwertiger Lebensbedingungen ist Daseinsvorsorge damit als öffentlich flächendeckende „Gewährleistung eines Angebotes ausgewählter, als lebensnotwendig eingestufter Güter und Dienstleistungen“ zu verstehen. Dazu zählen der Brand- und Katastrophenschutz, die Gesundheitsversorgung, Bildung, Post und Telekommunikation sowie die Nahversorgung, die Mobilität und der Rettungsdienst (ARL, 2016). Die Qualität dieser Leistungen richtet sich an anerkannte gesellschaftliche Standards, welche oft einen „Kompromiss zwischen den Bedürfnissen der Menschen und den finanziellen Möglichkeiten des Staates“ darstellen. Politik, Landes- und Regionalplanung legen Standard- und Mindestwerte fest und definieren damit Grundversorgung im Sinne einer akzeptablen Mindestqualität. Sollten die Mindestwerte in mehreren Bereichen unterschritten werden, sind gleichwertige Lebensverhältnisse zu bezweifeln (Kötter, 2016).

\section{Aktuelle Herausforderungen der Raumordnung}

Der demographische und soziale Wandel sowie die ökonomische Globalisierung und Flexibilisierung führen $\mathrm{zu}$ demographisch und ökonomisch wachsenden, schrumpfenden und stagnierenden Räumen (Abbildung 1). Um zukünftig die Daseinsvorsorge sicherzustellen, ist eine Anpassung der vorhandenen Infrastrukturen notwendig, was durch die vielerorts desolate kommunale Finanzlage erschwert wird.

Mindeststandards sind nur ein Baustein, um den Herausforderungen $\mathrm{zu}$ begegnen. Insbesondere die peripher gelegenen, dünn besiedelten Räume wie das südliche Niedersachsen stehen vor der Problematik der wirtschaftlichen Tragfähigkeit für die Basisinfrastruktur und der sich verschlechternden Erreichbarkeit von Daseinsvorsorgeeinrichtungen (Kötter, 2016).

Eine Stabilisierung bzw. Zuwanderung in den peripheren ländlichen Regionen wird ausbleiben. Ebenso ist eine Änderung des demographischen Wandels hin zu einer höheren Fertilitätsrate von mindestens 2,1 nicht wahrscheinlich (Petrenz und Weitkamp, 2018). 


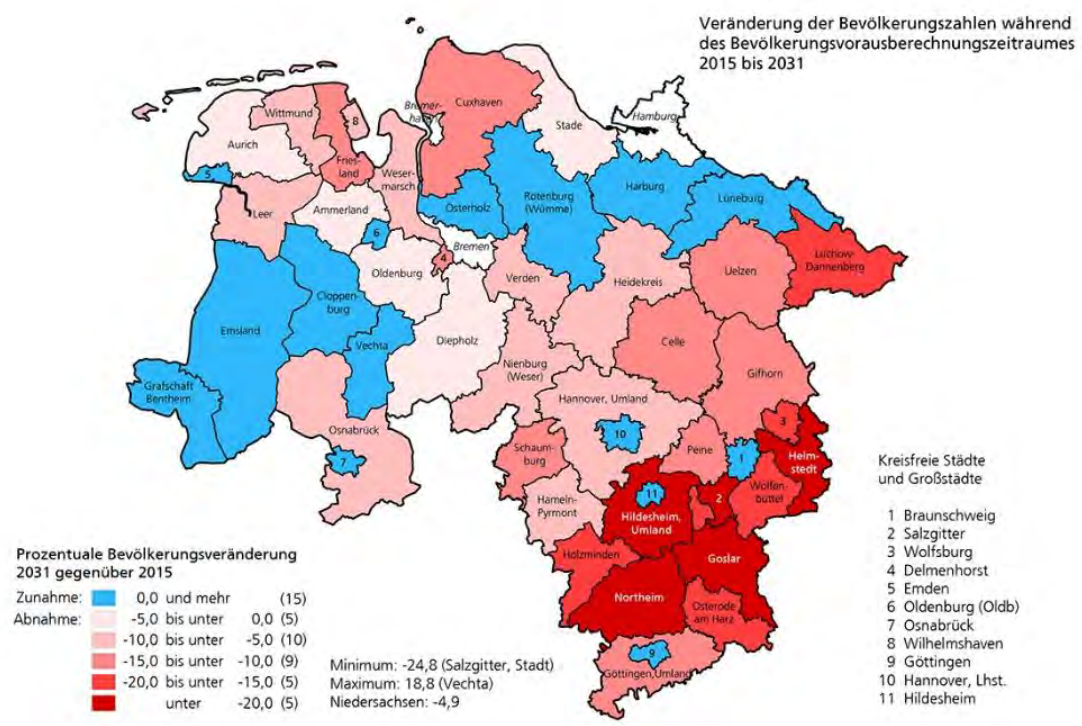

Abbildung 1: Veränderung der Bevölkerungszahlen in Niedersachsen während des Bevölkerungsvorausberechnungszeitraumes 2015 bis 2031 (Land Niedersachsen, o. J.)

Wie kann die Daseinsvorsorge angepasst und reorganisiert werden? Einen wichtigen Beitrag können neue digitale Möglichkeiten wie die Telemedizin, der lokale Online-Handel oder das E-Learning leisten (Hercksen, 2018). Für die Planungsebenen bedarf es allerdings eines Instrumentes, das die Versorgung der Regionen mit Daseinsvorsorgeeinrichtungen transparent darlegt, Defizite aufzeigt und Prognosen für etwaige Erweiterung und Veränderungen ermöglicht. Voraussetzungen dafür sind Informationen zu Daseinsvorsorgeeinrichtungen, deren Erreichbarkeit mit verschiedenen Verkehrsmitteln sowie Bevölkerungsdaten. Ohne einheitliche, vollständige und aktuelle Geodaten sind Planungen im Bereich der Daseinsvorsorge jedoch nicht möglich.

\section{Daseinsvorsorgeatlas Niedersachsen}

Ein Prototyp zur Abbildung und Steuerung der Daseinsvorsorgesituation ist der Daseinsvorsorgeatlas Niedersachsen (DVAN). Dieser wurde im BMBF-Projekt UrbanRural SOLUTIONS unter Leitung der TU Hamburg-Harburg in Koopera- 
tion mit dem Land Niedersachsen im Zeitraum 2017 bis 2019 entwickelt. Als Webapplikation können verschiedene Kartenlayer zu Themen wie Einzelhandel, Arztpraxen oder Schulen visualisiert, thematische Informationen zu einzelnen Objekten abgefragt oder mit den Bevölkerungsdaten, basierend auf den Zensus 2011, überlagert werden. Der Kern des Prototyps ist eine Erreichbarkeitsanalyse. Mit dieser können die zeitliche Erreichbarkeit oder der Bevölkerungsanteil, der innerhalb einer bestimmten Zeit eine Daseinsvorsorgeeinrichtung erreichen könnte, für den Modal Split berechnet und dargestellt werden. So kann beispielsweise die Pkw-Fahrzeit zu Hausärzten ermittelt werden (Abbildung 2) oder die Schüleranzahl einer Grundschule, die maximal ein Kilometer zu Fuß zurücklegen muss (Abbildung 3).

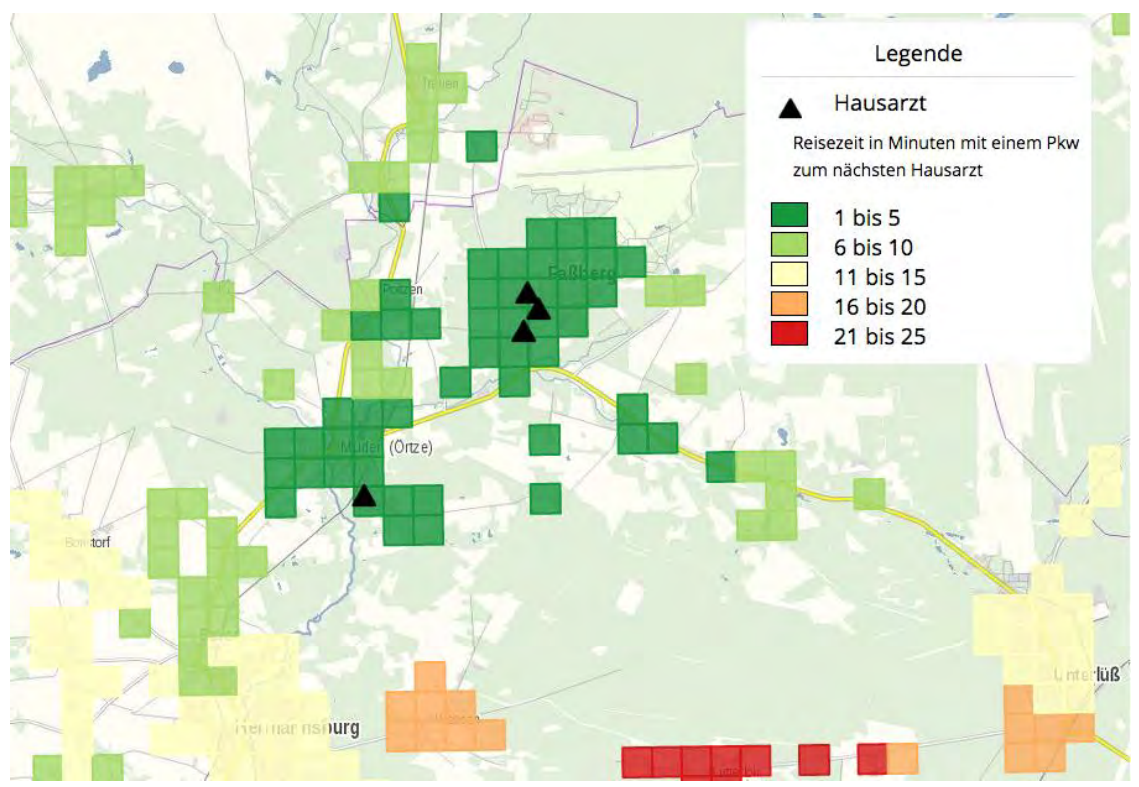

Abbildung 2: Erreichbarkeitsanalyse des DVAN - Reisezeit in Minuten mit dem Pkw zum nächstgelegenen Hausarzt (Klaus u. a., 2019) 


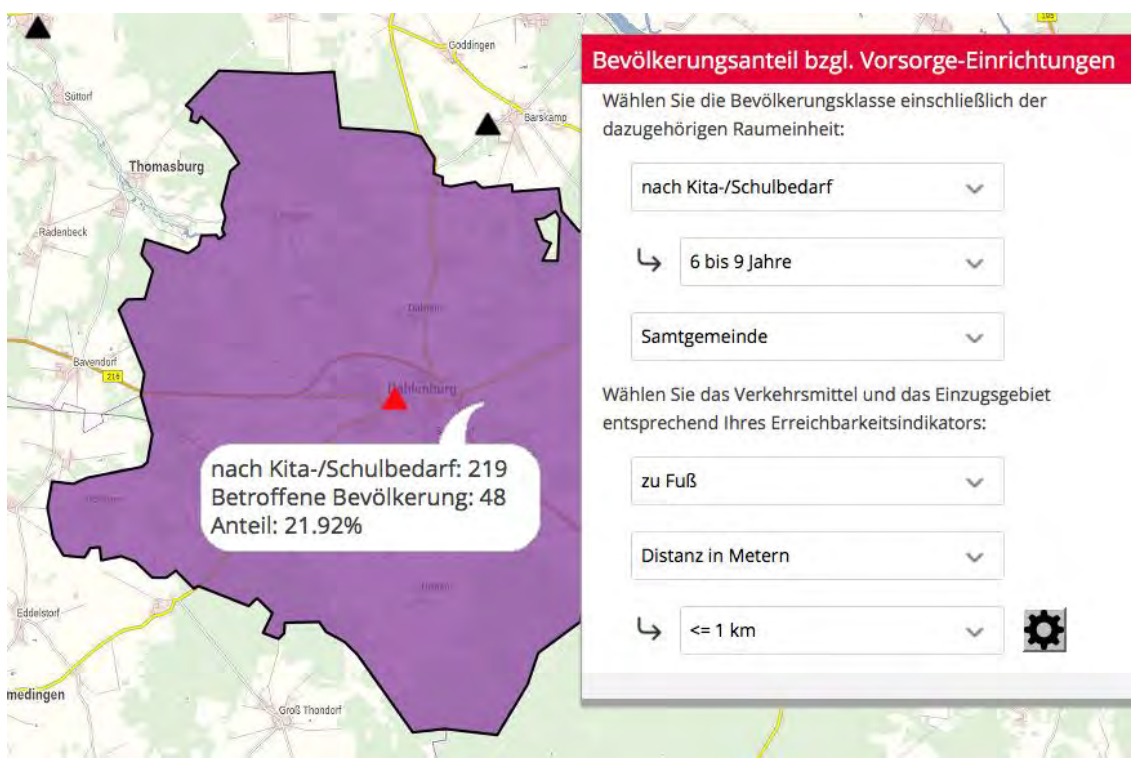

Abbildung 3: Beispiel Bevölkerungsanalyse in Abhängigkeit der Erreichbarkeit Anteil der Bevölkerung im Grundschulalter, die einen Fußweg zur zugehörigen Grundschule von maximal einem Kilometer haben

\section{Weiterentwicklungen des DVAN}

Die Freischaltung des aktuellen DVAN für die kommunalen Verwaltungsbehörden, der niedersächsischen Schulbehörde und ggf. der kassenärztlichen Vereinigung Niedersachsen ist für Mitte 2020 avisiert. Bereits seit September 2019 besteht eine neue Forschungskooperation zwischen der TU Dresden (Professur für Landmanagement, Professur für Geoinformatik) mit dem Land Niedersachsen. Diese hat zum Ziel, den jetzigen Prototyp des DVAN bei der Einführung zu begleiten und anschließend zu einem umfassenden Planungsunterstützungstool auf Basis des Masterportal Hamburgs weiterzuentwickeln. Hierbei soll auch der gegenwärtige Anwenderkreis um weitere Anwendungsgruppen erweitert werden.

Zukünftig soll der DVAN eine multikriterielle Entscheidungsunterstützung bieten. Die Nutzer sollen in die Lage versetzt werden, Standortvergleiche anhand von selbstgewählten Kriterien unterschiedlicher Gewichtung zu tätigen. Fachplaner werden dabei unterstützt, je nach Vorgaben optimale Lagen zu be- 
stimmen. So könnte beispielsweise eine Gemeinde neue Standorte des Gerätehauses der Freiwilligen Feuerwehr in Abhängigkeit von der festgelegten Hilfsfrist (beinhaltet u. a. die Ausrückzeit zum Gerätehaus als auch Anfahrtszeit zur Einsatzstelle) sowie der Siedlungsstruktur bestimmen: Innerhalb einer definierten Zeit sollen $100 \%$ der Bevölkerung der Gemeinde einschließlich der Grundschule auf dem zweiten Rettungsweg erreicht werden. Ein weiteres Planungsszenario wäre die interaktive Ermittlung der Schulstandorte anhand der Kriterien Fahrzeit mit dem ÖPNV, aktueller und zukünftiger schulpflichtiger Bevölkerungszusammensetzung sowie der freien Kapazitäten der umliegenden Schulen.

Die Ebene der Regionalplanung profitiert beim Ausbau des DVAN zu einem multikriteriellen Entscheidungsunterstützungstool, u. a. durch die Möglichkeit der Identifizierung von Räumen mit Handlungsbedarf.

Ein weiterer wesentlicher Entwicklungsschritt ist die Anpassung des DVAN an Nutzergruppen. Durch die Erweiterung des Anwenderkreises ist es aufgrund unterschiedlicher Expertise notwendig, eine Basisvariante und einen Expertenmodus zu entwickeln. Daraus ergeben sich unterschiedliche Anforderungen an den DVAN. Während im Expertenmodus erhöhte Anforderungen an Zuverlässigkeit und Funktionalität bestehen, ist in der Basisvariante eine möglichst intuitive Benutzbarkeit wichtig. Diese umfasst insbesondere die Ästhetik, die Fehlertoleranz sowie die Bedienbarkeit und Erlernbarkeit des Systems.

\section{$5 \quad$ Zusammenfassung}

Daseinsvorsorge und gleichwertige Lebensverhältnisse sind relevante gesellschaftliche Themen. Die demographische Entwicklung, die ökonomische Globalisierung und Flexibilisierung, die Digitalisierung und die vielerorts desolate kommunale Finanzlage stellen Politik und Verwaltung vor große Herausforderungen. Nur durch Anpassung der vorhandenen Infrastrukturen an neue Gegebenheiten kann die Versorgung der Bevölkerung sichergestellt werden. Hierzu bedarf es Instrumenten zur Steuerung und Planung von Daseinsvorsorgeeinrichtungen. Diese Aufgabe soll zukünftig der Daseinsvorsorgeatlas Niedersachsen (DVAN) einnehmen, der derzeit als Webapplikation zur Visualisierung von Kartenlayern zu verschiedenen Themen wie Einzelhandel und Ärzte sowie zur Berechnung von Erreichbarkeiten für verschiedene Bevölkerungsgruppen dient. In einer Forschungskooperation zwischen der TU Dresden und dem Land Niedersachsen wird der DVAN zu einem multikriteriellen Entscheidungsunterstützungstool weiterentwickelt. Durch den Ausbau der Funktionalität steht zu- 
künftig eine umfassende und zielführende Steuerungsmöglichkeit der Daseinsvorsorge zur Verfügung.

\section{Literatur}

Akademie für Raumforschung und Landesplanung (ARL) (2016): Daseinsvorsorge und gleichwertige Lebensverhältnisse neu denken - Perspektiven und Handlungsfelder. Positionspapier aus der ARL 108

Hercksen, H. (2018): Digitalisierung als Chance für die Daseinsvorsorge in ländlichen Räumen. Flächenmanagement und Bodenordnung (fub), Ausgabe 2/2018

Klaus, M., Mäs, S., Bernard, L., Weitkamp, A. (2019): Weiterentwicklung des Daseinsvorsorgeatlas Niedersachsen. Nachrichten der Niedersächsischen Vermessungs- und Katasterverwaltung (NaVKV), Ausgabe 1+2/2019

Kötter, T. (2016): Gleichwertigkeit der Lebensbedingungen - Herausforderungen und Ansätze für eine wichtige Aufgabe der Raumordnung. Flächenmanagement und Bodenordnung (fub), Ausgabe 4/2016

Land Niedersachsen: Der demografische Wandel in Niedersachsen. https://www.niedersachsen.de/startseite/themen/demografie_und_regionalentwi cklung/dynamisches_niedersachsen/demografie_niedersachsen/demografischer -wandel-in-niedersachsen-146677.html, aufgerufen am 18.02.2020

Petrenz, J., Weitkamp, A. (2018): Wüstungen als mögliche Folge von Schrumpfungsprozessen sowie offene Fragen zum Umgang mit ihnen. Flächenmanagement und Bodenordnung (fub), Ausgabe 2/2018 

Sicherheit 



\title{
Vernetzung von mobil und stationär gewonnenen Schadstoffwerten bei großflächigen ABC-Gefahrstofffreisetzungen
}

\author{
Christoph Averdung \\ CPA ReDev GmbH \\ Auf dem Seidenberg 3a, D-53721 Siegburg \\ ca@supportgis.de
}

\begin{abstract}
Der Beitrag beschreibt die integrative Vernetzung von mobil gewonnenen Schadstoffwerten mit den stationär erfassten Planungs- und Lageinformationen bei ABC-Gefahrstofffreisetzungen. Ein besonderer Fokus liegt dabei sowohl auf dem rein digitalen Informationsfluss zwischen Einsatzleitung, Messleitung und mobilen Schadstoffmesstrupps wie auch der zeitbezogenen Verwaltung aller bei einem großflächigen Schadensereignis gemessenen Messwerte und den aus diesen Werten abgeleiteten Dokumenten.
\end{abstract}

\section{$1 \quad$ Einleitung}

Bei einem Austritt chemischer, biologischer und atomarer Stoffe stehen die rasche Aufklärung des räumlichen Schadensbereichs wie auch die qualifizierte Information betroffener Bevölkerungsteile im Mittelpunkt des Handelns. Dies geschieht mit dem Ziel, eine Gefährdung der Bevölkerung, der Umwelt und von Sachwerten so gering wie möglich zu halten. Der Faktor „Zeit“ ist dabei eine wesentliche Einflussgröße; sowohl bei der Ermittlung von Schadstoffwerten vor Ort wie auch bei der nachfolgenden Ableitung von Sachinformationen, Kartendarstellungen und Reports für die Dokumentation des Ereignisses.

Die Leistungsanforderungen an die Gefahrenabwehr bei einer großflächigen ABC-Gefahrstofffreisetzung sind für die Kreise und kreisfreien Städte im Bundesland Nordrhein-Westfalen im „ABC-Schutz-Konzept NRW“ [ABC-1] dokumentiert. Diese Spezifikationen bilden die Grundlage für einen digitalen Informationsfluss, wie er idealerweise bei einem Großschadensereignis umgesetzt wird. Im Mittelpunkt der folgenden Betrachtungen stehen diejenigen Prozess- 
schritte, die eine Einsatzleitung mit belastbaren Informationen zum Gefahrenbereich und zum Ausbreitungsverhalten der Gefahrstoffe versorgt. Dies sind insbesondere

- die räumliche Lokalisation und sachbezogene Dokumentation der Schadensmeldung,

- die Definition des Gefahrenbereichs, das Bewerten des Ausbreitungsverhaltens und das Erstellen einer Ausbreitungsprognose,

- das sich an der Ausbreitungsprognose orientierende Festlegen von vor Ort identifizierbaren Messpunkten und deren Übertragung an die mobilen Messtrupps,

- die Entgegennahme der Messdaten, deren Auswertung in Richtung einer Schadstoffwolke,

- $\quad$ die Übertragung weiterer Messaufträge an die Messtrupps (aufgrund der aus den Messdaten aktuell abgeleiteten Schadstoffwolke),

- die kontinuierliche Produktion von Karten und Reports für die Einsatzleitung und

- $\quad$ die Archivierung aller gewonnenen Daten und Produkte (Messort und Messdaten, Ausbreitungsprognose, Schadstoffwolke(n), Reports).

Sowohl die Messdaten wie auch die daraus abgeleiteten Produkte werden mit einem individuellen Zeitbezug versehen. Damit steht auch im Nachhinein eine lückenlose Historie des Ausbreitungsverhaltens der Gefahrstoffe zur Verfügung. Sie kann z. B. einer fehlerhaften Berichterstattung entgegenwirken oder als fundierte Ausbildungsgrundlage für eine zukünftige Gefahrenabwehr dienen.

Die folgenden Ausführungen stellen die Prozesskette zur mobilen Messung von Gefahrstoffen und deren Auswertung vor, wie sie CPA zusammen mit der Kreisfeuerwehr Recklinghausen und den Feuerwehren der kreisangehörigen Gemeinden entwickelt.

\section{Informations- und Entscheidungsplattform}

Als Plattform für die Datengewinnung und Auswertung der Gefahrstoffmessungen werden SGJ-GeoHornet als webbasiertes GIS und SGJ-MobileGIS als mobile Android-App eingesetzt. Beide Softwareprodukte verfügen über die erforderlichen Voraussetzungen für die Umsetzung der beschriebenen Teilaspekte einer Prozesskette, die von der Digitalisierung der Messwerte und der Ausbreitungsprognose über die eigentliche Datengewinnung mit einer bidirektionalen 
Kommunikation zwischen Messleitung und Messtrupp und der Ableitung der tatsächlichen Schadstoffwolken reicht.

Da wesentliche Teile der Aufgabenstellung durch die Konfiguration der beiden Softwareprodukte gelöst werden konnten, entstanden bereits zu einem frühen Zeitpunkt in dem Projekt einzelne Prototypen, die nahezu der gewünschten Lösung entsprachen und zudem das Fehlen einzelner Aspekte aufzeigen konnten. So wurden zunächst als Grundlage für die Abbildung der Einzelfunktionen die folgenden Eigenschaften beider Softwareprodukte herangezogen:

- ISO/OGC-konforme Modellierung der Datenbasis. Es wurde ein sich im Wesentlichen an den Dokumenten „ABC-Schutz-Konzept NRW“ [ABC-1] und „ABC-Messstrategie NRW“ [ABC-2] orientierendes Fachdatenschema erstellt, um darüber die SGJ-Database unter PostgreSQL (für SGJ-GeoHornet) und SQLite (für SGJ-MobileGIS) identisch aufzubauen.

Damit sind die Voraussetzungen für eine barrierefreie Datenübertragung zwischen dem stationären und mobilen GIS gegeben. Als Datenschnittstelle fungiert die Geographic Markup Language (GML); die Fortführungslogik zwischen den beiden Systemen erfolgt über GML-Fortführungsdatensätze analog zu einem Web Feature Service Transactional (WFS-T).

- Der Erfassungsdialog in SGJ-MobileGIS orientiert sich an dem ISOOGC-konformen Fachdatenschema der SGJ-Database. Per Konfiguration kann dieser Dialog gezielt auf den Anwendungsfall hin angepasst werden. So lassen sich z. B. Auswahllisten an Schadstoffen, Grenzwerte für die Datengewinnung oder einfache mit Ja oder Nein zu beantwortende Fragen definieren, um darüber den Erfassungsprozess deutlich zu vereinfachen. Auch SGJ-GeoHornet benutzt dieselbe Konfigurationstechnologie, sodass die Messdaten auf einfachste Art und Weise stationär nachdigitalisiert werden können, z. B. wenn der automatisierte Datenabgleich zwischen stationärem und mobilem System ausfällt und die Daten per (digitalem) Sprechfunk übermittelt werden müssen.

- Für den Datenaustausch zwischen dem stationärem SGJ-GeoHornet und den mobilen Systemen der Messtrupps (SGJ-MobileGIS) sorgt mit nextcloud eine cloudbasierte Lösung der GKD Recklinghausen. Diese Speicherlösung wird unter den Betriebssystemen MS Windows und Google Android für den gegenseitigen Austausch von GMLFortführungsdatensätzen eingesetzt. Über die gezielte Konfiguration 
von Datenaustauschverzeichnissen wird der Aufwand für den Datenbezug auf beiden Seiten der Systeme minimiert.

Die durch diese Eigenschaften erworbenen Vorteile waren im Hinblick auf eine einfache Benutzerführung weiter zu stärken, insbesondere vor dem Hintergrund, dass die Anwender nur über geringe bis keine GIS-Kenntnisse verfügen und unter Einsatzbedingungen komplex strukturierter Anwendungen keine Akzeptanz finden. Daher wurden einige zentrale Aspekte der Aufgabenstellung individuell als Plugin programmiert und entsprechend in der Laufzeitumgebung von SGJ-GeoHornet verfügbar gemacht. Dabei handelt es sich um

- die Konstruktion der Ausbreitungsprognose als Ausbreitungskeule,

- die Digitalisierung der Messpunkte einschließlich deren attributiver Vorbelegung als Arbeitsvorbereitung für die Messtrupps vor Ort,

- die Digitalisierung der Schadstoffwolke auf der Grundlage erhaltener Gefahrstoffmessungen und

- die dialoggestützte Kommunikation mit den Messtrupps über die nextcloud und ihrer WebDAV-Schnittstelle.

Die Funktionsweise der getroffenen Systemkonfiguration und -programmierung beschreibt das nächste Kapitel.

\section{Datenkommunikation}

Nach der Lokalisation des Schadensereignisses wird auf der Grundlage des austretenden Gefahrstoffes (Kontamination) und der lokal herrschenden Witterungsbedingungen eine Schadstoffwolke als Ausbreitungsprognose digitalisiert. Die Parameter dafür sind der Radius der allseitig vorzunehmenden Sperrung sowie der Radius der Beeinträchtigung in Windrichtung.

Nach dem Vorliegen der Ausbreitungsprognose erfolgt für die einzelnen Messtrupps die Digitalisierung der Messpunkte in Form von dialoggestützt bereitgestellten Erfassungstemplates. So werden an dieser Stelle bereits alle zur Orientierung dienenden Attributwerte erfasst, z. B. die Bezeichnung des die Messung ausführenden Messtrupps, der Ort der Messung, die Art des austretenden Gefahrguts oder das zum Einsatz kommende Messgerät. Im Anschluss daran wird die Übertragung der Ausbreitungsprognose wie auch der Messpunkttemplates in Richtung des mobil eingesetzten Tablets angestoßen. 


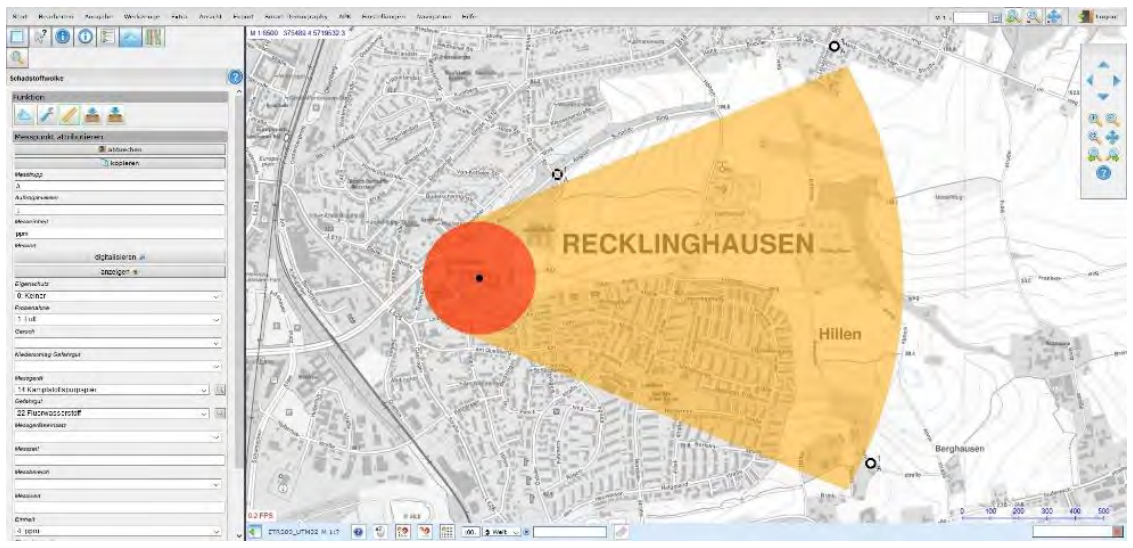

Abbildung 1: Stationäre Digitalisierung der Ausbreitungsprognose und der Messpunkte

Der für die Messung zuständige Messtrupp übernimmt per GML-Import die Ausbreitungsprognose und gezielt die für ihn bestimmten Messpunkte in die mobile SGJ-Database von SGJ-MobileGIS. Eine Styled Layer Description (SLD)-Konfiguration sorgt im Anschluss daran für die Visualisierung der erhaltenen GML-Daten. Auf dieser Arbeitsgrundlage basiert die nachfolgende Messung der Schadstoffwerte. Der Messtrupp geht oder fährt die Messpunkte ab und erfasst die fehlenden Attribute.

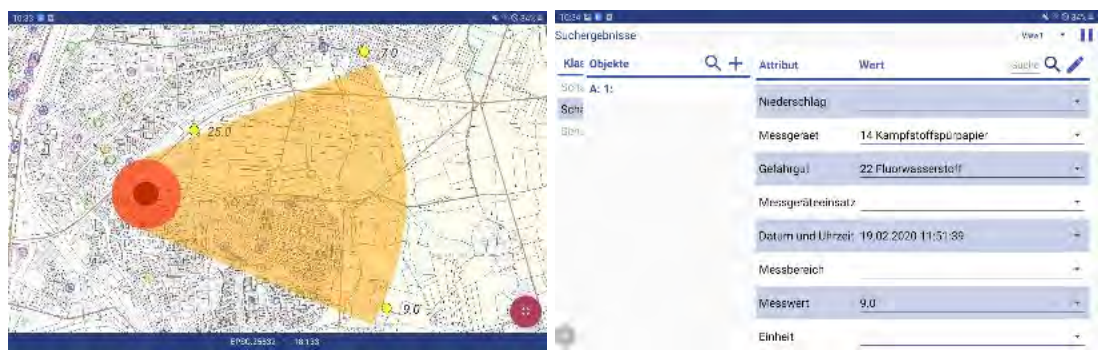

Abbildung 2: Mobile Kartendarstellung und Formular zur Messdatengewinnung

Theoretisch ist dabei der Messtrupp in der Lage, auch neue Messpunktorte und Messdaten konform zum Fachdatenschema des Einsatzlagezentrums zu digitalisieren. Nach dem Abschluss der Messtätigkeiten werden alle vor Ort gewonnenen Informationen als GML-Update-Datensatz über die nextcloud an die Messleitung gesandt und dort in die zentrale SGJ-Database hinein übernommen. 
Auf der Grundlage der Messdaten und ggf. der Daten eines im Kreisgebiet verorteten und in SGJ-GeoHornet eingebundenen LoRaWAN-Sensornetzwerks wird dann die eigentliche Schadstoffwolke in SGJ-GeoHornet manuell digitalisiert. Die aus den Messdaten abgeleiteten Einsatztoleranzwerke bzw. der ermittelte AEGL (Acute Exposure Guidline Level)-Schweregrad führen zu einer kartografischen Darstellung der Schadstoffwolke mit roten (schwerwiegend, irreversible Schäden an der Gesundheit) und gelben unterschwelligen Flächenanteilen.

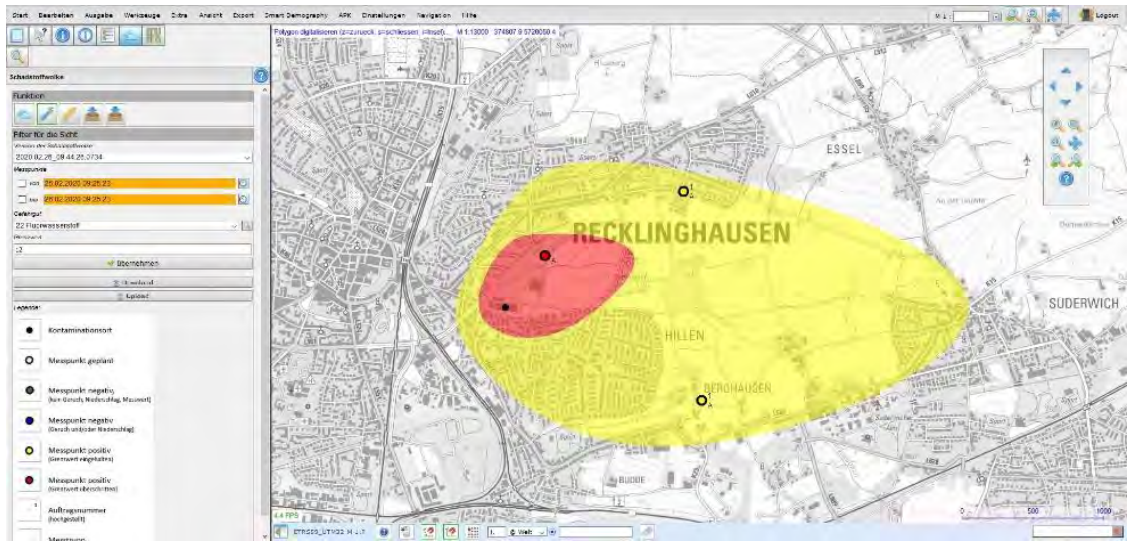

Abbildung 3: Digitalisierte Schadstoffwolke der Messleitung

Am Ende dieses Prozesses wird die aus den Messdaten abgeleitete Schadstoffwolke gegenüber der Einsatzleitung als digitale Karte veröffentlicht. Dies geschieht in Form eines OGC-konformen Web Map Service (WMS), der ohne weiteren Installationsaufwand über SGJ-GeoHornet bereitgestellt wird. Kommt die Einsatzleitung zu der Erkenntnis, die Lage über weitere Messungen aufklären zu müssen, werden weitere Messpunkte festgelegt und an die Messtrupps übertragen. Diese ergänzen ihren mobilen Datenbestand und senden nach den Messaktivitäten erneut einen GML-Update-Datensatz an die Messleitung zurück. Diese erstellt auf dieser neuen Beurteilungslage eine neue Schadstoffwolke. 


\section{Dokumentation, Datensicherung und Archivierung}

Die Messdaten und die dazu passenden Schadstoffwolken werden jeweils mit einem Zeitbezug gespeichert. Dasselbe gilt für die Ausbreitungsprognose. Damit können alle bei der Gefahrstoffmessung durchgeführten Aktivitäten lückenlos in der SGJ-Database dokumentiert und archiviert werden. Als zusätzliches Element einer Datensicherung lassen sich die Ausbreitungsprognose, die gewonnenen Messdaten und die aus ihnen gewonnenen Schadstoffwolken als GML-Datenbestand exportieren. Zusammen mit dem dazu passenden Fachdatenschema kann über viele Jahre hinweg ein automatisiert lesbarer Datensatz für die Rekonstruktion des Schadensereignisses hergestellt werden. Davon unabhängig werden aus den gewonnenen Daten digitale und analoge Kartenprodukte und Messprotokolle abgeleitet. Diese eignen sich sowohl für die Dokumentation wie auch zur Langzeitarchivierung.

\section{$5 \quad$ Zusammenfassung}

Die Veröffentlichung stellt ein hochgradig vernetztes Geoinformationssystem für das Informationsmanagement von vernetzt gewonnenen Messdaten bei einer z. B. großflächigen ABC-Gefahrstofffreisetzung vor. Die durch Messtrupps über mobile GIS-Technologie digitalisierten und per Daten-Cloud übertragenen Schadstoffwerte werden zusammen mit den hochfrequent bereitstehenden Messdaten flächenhafter Sensornetzwerke in Echtzeit über ein webbasiertes GIS registriert, teil- und vollautomatisch in digitale Produkte wie OGC-konforme Kartendienste überführt und anschließend zum Zwecke der vollständigen Dokumentation des Schadensereignisses archiviert.

\section{Literatur}

[ABC-1] ABC-Schutz-Konzept NRW - Teil $5 »$ Messzug NRW«, Ministerium für Inneres und Kommunales des Landes Nordrhein-Westfalen, Dezember 2011

[ABC-2] ABC-Messstrategie, Institut der Feuerwehr Nordrhein-Westfalen, Lernunterlage B3-021, März 2017 



\title{
Brandschutzbedarfsplanung in Mecklenburg- Vorpommern mithilfe amtlicher Geodaten
}

\author{
Jennifer Wurlich, Marco L. Zehner \\ DVZ Datenverarbeitungszentrum Mecklenburg-Vorpommern GmbH \\ j.wurlich |m.zehner@dvz-mv.de
}

\begin{abstract}
Für viele verwaltungstechnische Fragestellungen werden vorhandene Geobasis- und Geofachdaten von jeweils anderen Verwaltungen benötigt. Hierfür stehen die Daten und Lösungen im Rahmen der GDIMV bereit. Dieser Beitrag beschäftigt sich mit den Grundsätzen, wie Geoinformationen automatisiert aus amtlichen elektronischen Quellen zusammengetragen und den Anwendern für Auswertungen bereitgestellt werden können. Konkret wird dies am Praxisbeispiel Brandschutzbedarfsplanung umgesetzt, wofür regelmäßig zahlreiche und verschiedenartige Kennzahlen und Informationen für die Risikobewertung und die Planung zusammengestellt werden müssen.
\end{abstract}

\section{$1 \quad$ Praxisbeispiel Brandschutzbedarfsplanung}

Das Feuerwehrwesen ist in der Bundesrepublik Deutschland landesrechtlich geregelt. Der Aufgabenbereich geht in der Regel über die Brandbekämpfung auf sonstige Unglücks- und Notfälle hinaus. In Mecklenburg-Vorpommern werden der Brandschutz und die Hilfeleistung durch das Gesetz über den Brandschutz und die Technischen Hilfeleistungen durch die Feuerwehren für MecklenburgVorpommern geregelt. Nach der Verwaltungsvorschrift für Brandschutzbedarfspläne (VV Meckl.-Vorp. Gl. Nr. 2131 - 9 vom 12. Oktober 2017 - II 450) sollen die Gemeinden nach allgemeingültigen Regeln und unter Beachtung der Besonderheiten des Gemeindegebietes die Ausstattung und die Leistungsfähigkeit ihrer Feuerwehr festlegen und die danach erforderlichen Maßnahmen veranlassen. Die erforderlichen Angaben sind regelmäßig bei Änderungen und maximal alle 5 Jahre fortzuschreiben.

Neben einer Erhebung und Beschreibung für eine Gefährdungsabschätzung und Gefahrenabwehrplanung ist die eigentliche Risikoabschätzung und zugehörige 
Planung Bestandteil der Brandschutzbedarfsplanung. Für die Beschreibung des Gefährdungspotenzials sind gemäß der Anlage 1.2 der VV zahlreiche Angaben zu erheben. Hierzu gehören Gemeindestruktur, Flächennutzung, Verkehrsinfrastruktur, Bebauung, bauliche Objekte, Industrie und Gewerbe und Versorgungseinrichtungen. Diese Daten liegen in den Geodatenbeständen der Landes- und Kommunalverwaltungen vor. Für den nachhaltigen Einsatz ist das Ziel, alle relevanten Daten jeweils automatisiert aus den originären Quellen abzurufen und eine Planungsvorlage zu generieren.

\section{Anforderungen und Ziele}

Die Anwendung für die Brandschutzbedarfsplanung ist ein Beitrag zur Umsetzung der Ziele der Nationalen Geoinformationsstrategie (NGIS) und wird im Rahmen des Umsetzungsprojekts für Mecklenburg-Vorpommern durchgeführt. Aus diesem Anlass bestehen nicht nur funktionale, sondern auch konzeptionelle Anforderungen.

\subsection{Anforderungen Ziele NGIS}

NGIS hat drei Grundsätze: die Grundversorgung zu sichern, die Mehrfachnutzung zu erleichtern und Innovationen zu fördern. Dies soll die Lösung exemplarisch darstellen und somit ist aufzuzeigen, welche Vorteile im Sinne der Ziele von NGIS erzielt werden können, welche Herausforderungen und Lösungen es gibt und wie die Vorgehensweise ebenfalls von weiteren Verwaltungsfachverfahren genutzt werden kann. Erste konkrete Ziele für die Verwaltungen wurden wie folgt definiert:

- Daten werden nicht neu und redundant erhoben.

- Die datenhaltenden Stellen werden durch automatisierten Abruf entlastet.

- $\quad$ Für die anfragenden Verwaltungen entfällt eine Wartezeit.

- Die Nutzung des zentralen Werkzeuges für die Datenerhebung stellt eine Arbeitserleichterung dar, da die Identifizierung einmalig durch den Verfahrensinhaber und die Zusammenstellung der Daten durch das Werkzeug übernommen wird.

- Die zentrale Bereitstellung der Geoinformationen aus definierten Quellen sichert die Qualität der Daten.

- Betrachtungen im Rahmen der Brandschutzbedarfsplanung sind untereinander vergleichbar. 


\subsection{Anforderungen Brandschutzbedarfsplanung}

Die Anforderungen zur Brandschutzbedarfsplanung ergeben sich aus den technischen Zielen zur Digitalisierung sowie aus der Fachlichkeit. Bei den ITVerfahren gilt das Gebot der Nach- bzw. Mitnutzung von bestehenden Lösungen sowie der Aufbau von sicheren und einfach zu bedienenden Umgebungen.

Als Ergebnis der ersten Umsetzungsphase soll die öffentliche Stelle ein vordefiniertes Dokument mit allen verfügbaren Informationen bereitgestellt bekommen, welches für die weitere Risikoanalyse und Planung direkt genutzt werden kann. Zur Grundanforderung gehört der Datenabruf aus den definierten Quellen. Diese Quellen müssen technisch erreichbar und auswertbar sein, um nur die relevanten Informationen zu erhalten.

Eine wichtige Funktion ist hier die räumliche Verschneidung und Aggregation. Zum Beispiel ist für einen Ortsteil die Information wichtig, wie viele Gebäude höher $12 \mathrm{~m}$ vorhanden sind, um die notwendige Rettungstechnik einzuplanen. Eine weitere Auswertung in der Planung sind Kartendarstellungen. Für einzelne Bereiche werden Kartenausschnitte benötigt. Neben dem Kartenausschnitt für Hintergrunddaten sind die Fachobjekte auf der Karte darzustellen. Für weitere Datenabfragen und Analysen (z. B. Fahrzeitanalyse) sind gegebenenfalls speziell definierte Schnittstellen erforderlich und entsprechend zu integrieren.

\section{Umsetzung der Komponenten}

Für die Erstellung der Vorlage sind verschiedene Schritte notwendig, nach der die Anwendung umgesetzt wurde:

- $\quad$ Authentifizierung/Autorisierung des Nutzers, da nicht alle Daten frei zur Verfügung stehen.

- $\quad$ Auswahl der Gemeinde/Verwaltungseinheit, für die die Vorlage erstellt werden soll.

- $\quad$ Abruf der definierten Daten und Geodaten, Analyse und Zusammenstellung der Ergebnisse.

- $\quad$ Erstellung der Vorlage und Befüllung der gemeindebezogenen und ermittelten Daten.

- Bereitstellung der Vorlage für den Nutzer zum Download. 


\subsection{Realisierung im Rahmen GDI-MV}

Die Lösung wird vollständig in den zentralen Infrastrukturknoten der GDI-MV integriert, um vorhandene Komponenten mit zu nutzen und um direkt auf vorliegende Daten zugreifen zu können. Im Einzelnen sind dies:

- $\quad$ Technische Infrastruktur, wie Datenbank, Server und Loadbalancer.

- $\quad$ Softwarebibliotheken, mit GIS-Funktionen zum Abruf und zur Analyse.

- Lokale Geodatenbestände im Rahmen des Infrastrukturknotens (Geodatenbank, Rasterkarten)

- $\quad$ GeoPortal.MV Nutzer- und Rechteverwaltung

- Geodatenviewer zur Auswahl der Gemeinde.

- Downloadbereich für registrierte Nutzer.

Die zentrale Nutzer- und Rechteverwaltung im GeoPortal.MV ermöglicht es fachverfahrensübergreifend, den Zugriff auf Anwendungen und Datenbestände zu steuern. So wirken jeweils die Freigaben der Anwendungs- und Dateneigentümer auch bei der Brandschutzbedarfsplanung. Der Downloadbereich für registrierte Nutzer wird benötigt, weil die Datenzusammenstellung und Analyse längere Zeit benötigt und der ausschließliche Zugriff des Nutzers garantiert werden soll.

\subsection{Modul Brandschutzbedarfsplanung}

Die Grundfunktionen der Lösung wurden als eigenständiges Modul umgesetzt. Aus Sicht des Nutzers und der Rahmenanwendung ist es ein abgeschlossener Prozess. Das Modul kann damit in beliebige Umgebungen eingebettet werden. Eingangsparameter für den Prozess sind nur der eindeutige Schlüssel der Verwaltungseinheit und ggf. Hinweise zum Datenzugriff, sofern gesonderte Rechte vorliegen. Aktuell wird das Modul im Geodatenviewer GAIA-MV GDI des Geo-Portal.MV gestartet. Nach Anmeldung und Auswahl einer Gemeinde erfolgt der Start der Aufbereitung.

Im Modul werden als Erstes die konfigurierten Daten abgerufen und ausgewertet. Hierbei werden technologisch verschiedene Datenquellen angesprochen und gleich zur Analyse genutzt:

- $\quad$ Direktzugriff Geodatenbank (PostgreSQL/PostGIS)

- $\quad$ Abruf externer Web Feature Server (WFS)

- $\quad$ Hochaufgelöste Web Map Server (WMS) zur Kartenerzeugung in Druckqualität 


\section{- Web Prozess Service (Routenermittlung)}

Die Nutzer werden per E-Mail darüber informiert, dass die Erstellung einer Vorlage zum Brandschutzbedarfsplan fertiggestellt wurde. Die E-Mail enthält einen Link, über welchen das Dokument heruntergeladen werden kann.

\section{Herausforderung Daten und Datenquellen}

Eine Hauptaufgabe bei der Umsetzung einer digitalen Lösung zur Brandschutzbedarfsplanung besteht vor allem in der Ermittlung und Auswahl der benötigten Datenquellen. Grundsätzlich ergeben sich die benötigten Geoinformationen für die Brandschutzbedarfsplanung aus der Verwaltungsvorschrift (VV). Aufgrund der Komplexität und Unterschiedlichkeit der angebotenen Daten nimmt die Recherche und Auswahl für die Brandschutzbedarfsplanung nach am besten geeigneten Daten relativ viel Zeit in Anspruch und gestaltete sich in einigen Punkten schwierig. Die größte Herausforderung besteht in der Bewertung der vorhandenen Daten und dem Umgang mit fehlenden Daten.

Zur Recherche wurden die Portale des Landes sowie eigene Erfahrungen und Datenbestände im Rahmen des Infrastrukturknotens der GDI MV genutzt. Bei der anschließenden Gegenüberstellung der Datenbestände und der Identifizierung der Quellen zeigte sich, dass ein Teil der benötigten Datenbestände nicht flächendeckend oder redundant vorliegen. Grundsätzlich sind beim Vergleich die jeweiligen Fragestellungen und notwendigen Analysen zu beachten, um die passende Qualität mit der strukturellen, inhaltlichen und zeitlichen Auflösung zu wählen.

Die Ursache für redundante Datensätze liegt offensichtlich in den unterschiedlichen Zielen der Erzeugung und Verwendung. Die wesentlichen Unterschiede bei redundanten Daten lassen sich wie folgt kategorisieren:

- Verschiedene Objekte

- Geometrische Unterschiede bei gleichen Objekten (Lage und Struktur)

- Zugriffsmöglichkeit Datensatz (Geowebdienst, lokale Datei)

- Aufbau, Inhalte und Attribute des Datenbestands

- Aktualisierungszyklus des Datenbestands (laufend, bei Bedarf)

Diese Sachverhalte sollen an drei Beispielen aufgezeigt werden: 
Flüsse stehen im ATKIS-Datenmodell der Landestopographie sowie als Umweltinformation des Landesamts für Umwelt, Nutzerschutz und Geologie bereit. Im ATKIS-Datenmodell sind offensichtlich mehr Daten, allerdings ist die Struktur sehr kleinteilig und mit wenigen Attributen im Sinne der Brand- und Katastrophenschutzbedarfsplanung. Im Datensatz des LUNG werden die Flüsse als zusammenhängende Objekte geführt, aber auch dort fehlt die wichtige Information nach der Tiefe des Flusses.

Die Schulstandorte werden von den Kreisverwaltungen und vom Statistischen Amt veröffentlicht. Im direkten Vergleich (Themenkarten unter www.geoportalmv.de) sind Unterschiede erkennbar. In den Daten des Statistischen Amts findet sich der aktuelle Stand des Schuljahres inkl. Klassen und Schülerzahlen. In den Daten der Landkreise finden sich allerdings auch geplante und mögliche Standorte; beides ist für die Brandschutzbedarfsplanung relevant.

Die Verwaltungsgrenzen können aktuell sowohl aus den Digitalen Verwaltungsgrenzen (DVG) als auch aus dem Liegenschaftskataster bezogen werden. In der Lage gibt es zum Teil erhebliche Unterschiede und es kann bei der räumlichen Zuordnung eines Fachdatensatzes zu Überschneidungen kommen, wenn nicht einheitlich nur einer der beiden Grenz-Datensätze verwendet wird. Die Daten des DVG wurden mit der notwendigen Genauigkeit der Topographie unter Berücksichtigung gesetzlicher Vorgaben, insbesondere bei den Seegrenzen, erfasst. Die Gemeinden des Liegenschaftskatasters beruhen direkt auf den Flurstücksgrenzen.

Sind Geoinformationen hingegen nicht flächendeckend vorhanden, ergeben sich in der Darstellung der Situation für die Brandschutzbedarfsplanung Lücken, die kompensiert werden müssen. Sofern weitere Auswertungen darauf beruhen, sind diese vorab zu ergänzen. Alternativ können die Planer die Ergebnisse ihrer regionalen Datenerhebung später hinzufügen. Hierzu zählen unter anderem Wasserversorgung und Hydranten, weil dies von verschiedensten lokalen Anbietern bereitgestellt wird.

\section{Ausblick}

Das Modul zur Erstellung der Vorlage steht im GeoPortal.MV für registrierte und freigeschaltete Nutzer bereit. Es bildet die erste Grundlage für eine Datenerhebung und kann direkt oder auch indirekt für die weitere Brandschutzbedarfsplanung verwendet werden. 
Im Sinne der Brandschutzbedarfsplanung gibt es bereits zahlreiche Anhaltspunkte zum weiteren Ausbau. So können weitere Datenquellen einbezogen und ausgewertet werden, die derzeit noch manuell erfasst werden oder auf die noch nicht zugegriffen werden können. Hinzu können weitere Analysen, z.B. zur Erreichbarkeit, automatisiert werden.

Im weiteren Verlauf besteht das Interesse daran, auch die eigentliche Risikoanalyse weiter zu automatisieren, um mehr vergleichbare Ergebnisse zu erhalten. Derzeit wird hierfür in Schleswig-Holstein eine Weblösung getestet, die für diese Aufgabe geeignet sein könnte. Bevor die Lösung jedoch nutzenstiftend eingesetzt werden kann, bedarf es einer soliden Grundlage der Geoinformationen.

Die gewonnenen Erfahrungen im Rahmen der vorgestellten Lösung werden dokumentiert und können für weitere Anwendungsfälle eingesetzt werden. Im Sinne der NGIS werden somit die Daten weiter nachgenutzt und auch die Bereitstellung von notwendigen fehlenden Daten gefördert. 



\title{
Earth Observation Maritime Surveillance System
}

\author{
Sergey Voinov ${ }^{* 1}$, Egbert Schwarz ${ }^{1}$, Detmar Krause ${ }^{1}$, Björn Tings ${ }^{2}$ \\ ${ }^{1}$ German Remote Sensing Data Center (DFD), German Aerospace Center (DLR) \\ ${ }^{2}$ Remote Sensing Technology Institute (IMF), German Aerospace Center (DLR) \\ Kalkhorstweg 53, 17235, Neustrelitz \\ *sergey.voinov@dlr.de
}

\begin{abstract}
Synthetic Aperture Radar (SAR) and medium (MR) and very high (VHR) resolution optical satellite images are valuable sources of information for maritime situational awareness. This paper presents the Earth Observation Maritime Surveillance System (EO-MARISS), an integrated processing framework of remote sensing satellite data developed at DLR's Maritime Safety and Security Lab Neustrelitz to support sea monitoring tasks. The system is currently developed for operational use at DLR's Ground Station Neustrelitz to support service provider like AIRBUS or EUSI and processes image data from different satellite missions received directly at the ground station or provided by a network of ground stations and service providers.
\end{abstract}

Keywords: SAR, Optical, Remote Sensing, Maritime Security, Maritime Surveillance, Near Real Time

\section{Introduction}

Remote sensing data are widely used to enhance maritime surveillance applications. The most frequent use cases of satellite images in this domain are environmental and vessel traffic monitoring. Different kind of complementary sensor types providing unique capabilities for solving these tasks. Since decades Synthetic Aperture Radar (SAR) satellite data has been proven to be effective for ship detection (Tings, et al., 2019) and environmental monitoring, such as oil spill detection, as well as sea state parameter estimation (Schwarz, et al., 2015). The main advantages of SAR sensors are their weather independence and capacity to cover very large areas. For example, the Copernicus Sentinel-1(A/B) satellites may cover up to $250 \mathrm{~km}$ wide area in Interferometric (IW) swatch mode with 5x20 meters of ground sampling distance. Medium resolution (MR) multispectral optical sensors like Copernicus Santinel-2 or Landsat-8 are beneficial 
for environmental monitoring tasks due to their spectral resolution (Voinov, et al., 2018). Moreover MR sensors are capable to cover relatively large areas, sometimes comparable to SAR missions. Very high resolution (VHR) optical satellite images are more suitable for sea traffic monitoring. The level of details of such images is sufficient to detect and classify vessels of different types and sizes (Voinov, et al., 2019). Combinations of all mentioned sensor types in one system would offer great capacities for solving wide range of maritime surveillance tasks.

This paper presents the Earth Observation Maritime Surveillance System (EOMARISS), an integrated processing framework of remote sensing satellite data to support sea monitoring tasks in fully automated or semi-automated way (depending on scenario).

Algorithms for processing of optical data as well as framework design and integration at DLR's ground station Neustrelitz is carried out by the Maritime Security Lab in Neustrelitz, part of the DLR's German Remote Sensing Data Center (DFD). The Maritime Security Lab in Bremen, part of DLR's Remote Sensing Technology Institute (IMF) is responsible for development of SAR processing algorithms.

\section{System Overview}

The current EO-MARISS implementation supports following SAR satellite sensors: TerraSAR-X, TanDEM-X, Radarsat-2 and the Copernicus mission Senti-nel-1; MR optical satellite sensors Landsat-8 and Copernicus Sentinel-2; and VHR optical satellite sensors GeoEye-1, WorldView-[1-3] and Deimos-2. Furthermore, it will be upgraded to support the upcoming WorldView-Legion con-stellation after its launch. Multiple framework instances are deployed in a cluster of virtual machines to support different missions simultaneously.

Services based on TerraSAR-X and TanDEM-X satellites for the commercial market are available via AIRBUS DS. Services based on the VHR optical sensors are available via the European Space Imaging Company (EUSI), key distributor of VHR satellite imagery in Europe.

The EO-MARISS overall architecture is shown on Figure 1 which is represented as five-tier system: 1 . data; 2. processing environment; 3 . processing chain; 4. product dissemination; and 5 . users. 


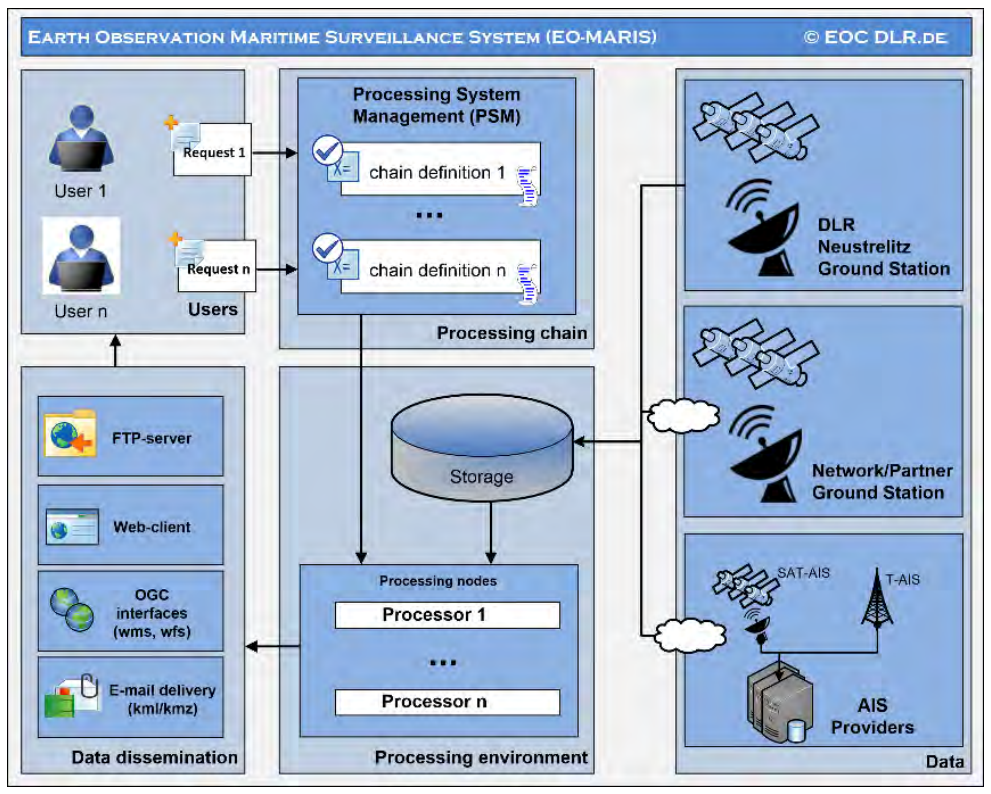

Figure 1: EO-MARISS Architecture

If the data received by the local ground station, the first step is generation of level 1 (L1) image product, which is sensor-specific. This operation converts raw sensor data into image representation form with applying radiometric calibrations and extraction of geolocation information. If reception is handled by the partners (data providers) and not by the local ground station, a dedicated ingestion processor retrieves the L1 product via the network.

The preferred data source and requested value-adding product are the main parameters defined by the user in the request order. Based on these parameters, the system generates a special runtime workflow.

The entire processing chain is controlled by the Processing System Management (PSM), the software developed jointly by the DFD and Werum Software \& Systems AG (Boettcher, et al., 2001). The PSM is orchestrating the hardware resources and the processing workflow. Depending on the scenario it may run different processors in parallel or in a step-by-step sequence. The typical processing sequence starts with the image preprocessing step followed by a set of 
information extraction and value-adding processors, which are operated in a fully automated or semi-automated way.

For SAR satellites, generation of following product types is supported in a fully automated way: vessel detection and fusion with AIS, sea state parameter estimation (wind and wave heights) and sea ice classification.

For optical sensors vessel detection product is derived in automated mode. Quality control takes place before the final product is delivered to the user. Other request types such as oil spill, activity and feature detection require operator supervision. For those scenarios interactive map-client application with GIS functionalities has been developed and embedded in the processing chain.

The last step in the sequence is product dissemination. In order to fulfill the delivery requirements of different user groups a number of dissemination options have been developed. This includes: OGC-standardized raster and vector file formats; file formats specified by the European Maritime Safety Agency (EMSA); web-based OGC interfaces (wms and wfs); special web client (shown in Figure 2) which requires no special software and skills from the end user.
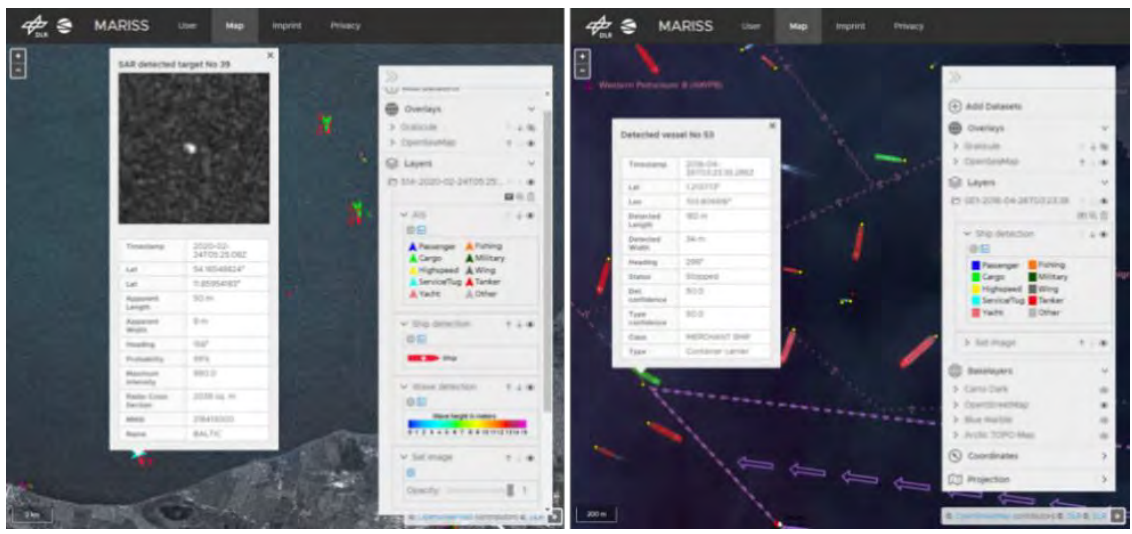

Figure 2: EO-MARISS web-mapping client. On the left side shown example of vessel detection product based on SAR image and on the right - Opticalbased 


\section{Summary}

In this paper, the Earth Observation Maritime Surveillance System (EOMARISS) has been presented. The system is implemented with high degree of automatization and designed to be near real time (NRT)-oriented. It supports both, SAR and optical satellite image processing and offers variety of valueadding product types. The presented system is deployed in a cluster of virtual machines and can handle several requests simultaneously by providing distributed operator workplaces in parallel. Further development will focus on integration of new sensor types and development of new modules to support a larger variety of maritime security applications.

\section{Bibliography}

Boettcher, M., Reißig, R., Mikusch, E. \& Reck, C., 2001. Processing Management Tools for Earth Observation Products at DLR-DFD. Nice, European Space Agency.

Schwarz, E., Krause, D., Daedelow, H. \& Voinov, S., 2015. Near Real Time Applications For Maritime Situational Awareness. Rostock, s.n.

Tings, B., Bentes da Silva, C. A., Velotto, D. \& Voinov, S., 2019. Modelling Ship Detectability Depending On TerraSAR-X-derived Metocean Parameters. CEAS Space Journal, Volume 11, pp. 81-94.

Voinov, S., Heymann, F., Bill, R. \& Schwarz, E., 2019. Multiclass Vessel Detection From High Resolution Optical Satellite Images Based On Deep Neural Networks. Yokohama, Japan, IEEE, pp. 166-169.

Voinov, S., Schwarz, E., Krause, D. \& Berg, M., 2018. Processing framework to support maritime surveillance applications based on optical remote sensing images. Paphos, Cyprus, SPIE. 

Geoinformation als Ressource 



\title{
PSI-Richtlinie ante portas - Geodaten als ,hochwertige Datensätze ${ }^{66}$
}

\author{
Falk Zscheile \\ Kramp, Selling und Partner Rechtsanwälte \\ Neuer Markt 12, 18055 Rostock \\ zscheile@kramp.de
}

\begin{abstract}
Der europäische Gesetzgeber hat die PSI-Richtlinie (Re-use of Public Sector Information) nach dem Inkrafttreten 2003 und Änderungen im Jahr 2013 bereits ein weiteres Mal novelliert und als neue Richtlinie erlassen (RL 2019/1024/EU). Die Änderungsgeschichte der Richtlinie zeigt den unbedingten Willen zu Open Data bei Verwaltungsinformationen allgemein. Die aktuelle Fassung PSI-Richtlinie schafft erstmals die Kategorie der hochwertigen Datensätze, Art. 13, 14 PSI-RL (2019). Für hochwertige Datensätze fordert Art. 6 Abs. 6 PSI-RL (2019) künftig unter anderem Kostenfreiheit. In der Anlage 1 zu Art. 13 Abs. 1 PSI-RL (2019) wird der „Georaum“ als Kategorie für solch hochwertige Datensätze aufgeführt.
\end{abstract}

\section{$1 \quad$ Einleitung}

Der europäische Gesetzgeber verfolgt seit nunmehr fast 20 Jahren das Ziel, die Verwaltungsinformationen von Behörden der Mitgliedsstaaten für den Binnenmarkt verfügbar zu machen. Die ursprüngliche Richtlinie über die Weiterverwendung von Informationen des öffentlichen Sektors (Dierective on the reuse of Public Sector Information, PSI-RL, 2003/98/EG) wurde seitdem bereits zwei Mal überarbeitet. Die Änderung aus dem Jahre 2013 (Richtlinie zur Änderung der Richtlinie 2003/98/EG, RL 2013/37/EU) begnügte sich dabei noch mit der bloßen Änderung an der Ausgangsrichtlinie. Mit der PSI-Richtlinie aus dem Jahr 2019 liegt nunmehr eine vollständig überarbeitete und neu formulierte Fassung vor (RL 2019/1024/EU). In der neuesten Fassung wird erstmals die Kategorie der „hochwertigen Datensätze“ eingeführt und mit entsprechenden besonderen rechtlichen Regeln versehen. Besonders bemerkenswert ist dabei, dass Geodaten ausdrücklich als hochwertige Datensätze genannt werden. Damit existiert neben der INSPIRE-Richtlinie nunmehr eine weitere rechtliche Rege- 
lung, die sich ausdrücklich mit der Bereitstellung von Geodaten durch die öffentliche Verwaltung beschäftigt.

Die ursprünglichen Ziele der PSI-Richtlinie nach einer Stärkung des Binnenmarktes durch Zweitverwertung von Verwaltungsinformationen sind dabei unverändert geblieben. Mit der Entwicklung hin zu einer Informationsgesellschaft mit Daten als Treibstoff hat die PSI-Richtlinie den Fokus immer stärker auf Kostenfreiheit, Verfügbarkeit und Open (Government) Data gelegt. Der Stärkung einer datengestützten Wirtschaft und Gesellschaft wird damit klare Priorität gegenüber einem etwaigen Refinanzierungsinteresse des Staates (Weiterverkauf der Daten) eingeräumt.

Gegenstand der Regelung der PSI-Richtlinie ist dabei ausschließlich die Frage der Nutzung von Verwaltungsinformationen, nicht aber der Zugang zu Verwaltungsinformationen. $\mathrm{Ob}$ eine Verwaltung überhaupt ihre Informationen freigeben muss, ist kein Regelungsgegenstand der PSI-Richtlinie. Der Zugang zu Verwaltungsinformationen muss sich also zwingend aus einer anderen gesetzlichen Grundlage ergeben. Die PSI-Richtlinie regelt die Weiterverwendung, aber nicht den Zugang zu Verwaltungsinformationen. Demgegenüber behandelt die INSPIRE-Richtlinie sowohl Fragen des Zugangs zu Geodaten als auch deren Nutzung/Weiterverwendung.

Wie bei allen europäischen Richtlinien gilt auch hier, dass die Richtlinie selbst keine unmittelbare rechtliche Wirkung entfaltet, sondern durch eine nationale Regelung in das jeweilige Recht des Mitgliedstaats überführt werden muss. Die aktuelle PSI-Richtlinie ist bis zum 17. Juli 2021 in nationales Recht zu überführen Art. 17 Abs. 1 PSI-RL (2019).

\section{Hochwertige Datensätze}

Bei den bereits eingangs erwähnten „hochwertigen Datensätzen“ handelt es sich ganz allgemein um Dokumente, die mit wichtigen sozioökonomischen Vorteilen verbunden und für die Wirtschaft und die Gesellschaft von besonders hohem Wert sind, vgl. Art. 2 Nr. 10 PSI-RL und Art. 14 Abs. 2 PSI-RL (2019). Dabei darf man sich vom Begriff „Dokument“ nicht irritieren lassen. Anders als im allgemeinen Sprachgebrauch ist mit Dokument jeder Inhalt oder Teil davon unabhängig von der Form des Datenträgers gemeint, Art. 2 Nr. 6 PSI-RL (2019). 
Die Vorteile, die sich aus „hochwertigen Datensätzen“ ergeben, können dabei ganz unterschiedlicher Natur sein. Ein Aspekt ist die Eignung zur Schaffung von Mehrwertdiensten, ein anderer die Zahl der potenziellen Nutznießer solcher auf öffentlichen Daten aufbauender Mehrwertdienste. Auch die Schaffung neuer hochwertiger und menschenwürdiger Arbeitsplätze wird als ein Aspekt angeführt.

Soweit es sich um einen „hochwertigen Datensatz“ handelt, werden die Möglichkeiten zu dessen Nutzung sehr klar herausgearbeitet: Diese Datensätze sollen mit minimalen rechtlichen Einschränkungen kostenlos zur Weiterverwendung zugänglich gemacht werden.

Dabei liegt ein besonderer Schwerpunkt auf den technischen Anforderungen der einfachen Verfügbarkeit und Verarbeitung solcher Informationen: Die Informationen müssen maschinenlesbar, über eine API und gegebenenfalls als Massendownload verfügbar sein, Art. 14 Abs. 1 PSI-RL (2019). Die Details können durch die Europäische Kommission über entsprechende Durchführungsakte geregelt werden.

Mit den „minimalen rechtlichen Einschränkungen“ werden insbesondere die rechtlichen Anforderungen in den Fokus genommen. Verwaltungsinformationen können durch Ausschließlichkeitsrechte (z. B. Urheberrecht, Datenbankherstellerrecht) geschützt sein. Die Nutzung ist dann nur erlaubt, wenn ein entsprechendes Nutzungsrecht (Lizenz) eingeräumt wurde. Die PSI-Richtlinie verlangt insoweit, dass die Veröffentlichung und Weiterverwendung von „hochwertigen Datensätzen“ mit offenen Standardlizenzen vereinbar sein müssen.

Die „hochwertigen Datensätze“ müssen zudem kostenlos zur Verfügung stehen, Art. 14 Abs. 1 lit. a) PSI-RL (2019) und Art. 6 Abs. 6 lit. a) PSI-RL (2019). Dies ergibt sich zwar implizit bereits aus der Forderung nach einer offenen Lizenz, wird aber zur Sicherheit neben der Frage der Lizenzierung thematisiert. Aus der expliziten Erwähnung der Kostenfreiheit ergibt sich mit Blick auf Art. 6 Abs. 1 PSI-RL (2019), der ebenfalls die Kostenfreiheit von Verwaltungsinformationen regelt, dass die Kosten der Reproduktion, Bereitstellung und Verbreitung sowie der Anonymisierung von Daten nicht auf die Nutzer umgelegt werden dürfen. Dies ist nur für Daten zulässig, die nicht als „,hochwertige Datensätze" geführt werden.

Die Forderung der PSI-Richtlinie nach einer kostenfreien Bereitstellung von „hochwertigen Datensätzen“ wirft mit Blick auf Geodaten („Georaum“), die in Anlage 1 zu Art. 13 Abs. 1 PSI-RL (2019) als „hochwertige Datensätze“ genannt sind, die Frage nach dem Verhältnis zur INSPIRE-Richtlinie auf. 
Die INSPIRE-Richtlinie lässt Gebühren für Geodaten dem Grunde nach zu. Ausnahmen bilden hier unter bestimmten Voraussetzungen beispielsweise die Darstellungsdienste, Art. 14 Abs. 1 INSPIRE-RL. Die Qualifizierung von Geodaten als „hochwertige Datensätze“ im Sinne der PSI-Richtlinie beseitigt die Möglichkeit, auf Basis der INSPIRE-Richtlinie Gebühren für Geodaten zu erheben, weitgehend, ohne dass hierfür die INSPIRE-Richtlinie geändert würde. Die INSPIRE-Richtlinie selbst geht in Erwägungsgrund 8 noch davon aus, dass sich ihre Ziele und die Ziele der PSI-Richtlinie ergänzen. Davon wird man künftig nicht mehr ausgehen dürfen. Die PSI-Richtlinie (2019) spricht in ihrem Erwägungsgrund 4 davon, dass sich die wesentlichen Änderungen am Rechtstext der PSI-Richtlinie unter anderem auf die Inanspruchnahme von Ausnahmen vom Grundsatz der Gebührenbeschränkung auf die Grenzkosten und das Verhältnis dieser Richtlinie unter anderem zur Richtlinie 2007/2/EG (INSPIRE-RL) konzentrieren. Hierin kann man den Willen des europäischen Gesetzgebers erblicken, die Möglichkeiten von Gebühren, welche die INSPIRE-Richtlinie zulässt, zu beschränken und Kostenfreiheit für Geodaten durchzusetzen.

In Art. 14 Abs. 3 bis Abs. 4 PSI-RL (2019) sind für bestimmte öffentliche Institutionen Ausnahmen von den besonderen Anforderungen für „hochwertige Datensätze" vorgesehen. Hierauf können sich auch künftig geodatenhaltende Stellen berufen. Eine Berufung auf die „Gebührenregeln“ der INSPIRERichtlinie dürfte hingegen künftig nicht mehr möglich sein. Die nationalen Gesetzgeber werden insoweit ihre Gebührenregeln für Geodaten überprüfen und gegebenenfalls anpassen müssen.

\section{$3 \quad$ Nationale Umsetzung}

Wie bereits eingangs erwähnt, entfalten europarechtliche Richtlinien keine unmittelbare Wirkung im nationalen Recht, sondern bedürfen der Überführung in das Recht des jeweiligen Mitgliedstaats. Für die gesetzgeberischen Zuständigkeiten innerhalb des jeweiligen Mitgliedstaats interessiert sich der europäische Gesetzgeber dabei nicht.

In Deutschland wird die PSI-Richtlinie durch den Bund als Gesetzgeber in nationales Recht überführt. Die Umsetzung ist mit dem „Gesetz über die Weiterverwendung von Informationen öffentlicher Stellen“" (IWG) erfolgt. Der Bund kann sich hierbei auf seine Gesetzgebungskompetenz für das Recht der Wirtschaft, Art. 70 Abs. 1, 74 Abs. 1 Nr. 11 GG, berufen. Die Bundesländer sind, soweit diese Gesetzgebungskompetenz reicht, insoweit nicht zu einer eigenen oder parallelen Umsetzung befugt. 
Geoinformationen fallen hingegen aufgrund fehlender abweichender Regelungen grundsätzlich in den Bereich der Gesetzgebungskompetenz der Länder, Art. 70 Abs. 1 GG. Dementsprechend mussten die Regelungen der INSPIRERichtlinie sowohl auf Bundesebene als auch in den 16 Bundesländern durch eigenes Gesetz umgesetzt werden. Dabei bildeten die Gebühren im Zusammenhang mit Geoinformationen einen Annex zur Zuständigkeit der Länder für ihre Geoinformationen.

Der Blick auf die PSI-Richtlinie mit dem Ziel der Stärkung des Binnenmarktes zeigt aber, dass sich Gebührenregelungen für Verwaltungsinformationen durchaus im Spannungsfeld zwischen der Gesetzgebungskompetenz des Bundes für das Recht der Wirtschaft und der Zuständigkeit der Länder bewegt.

Bereits in seiner jetzigen Fassung, also ohne Berücksichtigung der PSIRichtlinie 2019, enthält $\S 1$ Abs. 2 Nr. 8 IWG als Bundesgesetz eine Regelung, welche die Gebührenfreiheit für Geodaten der Länder fordert. Danach ist das Gesetz über die Weiterverwendung von Informationen öffentlicher Stellen nur dann nicht auf Geodaten der Länder anwendbar, wenn eine Weiterverwendung nach Landesrecht uneingeschränkt möglich ist. Ob diese Regelung mit Blick auf die Verteilung der Gesetzgebungskompetenzen zwischen Bund und Ländern zulässig ist, ist bisher ungeklärt. Mit Blick auf die Vorgaben der PSI-Richtlinie werden künftig alle Bundesländer ihre Gebührenregelungen im Hinblick auf Geodaten überdenken und anpassen müssen, soweit die Abgabe von Geodaten nicht schon jetzt im Rahmen einer Open Betastrategie erfolgt.

\section{$4 \quad$ Zusammenfassung und Ausblick}

Der europäische Gesetzgeber hat mit der überarbeiteten PSI-Richtlinie seinen unbedingten Willen zu Open Government Data manifestiert. Durch die Kategorie „hochwertige Datensätze“ hat der europäische Gesetzgeber künftig die Möglichkeit, bestimmte Arten von Informationen ohne Wenn und Aber dem Anwendungsbereich der PSI-Richtlinie zuzuweisen. Für Geodaten bedeutet das, dass die in der INSPIRE-Richtlinie rudimentär vorhandenen Regelungen zur Lizenzierung und zu Gebühren für Geodaten künftig durch die Regelungen der PSI-Richtlinie verdrängt werden. Die nationalen Regelungen müssen entsprechend angepasst werden. Der Wirtschaft werden künftig, nach Umsetzung der PSI-Richtlinie in das nationale Recht, flächendeckend geographische Informationen der Landesverwaltungen als Open Data zur Verfügung stehen. 



\title{
Kommunale Nachnutzungsmöglichkeiten von Daten der Landesvermessung Mecklenburg-Vorpommern
}

\author{
Jürgen Schulz \\ Hansa Luftbild AG \\ schulz@hansaluftbild.de
}

\begin{abstract}
Die Landesvermessung Mecklenburg-Vorpommerns (MV) lässt seit vielen Jahren Befliegungen durchführen, um aktuelle Luftbilder und Laserdaten zur Verfügung zu haben. Die Daten werden aufbereitet ebenfalls den Kommunen zur Verfügung gestellt. Die gute geometrische Auflösung der Daten $(10 \mathrm{~cm})$, die hohe Bildüberlappung (in den 5 größten Städten sogar $80 \% / 60 \%$ ) und die zusätzliche Verfügbarkeit von Laserdaten eröffnen den Kommunen in MV die Möglichkeit, weitere Produkte aus diesen Daten abzuleiten. Das könnten in erster Linie kommunale Kataster sei, die das Anlagevermögen der Kommune erfassen oder die praktische Verwaltungsarbeit digitalisieren und damit effektivieren. Als Beispiele seien genannt: Straßenkataster, Versiegelungskataster und Grünflächenkataster. Solche Kataster können durch externe spezialisierte Dienstleister aufgebaut oder aktualisiert werden. Es gibt aber weit mehr Anwendungsmöglichkeiten - unter anderem die Nutzung als Basisdaten für den Schutz vor Starkregenereignissen (urbane Sturzfluten, urban flooding). Diese immer häufiger und meist plötzlich auftretenden Ereignisse sind ein Ergebnis des Klimawandels und können Menschenleben gefährden. Hierbei geht es darum, passende Geoinformationen für eine Schadenvorsorge oder zumindest Schadenbegrenzung bereitzustellen. Damit können sich die -zur Vorsorge gesetzlich verpflichteten - Kommunen auf derartige Ereignisse vorbereiten. Von A wie Abwassergebührensplitting bis $Z$ wie Zustandserfassung der Straßen - die aktuellen Geodaten der Landesvermessung sind eine wertvolle Quelle zur Ableitung kommunaler Daten. Hansa Luftbild ist nicht nur Dienstleister der Landesvermessung bei den Befliegungen, sondern bietet ebenfalls die ,Veredlung“ dieser Geodaten hin zu echten Geoinformationen an, sei es in Form der o. g. kommunalen Kataster oder durch den Aufbau von WebGIS inklusive Datenhosting.
\end{abstract}




\section{$1 \quad$ Einleitung}

Hansa Luftbild führt - wie der Name es vermuten lässt - Befliegungen durch, und das schon seit sehr langer Zeit. Unsere wichtigsten Kunden sind deutsche Landesvermessungsverwaltungen, deutsche Kommunen, Umweltverwaltungen sowie die Energiewirtschaft. Insbesondere das Interesse der Kommunen hat in letzter Zeit deutlich zugenommen. Dabei geht es zunächst um die reine Befliegung und die Herstellung von Digitalen Orthophotos (DOP) bzw. TrueDOP. Auffallend ist dabei der Trend nach immer besserer geometrischer Auflösung Pixelgrößen von $10 \mathrm{~cm}$ sind ein Minimum und bewegen sich mehr und mehr in Richtung $5 \mathrm{~cm}$. Im vergangenen Jahr haben wir die Stadt Dachau mit einer Pixelgröße von $3 \mathrm{~cm}$ beflogen. Es handelt sich auch keinesfalls nur um die großen Städte. Beispielsweise haben wir aktuell derartige Anfragen u. a. von Gemeinden wie Heidenau, Lotte, Plessa, Biberach, Nordhorn und Bietigheim-Bissingen - Namen, die in MV sicher eher unbekannt sind.

Es kommen also Anfragen und Ausschreibungen aus allen Bundesländern außer aus MV.

Das ist natürlich begründet in den bemerkenswerten Aktivitäten der Landesvermessungsverwaltung Mecklenburg-Vorpommern. Sie versorgt die Landkreise und Städte in einem festgelegten Turnus mit Bild- und Laserdaten. Allerdings sollten Luftbilder nicht zum Selbstzweck, sondern immer auch zur Herstellung abgeleiteter Produkte dienen. In einer Kommune wären das neben dem DOP als Basis des städtischen GIS insbesondere die Ableitung kommunaler Kataster unter Verwendung von Luftbildern. Grünflächenkataster, Versiegelungskataster, Straßenkataster, Baumkataster, Friedhofskataster ... - das ist zugegeben alles nicht neu. Die Frage stellt sich aber:

Nutzen die Kommunen in MV die Daten der Landesvermessung in diesem Sinne?

\section{$2 \quad$ Landesvermessung}

MV ist kein Open-Data-Land. Geodaten kosten also etwas; die Höhe der Kosten ist in einer Gebührenverordnung festgelegt. Das wird so lange so bleiben, bis sich das Land im Sommer 2023 endgültig der Europäischen Richtlinie beugen muss. Im Gegensatz zu Bundesländern wie NRW oder Thüringen ist somit in MV der Erwerb von Luftbildern oder Laserdaten kostenpflichtig. 
Aber natürlich gibt es Ausnahmen bzw. Befreiungstatbestände: Für Kommunen sind die Geodaten frei, aber insbesondere Daten mit hoher geometrischer Auflösung $(10 \mathrm{~cm})$ werden nur beim Vorbringen eines „berechtigten Interesses“ herausgegeben. Dazu zählt zum Beispiel die Verwendung der Geodaten für die Führung von Fachkatastern, was sich dann aber ausschließlich im internen Bereich der Kommune abspielen sollte. Trotz dieser Einschränkungen aus datenschutzrechtlichen Gründen können die Geodaten aber sehr komfortabel über das Geodatenportal des Landes www.geoportal-mv.de recherchiert und bestellt werden.

Das Amt für Geoinformation, Vermessung und Katasterwesen hat ein Bildflugprogramm 2018-2022 aufgestellt, mit dem die Landesfläche in einem Zyklus von 5 Jahren sowohl mit einer Frühjahrs- als auch mit einer Sommerbefliegung abgedeckt wird.

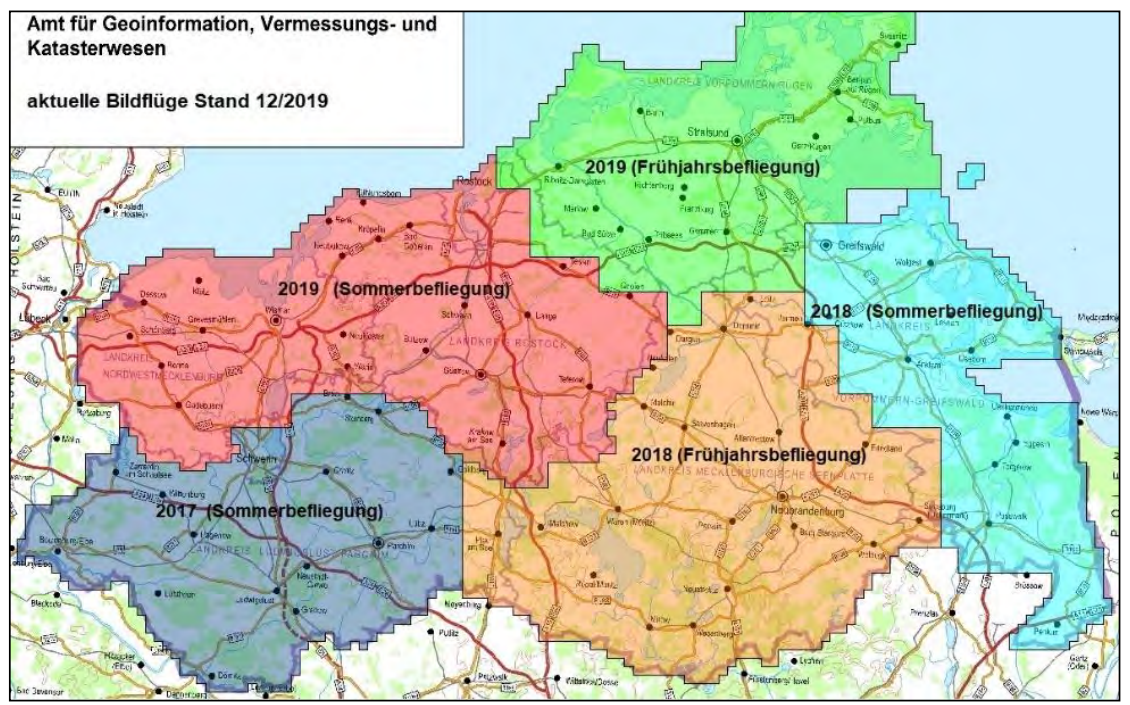

Abbildung 1: Befliegungen des AfGVK 2017-2019 (aus: Geoportal MV)

Da Hansa Luftbild selbst an diesen Befliegungen teilnimmt, sind die technischen Spezifikationen gut bekannt: Die Bodenauflösung liegt bei $10 \mathrm{~cm}$ und die Überlappungen bei $80 \% / 40 \%$, für die Städte Schwerin, Wismar, Rostock, Stralsund und Neubrandenburg bei $80 \% / 60 \%$. 
Die Planung zeigt auch, dass jeder Landkreis - und somit die darin befindlichen Kommunen - alle 5 Jahre in einem Frühjahr beflogen wird. Das ist wichtig zu betonen, weil die nachfolgend beschriebenen Kataster wesentlich auf der Auswertung von Luftbildern der laubfreien Jahreszeit basieren.

\section{$3 \quad$ Kommunale Fachkataster}

Kommunale Verwaltungen sind mit vielfältigen Aktivitäten beschäftigt, um das Leben der Bürger im kommunalen Umfeld zu organisieren. Wasser und Abwasser, Straßenschäden, sichere Schulwege, Pflege von Grünanlagen ... - die Liste der Dinge ist lang und heterogen, um die sich die Verwaltung zu kümmern hat. Geoinformationssysteme (GIS) mit verschiedenen Fachschalen sind seit vielen Jahren zum unentbehrlichen Hilfsmittel geworden. Sie werden oftmals als kommunale Fachkataster bezeichnet, weil sie als Bestandteil des kommunalen GIS aber auch separat auftreten.

\section{Grünflächenkataster}

Kommunen sind verpflichtet, ihre Grünflächen zu pflegen und verkehrssicher zu halten. Dafür ist zunächst die Dokumentation der Grünflächen im öffentlichen Raum erforderlich, auf deren Basis dann die Planung und die Durchführung von Pflegemaßnahmen erfolgt. Im Idealfall gibt es für den Außendienst eine mobile Arbeitsvariante.

Ein luftbildgestütztes Grünflächenkataster basiert auf der Auswertung von Digitalen Orthophotos (DOP) bzw. Original-Luftbildern. $\mathrm{Zu}$ den erforderlichen Datengrundlagen zählen neben den Luftbildern die Katasterdaten (ALKIS), ein Verzeichnis der Grünanlagen und ein Objektkatalog. Mittels der Katasterdaten und dem Verzeichnis der Grünanlagen wird das Untersuchungsgebiet festgelegt; also diejenigen Grünflächen und Flurstücke, innerhalb derer die Datenerfassung erfolgen soll.

Die Bestandserfassung für ein Grünflächenkataster basiert auf einem Objektkatalog. Er definiert diejenigen Flächen-, Punkt- und Linienelemente, die entsprechend der Anforderungen seitens des Auftraggebers zu erfassen sind. Die Vermessung und Auswertung der Grünflächen anhand von Luftbildern führt zu schnellen und geometrisch guten Ergebnissen. In Abhängigkeit vom Objektkatalog und den Anforderungen des Auftraggebers gibt es aber meist Objekte bzw. Sachdaten, die über einen Feldvergleich zu erheben bzw. zu verifizieren sind. Der Feldvergleich überprüft die Ergebnisse der Luftbildvermessung und ergänzt 
Informationen, die über die reine Luftbildauswertung nicht geführt werden können.

Nach Luftbildauswertung und Feldvergleich werden die Daten für das Zielsystem des Auftraggebers aufbereitet. Das Zielsystem (GIS, spezielle Software beim Auftraggeber etc.) muss frühzeitig bekannt sein, damit die Anforderungen des Systems bei der Datenerfassung und -aufbereitung berücksichtigt werden können.

\section{Straßenkataster}

Die Verkehrsinfrastruktur gehört zu den wichtigsten Vermögenswerten einer Kommune. Zu deren Dokumentation und weiteren Planung ist der Aufbau eines professionellen Straßenkatasters unerlässlich. Ohne ein solches Kataster kann weder der Bestand noch der Zustand der Straßen richtig bewertet werden - nicht quantitativ und qualitativ und auch nicht monetär.

Die Methodik der Erarbeitung eines luftbildgestützten Straßen-Bestandskatasters ist sehr ähnlich der Methodik für ein Grünflächenkataster bzw. der für alle kommunalen Kataster. Das Zielobjekt ist natürlich jetzt der Straßenraum mit all seinen vielfältigen Objekten (Belag, Verkehrszeichen, Markierungen ...). Hierfür ist neben den Katasterdaten ein sehr detaillierter Katalog der zu erfassenden Elemente notwendig.

Grundsätzlich erfolgt die Erfassung und Aufbereitung nach dem sog. Realflächenmodell (in Anlehnung an das FGSV-Arbeitspapier Nr. 9 zum Management der Straßenerhaltung). Das dort beschriebene Modell stellt eine reale Vor-OrtSituation dar. Nach der Bestandsdatenerfassung werden die Daten für das Zielsystem (GIS, spezielle Software beim Auftraggeber etc.) des Auftraggebers aufbereitet. Ein Knoten-Kanten-Modell des Kunden ist bei den Arbeiten hilfreich, aber nicht unbedingt notwendig.

Ein Straßenkataster des Bestandes kann als Basis zur Erweiterung zur Erfassung und Bewertung des Zustandes dienen.

\section{Weitere kommunale Kataster}

Das Vorhandensein aktueller Luftbilder erlaubt die Erarbeitung weiterer kommunaler Kataster, wie z. B. Baumkataster, Friedhofskataster, Spielplatzkataster oder Baulückenkataster. Da es sich um Kartierungen handelt, bei denen den Objekten ein Attribut zugeordnet werden muss, ist der Automatisierungsanteil nach wie vor gering. 


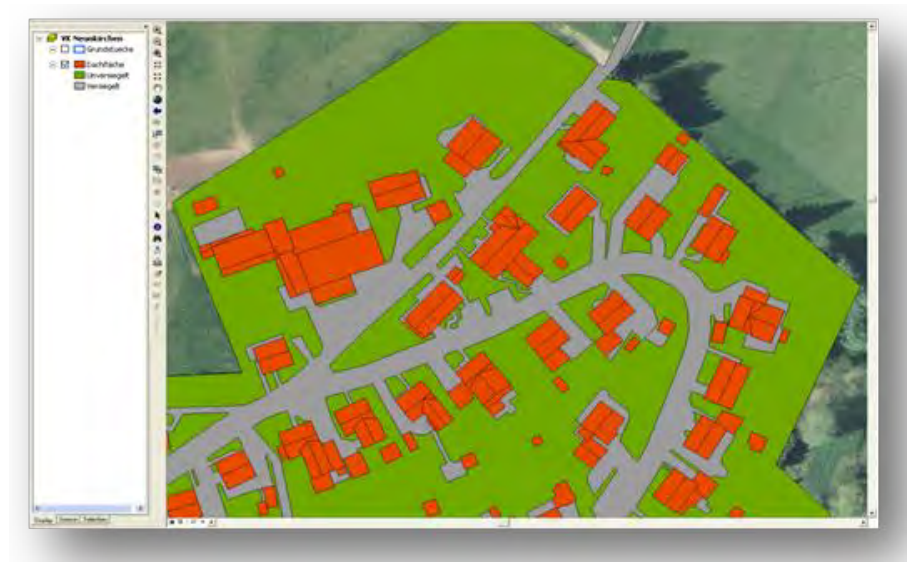

\section{Abbildung 2: Versiegelungskartierung}

\section{Vorbereitung auf Starkregenereignisse}

Der Klimawandel bringt es mit sich, dass wir immer häufiger kurze extreme Witterungsereignisse haben - unter anderem Starkregenereignisse oder urbane Sturzfluten. Voll gelaufene Keller und Tiefgaragen sind sehr ärgerlich, teilweise gibt es aber auch Gefahr für Leib und Leben. Eine nachträgliche Schadenkartierung ist mittels Satellit, Flugzeug oder Drohne natürlich machbar. Der bessere Weg ist aber, die passenden Geoinformationen für eine Schadensvorsorge oder zumindest Schadensbegrenzung bereitzustellen. Damit können sich die Kommunen auf derartige Ereignisse vorbereiten.

In Deutschland gibt es bereits die ersten Kommunen, die Geodaten für den Schutz vor urbanen Sturzfluten verwenden. Nicht nur, weil sie zur Vorsorge gesetzlich verpflichtet sind, sondern auch, weil viele Kommunen bereits negative Erfahrungen machen mussten.

Die Kernfragen bei diesem Problem heißen „Wohin fließt das Wasser?“ und „Wo richtet es Schäden an?". Dazu benötigt man eine örtliche, detaillierte modellgestützte Untersuchung. Eine solche Simulation erfordert gute Eingangsdaten. Einige dieser Daten sollten bei jeder Kommune vorhanden sein (Gebäudebestand, Kanaldaten etc.). Andere Daten sind nicht oder in nicht ausreichender Qualität vorhanden (Höhenmodell, Bruchkanten etc.). Luftbilder sind für die Berechnung guter Digitaler Geländemodelle (DGM) und Digitaler Höhenmodelle eine ideale Basis. Darüber hinaus stellt die Landesvermessung aber auch diese Produkte auf der Basis von Laserscan-Befliegungen bereit. 
Daher ist es erforderlich, präzise Daten der möglichen Fließwege des Wassers bereitzustellen (d. h. auch die Höhe der Bordsteinkanten, die Lokalität jedes Gullys, die Erfassung jedes Hindernisses).

Neben den Daten über die städtische Kanalisation sind dies alles wichtige Eingangsinformationen für eine hydraulische Simulation. Dafür gibt es zahlreiche kommerzielle Programme und es gibt spezialisierte Unternehmen, mit denen Hansa Luftbild kooperiert.

Schlussendlich geht es um die Herstellung von Gefahrenkarten oder Risikokarten eines Stadtgebietes, welche lebenswichtige Informationen für die Stadtplanung, die Stadtentwässerung, den Katastrophenschutz und die Öffentlichkeit bereitstellen. Die Daten können für die Vorsorge vor dem Starkregen genutzt werden, z. B. für

- $\quad$ Anlegen von Sickergräben und von Regenrückhaltebecken

- $\quad$ Entsiegelung von Flächen oder Ersatz durch wasserdurchlässige Flächenbeläge

- $\quad$ Errichtung von Hindernissen (Mauern), um Fließwasser in Richtungen zu zwingen

- Dachbegrünungen

- $\quad$ Freihaltung von Flächen zur natürlichen Versickerung, Grünanlagen anlegen

Die Gefahren von urbanen Sturzfluten sind real und man muss den Schutz vor ihnen seriös vorbereiten. Gute Simulationsprogramme verlangen auch gute Geodaten als Basis.

\section{Zusammenfassung und Ausblick}

Eine kurze, nicht-repräsentative Recherche ergab, dass Kommunen in MV die Möglichkeiten der Daten der Landesvermessung nur in geringem Maße nutzen. Natürlich gibt es kommunale GIS bzw. Kataster; aber der mittels Luftbildern erzielbare Mehrwert ist selten sichtbar. Diese Chancen sollten die Kommunen (ggf. mit Unterstützung von Dienstleistern) besser nutzen. Ein relativ einfacher Beginn wäre z. B. Aktualisierung vorhandener Kataster mithilfe der Daten aus der neuesten Befliegung. 



\title{
Umfrage zur Situation der Wertstoffhöfe in der Hanse- und Universitätsstadt Rostock
}

\author{
Ferdinand Vettermann ${ }^{1}$, Samer Nastah ${ }^{1}$, Laurine Larsen ${ }^{2}$, Ralf Bill ${ }^{1}$ \\ ${ }^{1}$ Universität Rostock, ${ }^{2} \mathrm{BN}-U m w e l t \mathrm{GmbH}$ \\ \{ferdinand.vettermann|samer.nastah|ralf.bill\}@uni-rostock.de \\ 1.larsen@bn-umwelt.de
}

\begin{abstract}
Ein wesentliches Element im Rahmen des Projektes „Prospektive synergistische Planung von Entwicklungsoptionen in Regiopolen am Beispiel des Stadt-Umland-Raumes Rostock“ (Prosper-Ro) ist die Analyse der Kreislaufwirtschaft und damit der Wertstoffhöfe. Diese wurden im Rahmen dieser Untersuchung sowohl mittels GIS-basierter Methoden als auch mittels einer Umfrage analysiert. Es konnte gezeigt werden, dass das Gros der Bevölkerung zufrieden mit den Höfen Rostocks ist. Auch die Erreichbarkeiten sind als gut zu bezeichnen, obgleich sich diese v. a. im Landkreis verbessern ließen. In der Stadt hingegen ergibt sich eher ein Überlastungsproblem. In diesem Kontext ist allerdings gerade hinsichtlich der Planung die Entwicklung genauerer Bedarfsparameter notwendig.
\end{abstract}

\section{$1 \quad$ Einleitung und Stand der Forschung}

Im Rahmen des durch das Bundesministerium für Bildung und Forschung (BMBF) geförderten Projektes „Prospektive synergistische Planung von Entwicklungsoptionen in Regiopolen am Beispiel des Stadt-Umland-Raumes Rostock" (Prosper-Ro) (Tränckner, 2017) wird die Kreislaufwirtschaft als ein wesentlicher Schwerpunkt behandelt. In diesem Beitrag steht die Analyse der Abfallströme zwischen dem städtischen Bereich und dessen Umland im Vordergrund. Wesentlicher Fachpartner diesbezüglich ist die BN Umwelt GmbH. In Zusammenarbeit mit der Professur für Geodäsie und Geoinformatik der Universität Rostock wurden im Projekt eine Umfrage auf den Wertstoffhöfen in Rostock und eine GIS-basierte Situationsanalyse der Wertstoffhöfe in Rostock und dem Umland durchgeführt. 
Abfallsammelsysteme lassen sich in Hol- und Bringsysteme unterscheiden. Der Wertstoffhof als Sammelplatz für viele unterschiedliche Abfallfraktionen gehört zum Bringsystem und leistet einen wichtigen Beitrag für eine kreislauforientierte Abfallwirtschaft. Ziel ist es, besonders im Hinblick auf die aktuelle Klimaund Ressourcenschutzdiskussion, den Verbrauch von Primärressourcen zu reduzieren (Ellen MacArthur Foundation, 2015). Aus diesem Grund ist die Erhöhung der Nutzenintensität ein wichtiger Bestandteil, um die Nachhaltigkeit der Stoffkreisläufe zu verbessern (Lehmacher, 2016).

Wesentlich für die Verbesserung der Entsorgungssituation ist es, Schwachpunkte im Bringsystem zu identifizieren und Verbesserungspotenziale abzuleiten. Dabei ist es wichtig, die Nutzer der Wertstoffhöfe in die Entscheidungsprozesse einzubeziehen, da sich nur so entsprechende Bedarfe ermitteln lassen (Bilitewski et al., 2000; Urban und Halm, 2015). In jüngster Zeit wird der Ausbau des Wertstoffhofes zu einer Serviceeinrichtung diskutiert, die deutlich mehr bietet als nur die Abgabe von Abfällen (Urban und Halm, 2015). Hier spielt vor allem der „Re-Use“-Gedanke durch die Mitnahme von Altgeräten oder die Einrichtung einer Bodenbörse eine wesentliche Rolle. Ob dafür Bedarfe vorhanden sind, soll im Rahmen dieser Arbeit ermittelt werden.

Weitere Untersuchungsschwerpunkte betreffen die allgemeine Zufriedenheit, insbesondere hinsichtlich der Abgabesituation, der Erreichbarkeit und der Öffnungszeiten. Eng damit verbunden sind auch die Bedürfnisse hinsichtlich der Abfallarten. Gerade hinsichtlich der Emission von Lärm oder beispielsweise der potenziellen Belastung von Böden oder Grundwasser bei bestimmten Abfällen müssen die Höfe besondere Anforderungen erfüllen, damit diese überhaupt angenommen werden dürfen (Bilitewski et al., 2000).

Eine große Herausforderung, vor denen die Höfe stehen, ist die fortschreitende Digitalisierung. Sie ist zum einen in der Lage, dem Kunden mehr Komfort bei der Verwendung der Höfe zu bieten, aber auch der Planung durch die Datenerfassung eine deutlich bessere Datengrundlage zu liefern (Wilts und Berg, 2017). Dies wiederum unterstützt die Bevölkerung bei der Verwendung der Höfe und trägt damit der im Kreislaufwirtschaftsgesetz verankerten Hierarchie „Vermeidung - Vorbereitung zur Wiederverwendung - Recycling - sonstige Verwertung - Beseitigung" Rechnung. 


\section{$2 \quad$ Methodik}

Kern der vorliegenden Arbeit ist die Erstellung einer Umfrage, die auf den Wertstoffhöfen der Hanse- und Universitätsstadt Rostock durchgeführt wurde. Der verwendete Fragebogen wurde mithilfe der Umfragesoftware EvaSys ${ }^{1}$ erstellt. Die Umfrage wurde sowohl als Interview vor Ort als auch in Form einer Online-Umfrage durchgeführt. Für Letzteres wurden entsprechende Barcodes erstellt und auf den Wertstoffhöfen verteilt. Durch die Verwendung von EvaSys konnten die Fragebögen automatisch ausgewertet werden.

Die Umfrage gliedert sich in vier Bereiche: Zu Beginn werden allgemeine Informationen der Wertstoffhofnutzer abgefragt (Alter, Wohnsituation, Postleitzahl ...), gefolgt von der Abfrage der Fraktion der angelieferten Abfälle und der Bereitschaft zur Nutzung von gebrauchsfähigen Geräten. Die dritte Fragengruppe bezieht sich auf die Erreichbarkeit der Höfe. Hier ist insbesondere das Haupttransportmittel von Interesse. Die vierte und letzte Fragengruppe bezieht sich auf die Kundenzufriedenheit. Zuletzt wurde noch eine offene Frage formuliert, in der die Befragten Ideen und Wünsche für einen zukünftigen Wertstoffhof äußern konnten. Die Umfrage war für einen Zeitraum von zwei Monaten Ende 2019 vorgesehen.

Neben der Umfrage wurden im Rahmen dieser Arbeit auch die Wertstoffhöfe digitalisiert. Hierbei wurden sowohl Containervolumina für die einzelnen Abfallfraktionen als auch die Verkehrs- und Verwaltungsflächen erfasst. Durch die Analyse der Container- und Flächenausstattung lässt sich die Versorgungsleistung auf den Wertstoffhöfen besser parametrisieren. Diese Informationen sind ein wesentlicher Bestandteil, um aus entsprechenden Erreichbarkeitsanalysen konkrete Kapazitätsauslastungen für die Wertstoffhöfe abzuleiten.

Die Erfassung fand mittels der Digitalisierung vorhandener Lagepläne und auf Basis von Vor-Ort-Begehungen sowie Luftbildern statt. Diese Daten wurden auch für die Höfe des Landkreises erhoben, jedoch in deutlich geringerer Genauigkeit, da eine Vor-Ort-Begehung und Einmessung im Rahmen dieser Arbeit nicht vorgenommen wurde; die Höfe sind hier mittels digitaler Orthophotos erfasst worden.

${ }^{1}$ https://www.evasys.de/ 


\section{Ergebnisse}

Insgesamt konnten durch die Umfrage 202 vollständig auswertbare Fragebögen erhoben werden, davon wurden 36 Bögen online ausgefüllt. Allerdings gilt die Umfrage als nicht repräsentativ Dies wird vor allem an der nicht ausgewogenen Altersstruktur der Befragten deutlich. So waren 37,1\% der Befragten 45 bis 65 Jahre und 38,6 \% der Befragten $>65$ Jahre alt. Demgegenüber war die Altersgruppe von 15 bis 25 Jahre nur mit $2 \%$ vertreten.

Anhand der räumlichen Zuordnung lässt sich deutlich erkennen, dass die Einwohner i. d. R. den nächstgelegenen Hof anfahren (Abbildung 1). Zudem stammen die Anlieferungen nur zu einem geringen Teil von außerhalb der Stadtgrenzen Rostocks.

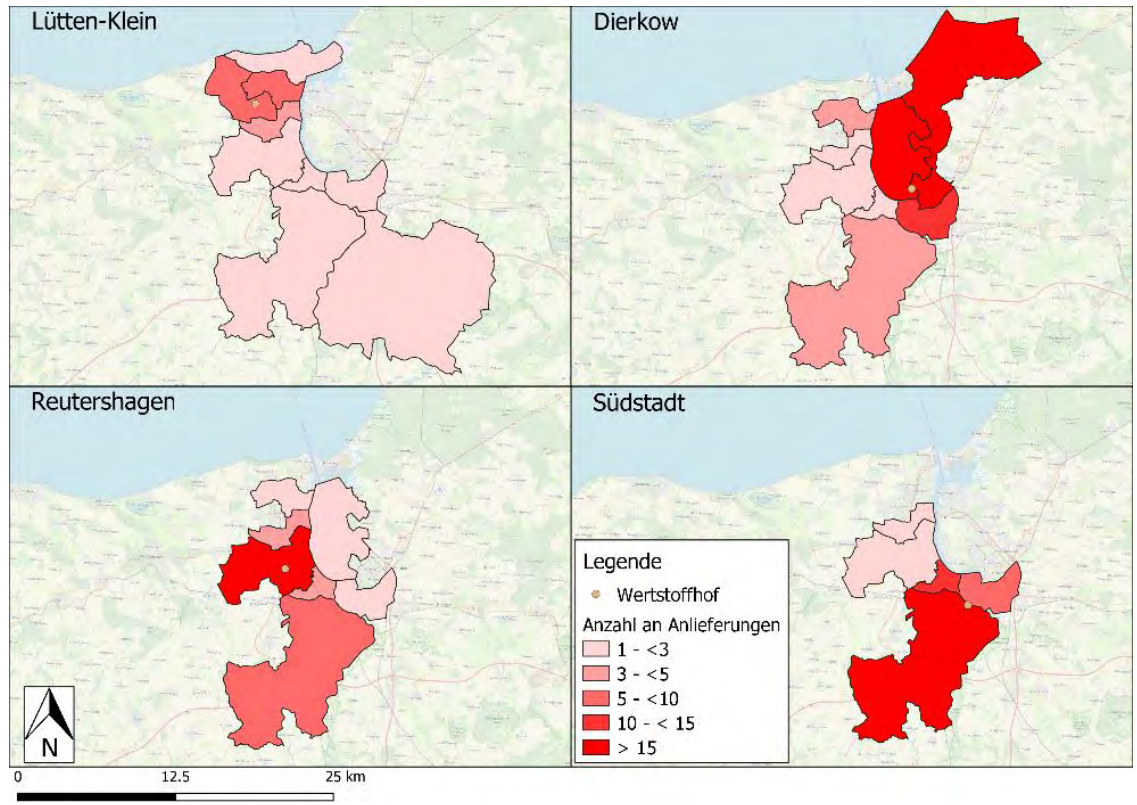

\section{Abbildung 1: Herkunft der Befragten nach PLZ (Datengrundlage: OpenStreetMap, OpenData.HRO)}

Trotz der altersbedingten Verzerrung lassen sich einige wesentliche Rückschlüsse aus der Befragung ziehen. Im Allgemeinen sind die Rostocker mit dem Ist-Zustand der Wertstoffhöfe zufrieden. Dies spiegelt sich sowohl in der Bewertung als auch in den als wichtig angesehenen Faktoren wider (Abbildung 2). 
$68 \%$ der Befragten haben keine Verbesserungswünsche genannt. Zudem zeigte die Umfrage, dass das wichtigste Element für die Befragten die Erreichbarkeit ist. Dieser Faktor ist für Rostock nach Vettermann et al. (2020) als „sehr gut“ zu bewerten. Die Kundenzufriedenheit wurde während der geführten Interviews zudem auch von einigen Befragten direkt zum Ausdruck gebracht. Dieser positive Eindruck zeigt sich gleichbleibend über alle Wertstoffhöfe der Hanse- und Universitätsstadt Rostock.

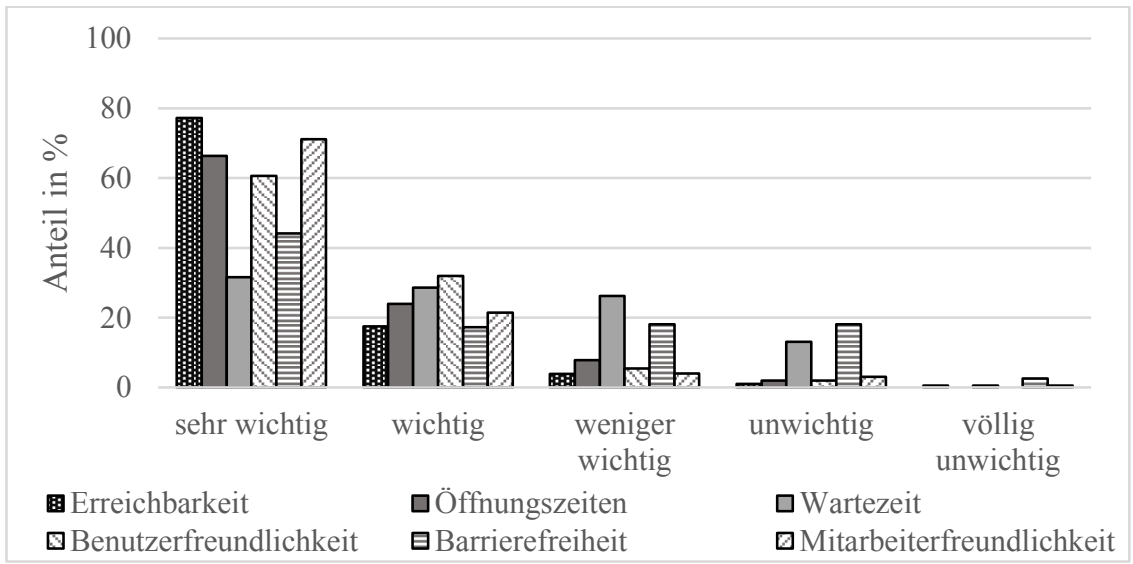

\section{Abbildung 2: Bewertung der Höfe durch die Befragten}

Eine wichtige Erkenntnis der Befragung ist die Verteilung der Abfallfraktionen der abgegebenen Abfälle. So bestand erwartungsgemäß der Großteil der Abfälle aus Grünabfällen (80\%), gefolgt von Elektroschrott (74\%), Sperrmüll (58\%) und Altfarben/Lacke (36\%). Auffallend für die Analyse der Höfe ist hierbei vor allem die Kombination der Abfallfraktionen mit der Wohnsituation (Abbildung 3). Daraus lassen sich vor allem für die Bestimmung der potenziellen Abfalldichte Rückschlüsse zur Parametrisierung ziehen. Diese wird aus der tatsächlichen Nutzung, welche aus ALKIS extrahiert wurde, bestimmt und soll einen wesentlichen Bestandteil bei der Planung neuer Höfe darstellen. Es ist ersichtlich, dass die für das Bringsystem abfallreichsten Siedlungsareale Einfamilienhäuser mit Gärten sind. Dies ist damit zu begründen, dass diese Quelle für alle relevanten Abfallfraktionen darstellen. Einwohner von Mehrfamilienhäusern liefern hingegen deutlich weniger Grünschnitt an, dessen Ursprung in den Kleingartenanlagen zu suchen ist. Die absolute Abfallmenge pro Fläche ist zudem auch hier hoch, im Wesentlichen aber bedingt durch die deutlich höhere Bevölkerungsdichte. Hinsichtlich der Anlieferungen auf den Wertstoffhöfen bestätigt sich dieser Eindruck. Verdeutlicht wird die Bedeutung der Wohnsitua- 
tion zudem durch die Abfallverteilung im Jahr 2018. Im nahen Einzugsbereich des Wertstoffhofes Südstadt ist die Fläche an Kleingärten und offenen Wohnbauflächen deutlich geringer. Der Grünschnittanteil beträgt dementsprechend nur $52 \%$, während er im Mittel über alle Wertstoffhöfe Rostocks bei $61 \%$ liegt.

Ein überraschendes Ergebnis der Befragung ist die Entsorgung von funktionsfähigen Geräten durch $71 \%$ der Befragten. Parallel sind $68 \%$ der Befragten bereit, diese weiter nutzen zu wollen. Dies ist gerade dahingehend interessant, dass so dem Kreislaufansatz direkt Rechnung getragen werden könnte. Beim Umbau der Höfe zu Servicezentren sollten somit entsprechende Flächen vorgehalten werden, um hier im Bedarfsfall einen solchen Service anbieten zu können.

Allerdings gelten diese Aspekte nur für die Stadt Rostock. In weitergehenden Untersuchungen könnte im Hinblick auf die Analyse der Stadt-LandInteraktionen der Untersuchungsraum auf das Umland ausgeweitet werden. Aufgrund der altersbedingten Verzerrung in der Befragung sollten ähnliche Erhebungen mit einer ausgewogenen Verteilung der Altersstruktur durchgeführt werden, um die Ergebnisse entsprechend verifizieren zu können.

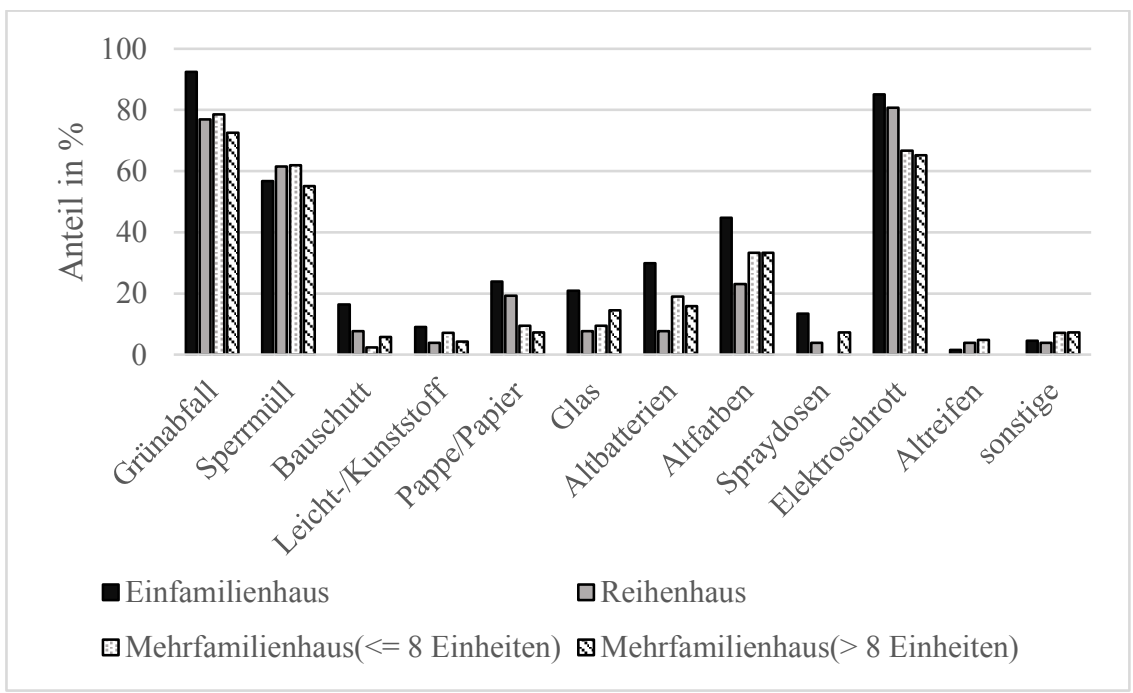

Abbildung 3: Abfallverteilung je nach Wohnsituation der Befragten 


\section{$4 \quad$ Zusammenfassung und Ausblick}

Durch die Umfrage auf den Wertstoffhöfen der Hanse- und Universitätsstadt Rostock ist es möglich, ein Stimmungsbild der Nutzer zu erstellen. Obgleich die Umfrage mit insgesamt 202 Antworten als nicht repräsentativ zu werten ist, lässt sich vermuten, dass die Rostocker durchaus zufrieden mit den vier Wertstoffhöfen im Stadtgebiet sind. Durch die Umfrage ist es zudem möglich, Rückschlüsse auf das Entsorgungsverhalten der Rostocker Bevölkerung zu schließen. Besonders für die Bestimmung der Schwerpunkte der Abfallentstehung im Rahmen des Projektes Prosper-Ro sind diese Informationen von großer Bedeutung. Durch die Umfrage wird noch einmal deutlich, dass vor allem Grünschnitt einen wesentlichen Anteil des Abfalls einnimmt. Insbesondere in Kombination mit der Wohnsituation der Befragten zeigt sich ein deutliches Bild, dass vor allem Einfamilienhaussiedlungen den größten Abfallanteil auf den Höfen verursachen. Daneben zeigt die Umfrage auch, dass der ReUse-Gedanke zukünftig Potenzial hinsichtlich der Abgabe und Mitnahme von Elektroaltgeräten bietet.

Zukünftig ist eine Erweiterung des Umfragegebietes auf den Landkreis denkbar. Dadurch wird es möglich, entsprechende Vergleiche zwischen Stadt- und Landkreis zu ziehen sowie die unterschiedlichen Gegebenheiten, Bedürfnisse und Zufriedenheiten hinsichtlich der Nutzer herauszuarbeiten. Zudem ist eine erneute Durchführung über einen längeren Zeitraum in der Stadt selbst denkbar.

\section{Literatur}

kvwmap-wiki: Was ist kvwmap? Online unter: https://kvwmap.de/index.php/Was_ist_kvwmap\%3F (Letzter Zugriff am 10.02.2019). 2017. 

GIS-Technologie II 



\title{
Open Location Code API (OLCA)
}

\author{
Web-API zur Nutzung der „Plus codes“6 \\ des Open Location Codes
}

\author{
Sebastian Gutzeit \\ Hanse- und Universitätsstadt Rostock, \\ Kataster-, Vermessungs- und Liegenschaftsamt \\ sebastian.gutzeit@rostock.de
}

\begin{abstract}
Zur global eindeutigen Beschreibung von Positionen sind die etablierten Verfahren häufig entweder zu anfällig für Notations- und Übertragungsfehler (im Falle geographischer Koordinaten), räumlich zu beschränkt (bei kartesischen Systemen), abseits bewohnter Gebiete nicht tauglich (im Falle von Adressen) oder aber staatenübergreifend nicht nutzbar (Flurstücke). Daher wurden universelle Geocodierungssysteme geschaffen, die Koordinaten in „Location codes“ umsetzen. Der Open Location Code (,Plus codes“) ragt hier mit seinen Eigenschaften als System besonders hervor, weshalb eine freie Web-API - Open Location Code API (OLCA) - geschaffen wurde, die die „Plus codes“ erschließt und für viele Einsatzbereiche nutzbar macht, etwa für Notfalldienste.
\end{abstract}

\section{$1 \quad$ Einleitung}

Um jede beliebige Position auf der Erdoberfläche eindeutig beschreiben zu können, werden in der Regel geographische Koordinaten verwendet, bestehend aus einer Längen- und einer Breitenangabe (Ogrissek, 1983). Dabei handelt es sich um ein Verfahren, das sich auch einer breiten Öffentlichkeit jenseits der Geoinformationsverarbeitung erschließt und daher bereits im Unterricht an Regelschulen gelehrt und genutzt wird (als Beispiel für die Anwendung in Schulatlanten: Diercke Weltatlas, 1992).

Jedoch bringt die Verwendung geographischer Koordinaten auch diverse Unsicherheiten mit sich: Welches Format wird für die Notation verwendet - das Sexagesimalformat mit seinen Grad-, Minuten- und Sekundenangaben oder das Dezimalformat? Welche Trennzeichen werden zwischen den einzelnen Koordi- 
natenwerten verwendet, welche zwischen mehreren Koordinaten? In welcher Reihenfolge werden die Koordinatenwerte notiert? Wie wird zwischen Breitengraden der Nord- und der Südhalbkugel unterschieden, wie zwischen Westen und Osten bei den Längengraden? Diese Liste ließe sich nahezu beliebig fortsetzen. Dazu kommt das Problem, dass bei der mündlichen oder schriftlichen Übermittlung insbesondere langer Koordinatenwerte (etwa durch die Verwendung vieler Nachkommastellen im Dezimalformat) leicht Fehler auftreten können, z. B. durch vergessene Ziffern oder „Ziffernsturz“.

Auf den ersten Blick bieten sich zur Beschreibung von Positionen auf der Erdoberfläche, das heißt für die Geocodierung (Bill \& Zehner, 2001), diverse Alternativen an, zum Beispiel kartesische Koordinatensysteme, (postalische) Adressen oder Flurstücke. Diese weisen jedoch ebenfalls eine Reihe von Besonderheiten und Einschränkungen auf, die ihre Eignung vor allem zur global eindeutigen Definition von Positionen in Frage stellen. Kartesische Koordinatensysteme etwa sind in der Regel nur für einen bestimmten Teil der Erdoberfläche nutzbar - auch das sehr gebräuchliche UTM-System lässt sich zum Beispiel nur von $84^{\circ}$ Nord bis $80^{\circ}$ Süd anwenden (Hake et al., 2002). Adressen und Flurstücke sind bei Weitem nicht in jedem Staat der Erde gebräuchlich und selbst dort, wo entsprechende Systeme existieren, sind sie zumeist stark auf die jeweiligen nationalen Gegebenheiten angepasst. Flurstücke können zudem eine sehr große Fläche aufweisen - eine Tatsache, die eine genaue Positionsangabe rein durch die Angabe von Flurstücksnummern nahezu unmöglich macht - und Adressen sind in der Regel nicht eindeutig in Bezug auf ihre Lage: Ist der Eingang eines Gebäudes maßgeblich oder dessen Mittelpunkt? Außerdem liegen viele Gebiete weit von (Gebäude- resp. postalischen) Adressen entfernt, zum Beispiel unbesiedelte Waldgebiete oder Seen.

\section{Universelle Geocodierungssysteme}

Um den aufgezeigten Problemen bei der Verwendung geographischer oder kartesischer Koordinaten sowie von Adressen oder Flurstücksnummern entgegenzutreten, wurden in der Vergangenheit mehrere universelle, also global nutzbare, Geocodierungssysteme geschaffen, mit deren Hilfe sich Punkte resp. auch kleine Flächen auf der Erde eindeutig identifizieren lassen, und zwar unabhängig von deren tatsächlichen geographischen oder kartesischen Koordinaten. Allen diesen Systemen ist gemein, dass sie auf Basis mathematischer Methoden und entsprechender Algorithmen geographische Koordinaten in sogenannte „Location codes“ konvertieren (Google LLC, 2018). 
In der nachfolgenden Tabelle werden die relevantesten (Google LLC, 2018) Systeme im Hinblick auf ihre freie Nutzbarkeit gegenübergestellt. Weiterhin wird verglichen, ob sie in mehreren Raster- und damit Genauigkeitsstufen (resultierend in kürzeren/längeren Codes resp. kleineren/größeren Flächen, die repräsentiert werden) verwendet werden können und ob benachbarte Koordinaten in ähnliche „Location codes“ konvertiert werden (und somit auf den ersten Blick als benachbart erkennbar sind). Außerdem wird in der Tabelle die tatsächliche Code-Syntax aufgezeigt, und zwar am Beispiel der Position 54,09295 Nord und $12,09801^{\circ}$ Ost (es handelt sich dabei um den Haupteingang des Hauses des Bauens und der Umwelt, Holbeinplatz 14, Rostock):

Tabelle 1: Vergleich der relevantesten (Google LLC, 2018) universellen Geocodierungssysteme

\begin{tabular}{|c|c|c|c|c|}
\hline System & frei? & $\begin{array}{l}\text { Raster- } \\
\text { stufen }\end{array}$ & $\begin{array}{c}\text { Nachbar- } \\
\text { koordinaten }= \\
\text { ähnliche Codes? }\end{array}$ & $\begin{array}{c}\text { „Location Code“ für Holbeinplatz } \\
\text { 14, } \\
\text { Rostock }\end{array}$ \\
\hline $\begin{array}{c}\text { 3GeoNames } \\
{[1]}\end{array}$ & ja & keine & ja & NAKSKOV-CARDIN-BESITZEN \\
\hline $\begin{array}{c}\text { Geohash } \\
{[2]}\end{array}$ & ja & 6 & ja & u38eer3d20d \\
\hline $\begin{array}{c}\text { Maidenhead } \\
\text { Locator System } \\
\text { [3] }\end{array}$ & ja & 6 & ja & JO64bc12 \\
\hline $\begin{array}{c}\text { Makaney Code } \\
{[4]}\end{array}$ & ja & keine & ja & $\mathrm{XBRH}+2 \mathrm{M} 22$ \\
\hline $\begin{array}{c}\text { Mapcode } \\
{[5]}\end{array}$ & ja & keine & ja & WJJR9.TD53 \\
\hline $\begin{array}{c}\text { Natural Area } \\
\text { Code [6] }\end{array}$ & nein & keine & nein & J07C3 S0FX6 \\
\hline $\begin{array}{l}\text { Open Location } \\
\text { Code } \\
{[7]}\end{array}$ & ja & $5(6)$ & ja & $9 \mathrm{~F} 6 \mathrm{~J} 33 \mathrm{VX}+56$ \\
\hline $\begin{array}{c}\text { Open Postcode } \\
{[8]}\end{array}$ & ja & 8 & ja & 4WTWN9VQC \\
\hline $\begin{array}{c}\text { what3words } \\
\text { [9] }\end{array}$ & nein & keine & nein & ///zutreffen.ausführen.bürste \\
\hline
\end{tabular}

[1: Ruci, 2019; 2: Wikipedia, 2020a; 3: Wikipedia, 2020b; 4: Al-Salloum, 2011; 5: Wikipedia, 2020c; 6: NAC Geographic Products Inc., 2020; 7: Rinckes \& Bunge, 2019; 8: Wikipedia, 2020e; 9: Wikipedia, 2020f] 
Im Jahr 2019 hatte das Kataster-, Vermessungs- und Liegenschaftsamt der Hanse- und Universitätsstadt Rostock (KVLA-HRO) die Aufgabe, ein passendes, global nutzbares Geocodierungssystem zu identifizieren, das nachfolgende Anforderungen erfüllt: freie Nutzbarkeit, Abdeckung mehrerer Raster- und damit Genauigkeitsstufen (ca. $1 \mathrm{~km} \times 1 \mathrm{~km}$, ca. $100 \mathrm{~m}$ x $100 \mathrm{~m}$ und ca. $10 \mathrm{~m} \times 10 \mathrm{~m}$ ), ähnliche „Location codes“ für benachbarte Koordinaten, keine Wörter oder zufälligen Wortbildungen sowie keine leicht verwechselbaren Zeichen in der Syntax der „Location codes“. Diese Anforderungen entstammen den fachlichen Bedürfnissen der städtischen Feuerwehr, die diese Aufgabe maßgeblich initiiert hat. Anhand des bereits vorgestellten, tabellarischen Vergleichs sowie als Resultat tiefergehender Prüfungen der einzelnen Systeme fiel die Wahl auf den Open Location Code, der 2014 im Auftrag des Softwareunternehmens Google LLC entwickelt wurde. Die Systeme, die zumindest laut Tabelle ebenfalls die genannten Anforderungen erfüllen, weisen leicht verwechselbare Zeichen und teilweise auch Wortbildungen in der Syntax auf (Geohash, Maidenhead Locator System und Open Postcode); beim Open Postcode kommt es zudem häufig vor, dass unmittelbar benachbarte Koordinaten resp. Flächen stark unterschiedliche „Location codes" aufweisen und somit nicht auf den ersten Blick als benachbart erkannt werden. Ein weiterer Grund, der für die Wahl des Open Location Codes als weiter zu betrachtendes Geocodierungssystem spricht, ist dessen Nutzbarkeit im „Google-Kosmos“, das heißt vor allem in den bekannten Anwendungen Google Maps und Google Earth, die unter anderem von der Feuerwehr in Ergänzung des städtischen Angebotes an Geodatendiensten und -portalen genutzt werden.

\section{Open Location Code und die „Plus codes“}

Das Geocodierungssystem Open Location Code zeichnet sich insbesondere dadurch aus, dass alle resultierenden „Location codes“ - die sogenannten „Plus

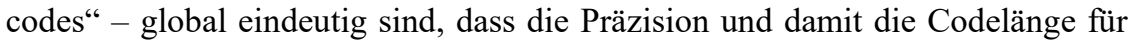
Raster- (fünf) und Genauigkeitsstufen (sechs) variabel ist, dass ihm eine logische, rein mathematische Definition zugrunde liegt, dass die Codes keine leicht verwechselbaren Zeichen - wie etwa 1 vs. I oder 0 vs. $\mathrm{O}$ - enthalten, dass es nicht zu (versehentlichen) Wortbildungen kommen kann, und dass es vollständig Open source und damit frei nutzbar ist (Rinckes \& Bunge, 2019).

Der Aufbau der „Plus codes“ basiert auf dem Netzgitter der geographischen Längen- und Breitengrade: 
- 2-stelliger Code (Raster-/Genauigkeitsstufe resp. Level 1) entspricht einem Quadrat mit Kantenlänge $20^{\circ}$ (in MecklenburgVorpommern (MV) ca. $2.200 \mathrm{~km}$ x $1.300 \mathrm{~km}$ ); Beispiel: 9F000000+ (in langschriftlicher Notation) resp. 9F (in Kurzform)

- $\quad$ 4-stelliger Code (Level 2) $\triangleq$ Quadrat mit $1^{\circ}$ (MV ca. $111 \mathrm{~km} \mathrm{x} 65$ $\mathrm{km}$ ); Beispiel: 9F6J0000+ resp. 9F6J

- $\quad$ 6-stelliger Code (Level 3) ^ Quadrat mit $0,05^{\circ}$ (MV ca. $5.600 \mathrm{~m}$ x $3.300 \mathrm{~m}$ ); Beispiel: 9F6J3300+ resp. 9F6J33

- $\quad 8$-stelliger Code (Level 4) $\triangleq$ Quadrat mit $0,0025^{\circ}$ (MV ca. $278 \mathrm{~m}$ x $164 \mathrm{~m}$ ); Beispiel: 9F6J33VX+

- $\quad$ 10-stelliger Code (Level 5) $\triangleq$ Quadrat mit $0,000125^{\circ}$ (MV ca. 14 m x 8 m); Beispiel: 9F6J33VX+54

- wenig gebräuchlicher 11-stelliger Code (Genauigkeitsstufe resp. Level 6) entspricht einem Rechteck mit Kantenlängen 0,000025 und $0,00003125^{\circ}$ (MV ca. $3 \mathrm{~m} \times 2 \mathrm{~m}$ ); Beispiel: 9F6J33VX+54P
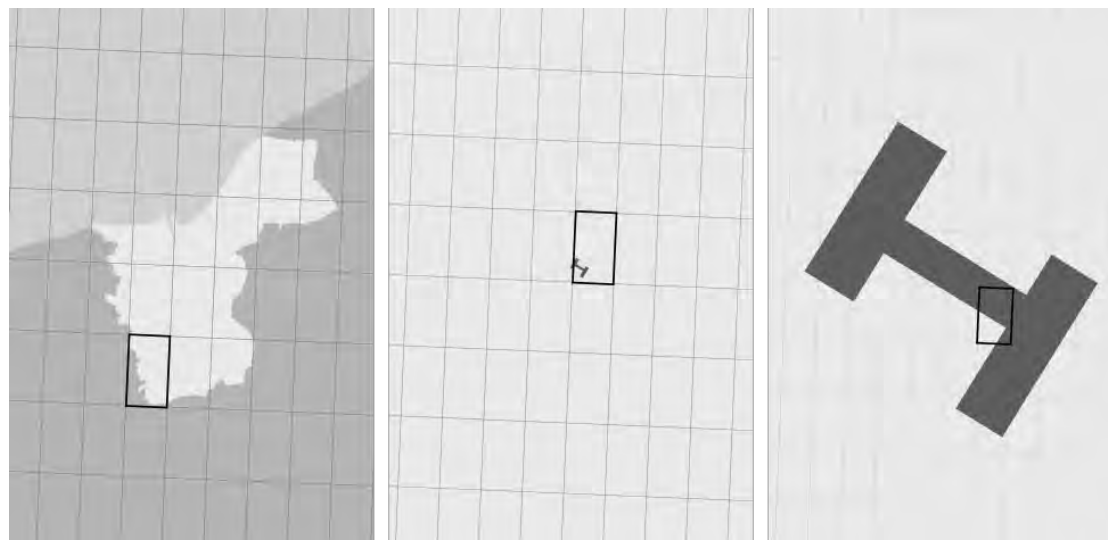

Abbildung 1: links - Level 3, 6-stelliger Code, 9F6J3300+; Mitte - Level 4, 8stelliger Code, 9F6J33VX+; rechts - Level 5, 10-stelliger Code, 9F6J33VX+54

„Plus codes“ werden offiziell mindestens 8-stellig notiert, gefolgt von einem „+" (daher die Bezeichnung „Plus codes“) - Beispiele: 9F000000+ oder 9F6J0000+. Auf den Levels 1, 2 und 3 (2-, 4- und 6-stellige Codes) werden die „auffüllenden“ Nullen in der Regel fortgelassen - Beispiele: 9F oder 9F6J. Alle weiteren Stellen (auf Level 5 zwei weitere Stellen, auf Level 6 drei) werden nach dem ,+“ notiert - Beispiele: 9F6J33VX+54 oder 9F6J33VX+54P. Die Syntax lässt die folgenden 20 Zeichen zu: 2, 3, 4, 5, 6, 7, 8, 9, C, F, G, H, J, M, 
P, Q, R, V, W und X. Aus dem „quadratischen“ Aufbau folgt demnach, dass je Rasterstufe exakt 20 x 20, also 400, Zeichenpaare zulässig sind - eine Erhöhung des Levels führt bis einschließlich Level 5 zu zwei Stellen mehr im Code, auf Level 6 dann zu drei Stellen mehr (Rinckes \& Bunge, 2019).

Bei Verwendung eines Referenzstandortes können die ersten vier Stellen eines „Plus codes“ mit mindestens acht Stellen fortgelassen werden, wenn sowohl der Breitengrad als auch der Längengrad des Referenzstandortes innerhalb von +/$0,25^{\circ}$ des Breitengrads (also +/- ca. $28 \mathrm{~km}$ in MV) und Längengrads (also +/- 16 $\mathrm{km}$ in MV) des Zentrums des Codes liegen. Stattdessen wird dann die Bezeichnung des Referenzstandortes verwendet, sodass sich ein sogenannter „Regionalcode“ ergibt - Beispiel: aus 9F6J33VX+54 wird 33VX+54 Rostock oder Rostock 33VX+54.

\section{Open Location Code API (OLCA)}

Zur Etablierung des Open Location Codes auch jenseits des „Google-Kosmos“ und zwecks Möglichkeit der Einbindung in die Geodateninfrastruktur der Stadtverwaltung hat das KVLA-HRO die Open Location Code API (OLCA) geschaffen - eine Web-Programmierschnittstelle (application programming interface, API) zur Konvertierung von Koordinaten in „Plus codes“ des Open Location Codes und umgekehrt. Sie kann zum Beispiel in Online-Portale eingebunden werden, um diese mit einer entsprechenden Suche oder Konvertierungsfunktion auszustatten. In einem speziellen „Kartenmodus“ dient OLCA zudem auch der massenhaften Erzeugung von Codes innerhalb definierter Gebiete: Somit lässt sich beispielsweise die Datengrundlage für Web-Dienste dynamisch erzeugen, etwa für einen Web Map Service (WMS).

OLCA ist vollständig in Python entwickelt. Als Basis, das heißt in erster Linie für die Konvertierungsfunktion, wird die entsprechende Open-Location-CodeSoftwarebibliothek verwendet, die unter einer Freien-Software-Lizenz beim Onlinedienst GitHub veröffentlicht ist: https://github.com/google/open-locationcode. Die API ist direkt auf Servern der Hanse- und Universitätsstadt Rostock nutzbar und kann unter den API-Endpunkten https://geo.sv.rostock.de/olca/ (für die Such-/Konvertierungsfunktionen) sowie https://geo.sv.rostock.de/olca/map (für den „Kartenmodus“) angesprochen werden; auf der statischen Webseite https://geo.sv.rostock.de/olca.html findet sich außerdem eine Kurzübersicht.

Unterstützt werden die HTTP-Methoden GET und POST; Letztere kann entweder mittels Formulardaten (also Content-Type: application/x-www- 
form-urlencoded) oder mittels Body in JavaScript Object Notation (JSON) (also Content-Type: application/json) genutzt werden. Beispiele:

- GET-Aufruf - Suche nach einem Koordinatenpaar: https://geo.sv.rostock.de/olca/?query=12.097875,54.092875

- GET-Aufruf - Suche nach einem „Regionalcode“: https://geo.sv.rostock.de/olca/?query $=33 \mathrm{VX}+55$,rostock

- GET-Aufruf - Erzeugung aller „Plus codes“ innerhalb einer „Bounding-Box“" via „Kartenmodus“: https://geo.sv.rostock.de/olca/map?bbox=12.51,54.22,12.513,54.222

Die API liefert alle erfolgreichen Resultate in validem GeoJSON zurück, das als Geometrie (geometry) die Fläche des gefundenen „Plus codes“ als Polygon umfasst und als Attribut (unter properties) unter anderem die vollständige Notation des Codes. Nicht erfolgreiche Resultate (Fehler) werden in JSON mit entsprechender Meldung und einem passenden HTTP-Statuscode ausgegeben.

Sowohl im Standard- als auch im „Kartenmodus“ lässt sich die API mit optionalen Zusatzparametern aufrufen: Beispielsweise lässt sich über epsg_out das Koordinatenreferenzsystem bestimmen, in dem alle zurückgelieferten Koordinaten vorliegen sollen.

Für die Geocodierung von Referenzstandorten im Zusammenhang mit den oben beschriebenen „Regionalcodes“ nutzt OLCA serverseitig Nominatim, einen frei nutzbaren Geocodierer, der auf den Daten des freien Kartendatenprojektes OpenStreetMap (OSM) aufsetzt.

OLCA ist eine Open-Source-Software und unter der Freien-Software-Lizenz Apache License 2.0 auf GitHub veröffentlicht: https://github.com/rostock/olca. Somit ist die Software ohne Einschränkungen frei nutzbar. Alle Informationen bezüglich der Installation, Konfiguration sowie der Arbeit mit OLCA (inklusive API-Dokumentation) können in den entsprechenden Erläuterungen unter https://github.com/rostock/olca/blob/master/README.md nachgelesen werden.

\section{$5 \quad$ Einsatz der OLCA}

Die möglichen Einsatzgebiete für den Open Location Code und damit auch für die API erstrecken sich über alle Bereiche, die auf Geoinformationen und dabei vor allem auf genaue sowie eindeutige Verortungen angewiesen sind - auch und gerade in Gebieten abseits von Gebäuden und (postalischen) Adressen. In erster 
Linie sind hier natürlich Notfall- und Einsatzdienste sowie die Katastrophenhilfe zu nennen: Hier läuft die Positionsbestimmung vielfach über (Sprech-)Funk, sodass Übermittlungsfehler weitreichende Folgen haben können.

Aktuell ist OLCA bereits in Geoport.HRO eingebunden, dem Portal für Geodaten der Hanse- und Universitätsstadt Rostock und Umgebung: Wenn man dort die Suche nach Open Location Codes („Plus codes“) startet, nutzt man nichts anderes als OLCA. Auch in der Anschau-/Druckanwendung der Offenen Regionalkarte Mecklenburg-Vorpommern (ORKa.MV) ist OLCA eingebunden. Angeboten wird zudem ein WMS mit den weltweiten „Plus codes“, der zu Visualisierungszwecken ebenfalls in Geoport.HRO eingebunden ist. Im Open-DataPortal der Hanse- und Universitätsstadt Rostock schließlich steht ein Teil der „Plus codes“ des Open Location Codes zum Download bereit.

\section{$6 \quad$ Fazit und Ausblick}

Der Open Location Code soll sich mittels OLCA langfristig innerhalb der Geodateninfrastruktur der Stadtverwaltung Rostock etablieren und somit zu einer auch in der Breite häufig genutzten Variante der Positionsbestimmung werden gerade dort, wo Adressen, Flurstücke usw. nicht zielführend sind.

Da nun auch die Öffentlichkeit über OLCA die „Plus codes“ bequem nutzen kann - und dies vor allem außerhalb des „Google-Kosmos"“ -, sollte dies zu (Einsatz-)Möglichkeiten führen, die hier und jetzt noch nicht absehbar sind, die aber sicherlich vielfältiger und näher am Alltag sind als die zuvor aufgezeigten Notfall- und Einsatzdienste oder die Katastrophenhilfe. Ein Beispiel könnte die Fahrzeugnavigation sein: Ziele ohne Adressen in unbekannten Umgebungen, etwa im Rahmen von Urlaubsreisen, könnten mithilfe der „Plus codes“ sicherlich direkter und treffsicherer angesteuert werden als mittels fehleranfälliger (und oft umständlicher) Koordinateneingabe oder anderer Methoden der Zieleingabe.

Im Übrigen sind alle Variablennamen und Kommentare im Quellcode der Open Location Code API sowie deren Dokumentation in englischer Sprache verfasst, sodass auch eine internationale Öffentlichkeit OLCA nutzen und damit unter Umständen Einsatzgebiete in Ländern erschließen kann, die beispielsweise ohne postalische Adressen auskommen: Hier könnten „Plus codes“ womöglich das Post-/Zustellwesen unterstützen. Tatsächlich ist dies in Kap Verde bereits heute Praxis (Wikipedia, 2020d). 


\section{Literatur}

Al-Salloum, Z. S. (2011): Makaney Code. In: Makaney Code. 2011. URL: http://www.makaney.net/mkc_standard.html (27. Februar 2020).

Bill, R., Zehner, M. (2001): Lexikon der Geoinformatik. Wichmann.

Diercke Weltatlas (1992): 3. Aufl., Westermann.

Google LLC (2018): An Evaluation of Location Encoding Systems. In: GitHub. 10. Dezember 2018. URL: https:/github.com/google/open-locationcode/wiki/Evaluation-of-Location-Encoding-Systems (27. Februar 2020).

Hake, G., Grünreich, D., Meng, L. (2002): Kartographie - Visualisierung raum-zeitlicher Informationen. 8. Aufl., de Gruyter.

NAC Geographic Products Inc. (2020): The Natural Area Coding System. In: The Natural Area Coding System. 2020. URL: http://www.nacgeo.com/nacsite/documents/nac.asp (27. Februar 2020).

Ogrissek, R. (1983): ABC Kartenkunde. Brockhaus.

Rinckes, D., Bunge, P. (2019): Open Location Code - An Open Source Standard for Addresses, Independent of Building Numbers And Street Names. In: GitHub. 21. April 2019. URL: https://github.com/google/open-locationcode/blob/master/docs/olc_definition.adoc (27. Februar 2020).

Ruci, E. (2019): 3GeoNmes.org - An open source Geocoding system for the simple communication of locations with a resolution of $1 \mathrm{~m}$. In: FOSDEM. 3. Februar 2019. URL: https://archive.fosdem.org/2019/schedule/event/geo_3geonames/ (27. Februar 2020).

Wikipedia (2020a): Geohash. In: Wikipedia - Die freie Enzyklopädie. 22. Februar 2020. URL: https://en.wikipedia.org/wiki/Geohash (27. Februar 2020).

Wikipedia (2020b): Maidenhead Locator System. In: Wikipedia - Die freie Enzyklopädie. $20.2020 . \quad$ Januar https://en.wikipedia.org/wiki/Maidenhead_Locator_System (27. Februar 2020).

Wikipedia (2020c): Mapcode. In: Wikipedia - Die freie Enzyklopädie. 5. Januar 2020. URL: https://en.wikipedia.org/wiki/Mapcode (27. Februar 2020).

Wikipedia (2020d): Open Location Code. In: Wikipedia - Die freie Enzyklopädie. 17. Januar 2020. URL: https://en.wikipedia.org/wiki/Open_Location_Code (28. Februar 2020).

Wikipedia (2020e): OpenPostcode. In: Wikipedia - Die freie Enzyklopädie. 17. Februar 2020.

URL: https://en.wikipedia.org/wiki/Postal_addresses_in_the_Republic_of_Ireland\#O penPostcode (27. Februar 2020).

Wikipedia (2020f): what3words. In: Wikipedia - Die freie Enzyklopädie. 16. Februar 2020. URL: https://en.wikipedia.org/wiki/What3words (27. Februar 2020). 



\title{
Geocaching als Ausdruck von Mensch-Raum- Beziehungen
}

\author{
Matthias Hinz \\ Universität Rostock, Agrar- und Umweltwissenschaftliche Fakultät, \\ Professur für Geodäsie und Geoinformatik \\ matthias.hinz@uni-rostock.de
}

\begin{abstract}
Nutzergenerierte Daten aus sozialen Plattformen des Internets beinhalten oft viele raumbezogene Informationen, die etwas über die Gesellschaft und ihre Beziehung zur Umwelt aussagen. Geocaching - als ein weltweit verbreitetes Hobby mit einem starken offline- und online-Bezug - ist dabei von besonderem Interesse. Für eine offene Lerneinheit des Projekts OpenGeoEdu wurde die Plattform OpenCaching.de ausgewertet, um Erkenntnisse über die räumliche Verteilung von GeocachingAktivitäten und deren gesellschaftlichen wie umweltbezogenen Einflussfaktoren zu gewinnen. Im Rahmen einer für fortgeschrittene GIS-Nutzer konzipierten Übung in der Lerneinheit Open Data von OpenGeoEdu können die hier vorgestellten Ergebnisse durch die Kursteilnehmer reproduziert oder in den Fragestellungen adaptiert werden.
\end{abstract}

\section{$1 \quad$ Einleitung}

Geocaching ist seit 20 Jahren ein beliebter Sport, dem besonders junge Menschen sowohl im Alltag als auch während des Urlaubs nachgehen. Die Suche nach einem versteckten Schatz, dem Geocache, stellt für viele eine Motivation dar, um Orte aufzusuchen, die sie sonst möglicherweise nie betreten hätten, oder um überhaupt Zeit mit körperlicher Bewegung in der Natur zu verbringen. Nicht selten lernen Geocacher dabei Neues über die Kultur, Geschichte oder die natürlichen Gegebenheiten der besuchten Orte (O'Hara, 2008). Interessante Geocaches können also die Ferien- und Freizeiterlebnisse an bestimmten Orten besonders aufwerten. Umgekehrt könnten Häufung und Beliebtheit von Geocaches Indikatoren für die Attraktivität eines Ortes für Besucher sein. Die Untersuchung von Geocaching als gesellschaftliches Phänomen und als Ausdruck von Mensch-Raum-Beziehungen dürfte daher für eine Vielzahl weiterer Interes- 
sengruppen spannend sein, etwa in Wissenschaft, Tourismus und in der Raumplanung.

OpenCaching.de ist die zweitgrößte Geocaching-Plattform in Mitteleuropa. Sie umfasst ein Register von über 26.000 aktiven Geocaches. Jeder Eintrag beinhaltet unter anderem Hinweise zur Lage und Schwierigkeit eines Caches - Empfehlungen, wie oft ein Cache gefunden oder wie oft er gesucht und nicht gefunden wurde. Im Gegensatz zu den kommerziellen Konkurrenzplattformen können sämtliche Metadaten strukturiert über eine offene Web-API ausgelesen werden. Damit bietet die Plattform hervorragende Möglichkeiten für geostatistische Auswertungen, die von beliebigen Personen nachvollzogen werden können. Dies wurde vom Projekt OpenGeoEdu als Best-Practice-Beispiel für Forschung und Lehre aufgegriffen, um eine Lerneinheit für den Umgang mit nutzergenerierten Daten zu kreieren.

\section{$2 \quad$ Stand der Forschung}

Goldbeck und Neustaedter (2016) haben Daten von 23.318 Geocaches in den USA und Kanada ausgewertet und auf Postleitzahl- und Zensusgebiete aggregiert. Gebiete mit mehr Caches waren durchschnittlich dünner besiedelt. Gebiete, in denen Caches öfter besucht wurden, waren stärker urban geprägt und korrelierten u. a. mit einer jüngeren, gebildeteren und einkommensstärkeren Bevölkerung sowie wärmerem und niederschlagsärmerem Wetter. Rosario et al. (2019) untersuchten ca. 50.000 Geocaches in Portugal und verglichen dabei offenbarte Präferenzen aus der Anzahl an Logs pro Cache und geäußerte Präferenzen aus Textlänge der Logs, Anzahl der Bilder, Votes und Empfehlungen pro Caches mit räumlichen Einflussfaktoren - darunter Landnutzungskategorien, Höhe des Standorts, Variabilität der Landschaft, Schwierigkeit des Geocaches und die Kulturlandschaft Montado als besonderen Standort. Weitere Auswertungen von Geocaching-Daten in Portugal erfolgten durch Santos et al. (2012), rückblickend auf die Entwicklung der landesweiten Aktivitäten, und durch Mendes et al. (2013), bezogen auf die Stadt Lissabon.

Im deutschsprachigen Raum untersuchten Cord et al. (2015) für die Stadt Leipzig offenbarte Präferenzen aus der Anzahl von Geocaching-Logs pro Jahr pro Cache u. a. in Relation zu Landnutzungsdaten, der Nähe zum Stadtzentrum und zu hochfrequentierten Bereichen (z. B. in der Nähe wichtiger Hotels und Freizeiteinrichtungen). Diese verglichen sie mit geäußerten Präferenzen aus analog strukturierten Umfragen, welche jedoch in einigen Punkten den offenbarten Präferenzen zu widersprechen schienen. Weber und Haug (2012) theoretisierten 
für Deutschland Synergien und Konfliktpotenziale von Geocaching mit Akteuren im gemeinsam genutzten Raum. Hödl und Pröbstl-Haider (2017) haben Geocaching-Daten für österreichische Nationalparks ausgewertet und dabei mögliche Auswirkungen auf Naturschutzgebiete und damit verbundene Risiken evaluiert.

\section{Datensätze und Methoden}

Die hier verwendeten Datensätze umfassen vorwiegend den europäischen Raum und sind

1. ein Datenbankabbild von OpenCaching.de (JSON-Archiv / Punktdaten), Stand Oktober 2019,

2. die NUTS-Regionen (GeoJSON-Dateien / Polygone), 2016, Level 0-3

(C) EuroGeographics) ${ }^{1}$,

3. die Tabellen demo_r_pjanaggr3 (Bevölkerung) und tour_occ_nin2d (Übernachtungen / Tourismus) als CSV-Dateien aus der EurostatDatenbank sowie

4. CORINE Land Cover-Daten vom Copernicus Projekt der EU, Auflösung 25 ha / $10 \mathrm{~m}$ als geotiff-Raster ${ }^{2}$.

Aus der Datenbank wurden nur solche Geocaches ausgewertet, die zum Abfragezeitpunkt aktiv waren und sich innerhalb des Untersuchungsgebietes, des DA-CH-LI-Raums, befinden. Außerdem wurden Caches vom Typ Event, Virtuell und Quiz ausgeschlossen, da diese in der Regel nur einen losen Bezug zu physischen Aktivitäten im realen Raum haben. Alle Datensätze erlauben eine Weiterverwendung unter Beachtung der Nutzungsbedingungen, welche jedoch nur im Falle der Geocaching-Daten als Lizenz standardisiert sind, nämlich der CC BYNC-ND 3.0 DE-Lizenz. Auf explizite Anfrage an das OpenCaching.de-Team wurde eine statistische Auswertung, vorbehaltlich der von Nutzern verfassten Texte, Bilder und Loginhalte, im Rahmen der Lerneinheit und dieser Arbeit erlaubt.

Abbildung 1 zeigt den sequentiellen Ablauf der Datenanalyse. Im Zuge der Datenaufbereitung werden drei Workflow-Modelle bereitgestellt, welche den im

\footnotetext{
${ }^{1}$ https://ec.europa.eu/eurostat

${ }^{2}$ https://land.copernicus.eu/pan-european/corine-land-cover
} 
Detail etwas komplexen Ablauf des Imports i) der Geocaching-Daten, ii) der NUTS-Regionen und iii) der CORINE Land Cover-Daten in die PostGISDatenbank automatisieren. In den ersten beiden Fällen knüpft der Datenabruf direkt an die jeweilige Web-API an. Aus den europäischen NUTS-Regionen werden anhand Länderkennungen in der Attributtabelle die Regionen des D-ACH-LI-Raums gefiltert. Diese werden dann als Maske zum Zuschneiden (Clip/Overlay) der Landnutzungsdaten (in QGIS) und der GeocachingPunktdaten (in PostGIS) verwendet.

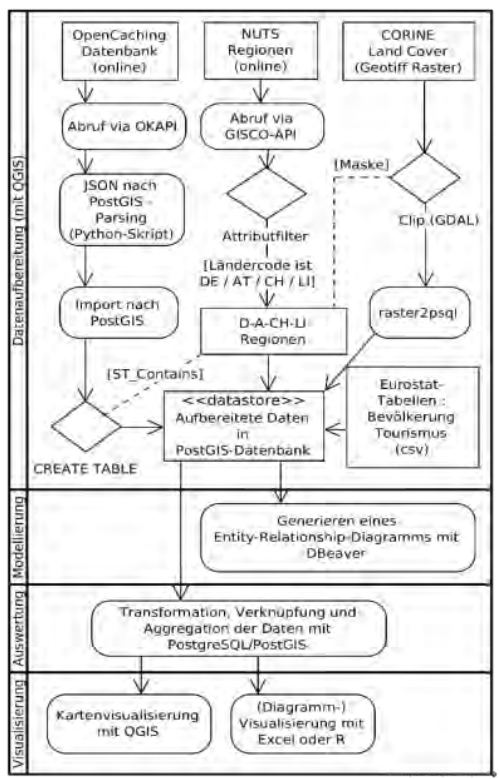

Abbildung 1: Aktivitätsdiagramm zum Ablauf der Datenanalyse, erstellt mit Visual Paradigm Community-Edition

Innerhalb der PostGIS-Datenbank erfolgen weitere Datenumformungen und die Verknüpfung und Aggregation der Datensätze mittels SQL-Anweisungen. Verschiedene Programme können direkt auf die Datenbank zugreifen, um die Daten zu visualisieren. Darunter DBeaver zum Generieren eines physischen EntityRelationship-Modells, QGIS zur Erstellung von Karten (u. a. Choroplethenkarten und Heatmaps) und wahlweise R oder Microsoft Excel zur Visualisierung von Tabellen und Diagrammen. Alle Bearbeitungsschritte können optional mit frei verfügbarer und kostenloser Software durchgeführt werden. 


\section{$4 \quad$ Ergebnisse}

Betrachtet man in Tabelle 1 die Verteilung der im Ergebnisdatensatz enthaltenen 16.939 Caches auf Länderebene, so lassen sich verschiedene Erkenntnisse gewinnen. Relativ zur Einwohnerzahl haben Deutschland und Österreich eine vergleichbare Menge an Geocaches. Jedoch werden, gemessen an der Anzahl der Logs, Geocaches in Deutschland deutlich häufiger gesucht. Für die Schweiz dagegen können nach diesem Ansatz nur wenige Geocaches ausgewertet werden. Liechtenstein weist als relativ kleines, urban geprägtes Gebiet eine hohe Dichte an Geocaches auf, wie es beispielsweise auch für die deutschen Stadtstaaten Hamburg, Bremen und Berlin der Fall ist.

Tabelle 1: Verteilung der Geocaches nach Ländern und found/notfound-Logs pro Einwohner

\begin{tabular}{|l|c|c|c|c|}
\hline \multicolumn{1}{|c|}{ Land } & Anzahl & $\begin{array}{c}\text { Anzahl } \\
\text { je 100 Tsd. EW }\end{array}$ & $\begin{array}{c}\text { Founds + notfounds } \\
\text { je 100 Tsd. EW }\end{array}$ & $\begin{array}{c}\text { Anzahl } \\
\text { je 10 } \mathbf{~ k m}\end{array}$ \\
\hline Deutschland & 15.478 & 18,7 & 295,08 & 4,33 \\
\hline Österreich & 1.325 & 15,02 & 98,15 & 1,58 \\
\hline Schweiz & 113 & 1,33 & 3,56 & 0,27 \\
\hline Liechtenstein & 30 & 78,71 & 173,17 & 18,75 \\
\hline
\end{tabular}

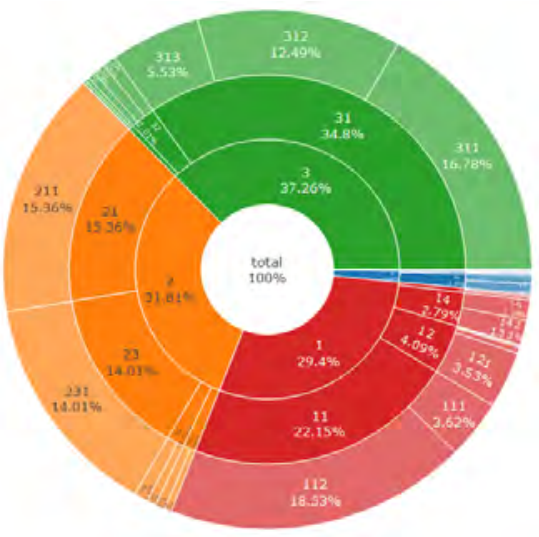

(3) Wălder und naturnahe Flächen

(2) Landwirtschaft

(1) Bebaute Flache

(5) Wasserflachen

Abbildung 2: Verteilung von Geocaches nach CORINE Land Cover-Klassen, erstellt mit Plotly für $\mathbf{R}$ 
Schlüsselt man die Verteilung der Geocaches nach Landnutzungskategorien auf (siehe Abbildung 2), so fallen unter den Naturflächen (CLC Code 3) 34,8 \% aller Caches auf Wälder (Code 31), genauer 16,78 \% Laubwälder (Code 311), 12,49 \% Nadelwälder (Code 312) und 5,53 \% Mischwälder (Code 313). Auf Busch- und Krautvegetation (Code 32) sowie offene Flächen (Code 33) entfallen zusammen nur 2,46 \% der Caches. Unter landwirtschaftlichen Flächen fallen $15,36 \%$ aller Caches auf bewässertes Ackerland (Code 211) und 14,01\% auf Wiesen- und Weideflächen (Code 231). Im Allgemeinen werden Geocaches in jeder Landnutzungskategorie etwa gleich oft besucht, das heißt, das Suchverhalten von Geocachern scheint keine bestimmten Landschaftstypen zu bevorzugen, sondern sich, wie bereits Rosario et al. (2019) feststellten, an der Verfügbarkeit der Caches zu orientieren.

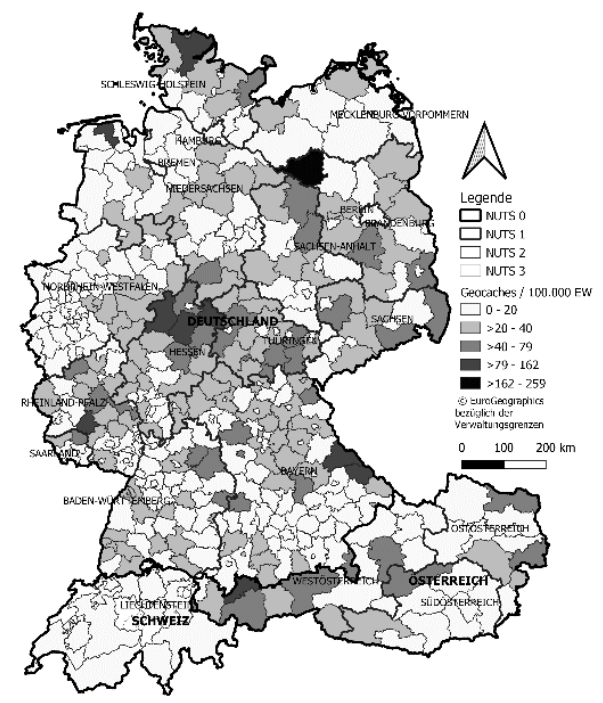

Abbildung 3: Anzahl der Geocaches pro 100.000 Einwohner nach NUTS-Regionen, klassifiziert nach natürlichen Unterbrechungen (Jenks), erstellt mit QGIS 3.10.1

Bei regionaler Betrachtung der NUTS-3-Regionen (siehe Abbildung 3) zeigt sich eine starke Ungleichverteilung der Geocaches: Besitzt ein Großteil der Regionen weniger als 41 Geocaches pro 100.000 Einwohner, so gibt es einige wenige Cluster mit einem sehr hohen Aufkommen. Geocaches pro Einwohner und Bevölkerungsdichte sind negativ korreliert (Spearman-Korrelation -0,4351, p-value <2e-16). Einige der Regionen mit dem höchsten Pro-EinwohnerAufkommen an Geocaches, wie etwa die Region Prignitz in Brandenburg oder 
die Region Außerfern in Tirol, sind gleichzeitig sehr dünn besiedelt. Sie scheinen daher beliebte Ausflugsziele für Geocacher zu sein.

\section{Zusammenfassung und Ausblick}

Diese Arbeit ist ein wissenschaftlicher Beitrag im Bereich von nutzergenerierten raumbezogenen Daten für den weltweit beliebten Sport Geocaching. Zugleich wurde das Vorgehen speziell für Anwendungen in der Lehre entwickelt und beinhaltet einen didaktischen Ansatz zur Vermittlung fortgeschrittener Kenntnisse in den Bereichen GIS, Geoprozessierung, offenen Geodaten und Data Science. Aufgrund der offenen Verfügbarkeit von Daten und Software sowie ausführlichen Anleitungen und Lernmaterial auf der OpenGeoEdu-Webseite ist die Arbeit im hohen Maße reproduzierbar.

In fachlicher wie in didaktischer Hinsicht ist die Versatilität des hier vorgestellten Ansatzes eine seiner Stärken. Es ist vergleichsweise einfach, weitere Betrachtungen darauf aufzubauen und das Vorgehen auf unterschiedliche Regionen und Länder zu übertragen. Dazu sind i) Forschende, die weitere Erkenntnisse aus dem Bereich nutzergenerierter Geodaten ergründen, ii) Lernende, die sich für das entdeckende Lernen begeistern, und iii) Lehrende, die die vorliegende Lerneinheit in ihre Lehre integrieren, ausdrücklich eingeladen.

\section{Danksagung}

Der Verfasser dankt dem Bundesministerium für Verkehr und Digitale Infrastruktur (BMVI) für die Förderung im Rahmen des mFUND-Programms (FKZ: 19F2007A).

\section{Literatur}

Cord, A. F., Roeßiger, F., Schwarz, N. (2015): Geocaching data as an indicator for recreational ecosystem services in urban areas: Exploring spatial gradients, preferences and motivations, Landscape and Urban Planning, Ausgabe 144, 151-162. doi:10.1016/j.landurbplan.2015.08.015

Golbeck, J., Neustaedter, C. (2016): Environmental Factors Affecting Where People Geocache, Future Internet, MDPI AG, Ausgabe 8/2, 11. doi:10.3390/fi8020011

Hödl, C., Pröbstl-Haider, U. (2017): Geocaching in Austrian National Parks, eco.mont Journal on Protected Mountain Areas Research, Verlag der Österreichischen 
Akademie der Wissenschaften, Ausgabe 9/2, 42-51. doi:10.1553/eco.mont-92s 42

Mendes, R. N., Rodrigues, T., Rodrigues, A. M. (2013): Urban geocaching. What happened in Lisbon during the last decade, ISPRS - International Archives of the Photogrammetry, Remote Sensing and Spatial Information Sciences, Copernicus $\mathrm{GmbH}$, Ausgabe XL-4/W1, 29-31.

O'Hara, K. (2008): Understanding geocaching practices and motivations, Proceedings of the SIGCHI conference on human factors in computing systems, ACM Press, 1177-1186.doi: 10.1145/1357054.1357239

Rosário, I. T., Rebelo, R., Cardoso, P., Segurado, P., Mendes, R. N., Santos-Reis, M. (2019): Can geocaching be an indicator of cultural ecosystem services? The case of the montado savannah-like landscape, Ecological Indicators, Elsevier, Ausgabe 99, 375-386. doi:10.1016/j.ecolind.2018.12.003

Santos, T., Mendes, R. N., Rodrigues, A. M., Freire, S. (2012): Treasure Hunting in the 21st century: A Decade of Geocaching in Portugal, Nagle T.(éd.), 6th European Conference on Information Management and Evaluation (ECIME 2012), Cork, Academic Conferences and Publishing International, 273-281.

Weber, K., Haug, S. (2012): Geocaching und Raumnutzung, Standort, Springer Berlin Heidelberg, Ausgabe 36, 17-24. doi:10.1007/s00548-012-0195-3 
Virtuelle Welten 



\title{
Virtuelle Touren im Kontext aktueller Mixed-Reality-Technologien
}

Markus Berger, Sarah Westphal, Patrick Postert, Hannes Hagen

Universität Rostock

\{markus.berger|sarah.westphal|patrick.postert|hannes.hagen\}@uni-rostock.de

\begin{abstract}
Jederzeit einen Blick auf weit entferne Orte werfen zu können ist durch Webdienste wie Google StreetView inzwischen fast eine Selbstverständlichkeit. So ist es nicht verwunderlich, dass Akteure von Kulturstätten bis hin zu Unternehmen sich auf diese Weise präsentieren wollen, um interessierten Nutzergruppen Einblicke in bestimmte Umgebungen zu gewähren. Dies kann sowohl zum Präsentieren eines IstZustandes als auch für Blicke in die Vergangenheit und Zukunft genutzt werden. Besonders naheliegend ist dabei die Verbindung mit immersiven Technologien wie Augmented und Virtual Reality, da diese einen Sinn von Räumlichkeit anbieten, der auf herkömmlichen Computersystemen nicht erreicht werden kann. In diesem Artikel werden aktuelle Technologien, Entwicklungen und Möglichkeiten im Bereich dieser immersiven virtuellen Touren zusammengetragen und am Beispiel vorgestellt.
\end{abstract}

\section{$1 \quad$ Einleitung}

Da der Begriff der virtuellen Tour in so vielen verschiedenen Kontexten verwendet wird, bleibt er meist schwach definiert. Er reicht von mit Informationen angereicherten Bilderstrecken hin zu vollumfänglich gerenderten, multisensorischen Erlebnissen. Im Laufe dieser Arbeit soll speziell auf die Arten von virtuellen Touren geschaut werden, bei denen immersive Technologien wie Augmented Reality (AR) und Virtual Reality (VR) zum Einsatz kommen. Unter „Immersion“ versteht Slater et al. (Slater, Usoh, \& Steed, 1995) ein objektives Maß dafür, inwieweit ein Display-System die Sinne des Nutzers einnimmt und mit digitaler Information überlagert. AR und VR reihen sich dabei auf dem sogenannten Mixed-Reality-Kontinuum ein, welches nach Milgram et al. (Milgram \& Kishino, 1994) von einer komplett realen Umgebung (Reality) bis hin zu einer perfekt überzeugenden virtuellen Welt (Virtuality) reicht. 
Immersive virtuelle Touren sind für diesen Artikel definiert als jene Anwendungen, in denen das virtuelle Ende des Kontinuums eine real existierende Umgebung repräsentiert. Im Fokus steht dabei die derzeitige technologische Situation beim Entwerfen und Veröffentlichen solcher Touren. Am Ende dieses Artikels sollten Leser einen guten Eindruck davon haben, mit welchen Mitteln sie eine virtuelle Tour für ein spezifisches Anwendungsgebiet umsetzen können.

\section{Faktoren}

\subsection{Immersion}

Die grundlegendste Entscheidung vor der Entwicklung einer Tour ist der gewünschte Grad an Immersion. Dabei sind vor allem zwei Aussagen zu treffen: VR eignet sich für Orte, die schwer oder gar nicht erreichbar sind oder für die aus der Ferne Werbung gemacht werden soll. AR eignet sich vor allem für Situationen, in denen das reale Erlebnis vor Ort aufgewertet werden soll, z. B. durch zusätzliche Inhalte oder Wegbeschreibungen. Beide Technologien können auch für die jeweils andere Anwendung verwendet werden, werden dort jedoch im Allgemeinen weniger effektiv sein. Bei bestimmten Touren, insbesondere in VR, sollte außerdem auch auf das Maß der „Präsenz“ geachtet werden. Dieses beschreibt den subjektiven Eindruck in einer virtuellen Umgebung, ,,vor Ort“ zu sein. (Slater, Usoh, \& Steed, 1995)

\subsection{Hardware}

Sobald die Zielsetzung der Tour klar ist, muss über die benötigte Hardware nachgedacht werden. Zuerst ist dabei die Display-Hardware wichtig. Hierbei wird unterschieden zwischen Smartphones bzw. Tablets, Head-Mounted Displays (HMDs) und projektorbasierten Systemen. Smartphones bieten den geringsten Grad an Immersion, können aber zumindest AR ohne zusätzliche Hardware anbieten. Setzt man stattdessen auf HMDs muss man diese entweder vor Ort bereitstellen oder akzeptieren, dass man nur einen kleinen Anwenderkreis erreichen wird. Am stärksten spezialisiert sind schlussendlich Projektorbasierte Systeme. Diese haben die Eigenheit, dass sie in den echten Raum integriert werden müssen - entweder müssen sie perfekt auf bestimmte Objekte eingestellt sein (AR) oder sie werden mit Leinwänden als CAVE-System betrieben (VR).

Neben der Displaytechnologie stellt sich auf Entwicklerseite die Frage nach Aufnahmehardware, meistens im Falle von VR-Touren. Will man fotorealistische Repräsentationen von bestimmten Orten anbieten, dann ist es derzeit nahe- 
zu unmöglich, dies über Echtzeit-Rendering zu erreichen. Stattdessen müssen für immersive Umgebungen passende Aufnahmen gemacht werden. Möglichkeiten sind hier Panoramen, Stereo-Panoramen und volumetrische Aufnahmen. Einfache Panoramen können mittels herkömmlicher Smartphones unter Einsatz entsprechender Apps erreicht werden, Stereo-Panoramen erfordern zumindest ein Zweikamerasystem. Egal wie ein Panorama jedoch aufgenommen wird, der Blickpunkt bleibt statisch. Soll der Nutzer sich frei in sechs Freiheitsgraden durch die Umgebung bewegen können, muss diese volumetrisch aufgenommen werden. Für einzelne Objekte ist dies mit einer herkömmlichen Kamera und entsprechender Software möglich, für größere Bereiche sind Spezialgeräte wie z. B. Laserscanner erforderlich. Eine andere Möglichkeit ist das stilisierte Rendering in nicht-fotorealistischer Ästhetik. Dies ist besonders dann gut realisierbar, wenn 3D-Daten bereits vorliegen.

\subsection{Software}

Eng verwoben mit der Wahl der Hardware ist die Softwaregrundlage der geplanten virtuellen Tour. Software beschreibt an dieser Stelle vor allem die Art, wie auf diese Tour vom Nutzer zugegriffen werden kann; aber auch die verfügbaren Entwicklungswerkzeuge spielen eine Rolle. Die erste Möglichkeit sind web-basierte Lösungen. Diese sind vor allem dann lohnenswert, wenn die Nutzer die Tour auf ihrer eigenen Hardware erleben sollen, da die Standardisierung hier weit fortgeschritten ist und fast jeder verbreitete Browser die entsprechenden Web-Schnittstellen beherrscht. Mit Frameworks wie A-Frame ist auch auf Entwicklerseite eine gute Unterstützung vorhanden. Erfordert eine Tour jedoch besondere Hardware oder hohe Performance, sind Stand-alone-Lösungen anstatt Web-Anwendungen empfehlenswert. Auch diese sollten in den meisten Fällen mit fertigen Werkzeugen wie z. B. der Unity oder der Spiel-Engine Unreal erstellt werden, da eine komplette Eigenentwicklung für VR oder AR oft mehr Fallstricke mit sich bringt als herkömmliche Desktop-Software (wie z. B. die Leserlichkeit von Text und die hohe Heterogenität bei Controllern).

\subsection{Datengrundlage \& Inhalte}

Eines der größten Hindernisse beim Erstellen von virtuellen Touren ist die Verfügbarkeit von Informationen über die Umgebung. Im Idealfall liegt ein genaues Modell der Umgebung vor, wie z. B. BIM oder CityGML. Ist dies nicht gegeben, stellen sich verschiedene Probleme: Will man z. B. eine Abfolge an Panorama-Bildern zeigen, dann muss per Augenmaß abgeschätzt werden, wo im Panorama bestimmte Inhalte sichtbar sind. Auch in der Umgebung angezeigte AR-Inhalte müssten entweder durch wiederholtes Ausprobieren oder (technisch aufwendig) dynamisch platziert werden. Aber auch bereits vorhandene 
Datensätze müssen weiterverarbeitet werden, da die in ComputergrafikAnwendungen verwendeten Koordinatensysteme und geometrischen Modelle wesentliche Unterschiede zu denen aus der Geoinformatik aufweisen. Oft ist hier das Überführen in Austauschformate wie FBX nötig.

\subsection{Interaktionen \& Darstellung}

Die vorangegangenen vier Punkte sind hinreichend um eine virtuelle Tour umzusetzen. Es gibt jedoch noch weitere qualitative Faktoren. Vor allem in VR stellt sich die Frage der virtuellen Nutzerbewegung, auch genannt „Locomotion". Die am häufigsten angewendeten Methoden sind Joystick-gesteuerte Bewegung und Teleportation (Bozgeyikli, Raij, Katkoori, \& Dubey, 2016). Meist gibt die Art der Datenaufnahme die Locomotion-Methode vor - zwischen statischen Panorama-Fotos ist keine kontinuierliche Bewegung möglich, daher bleibt nur eine Teleportation -, oft schränkt aber auch die Interaktionsperipherie der Nutzer ein.

In AR wiederum ist die Verdeckung von großer Bedeutung. Hier muss nicht nur die Verdeckung zwischen virtuellen Objekten behandelt werden, sondern auch die Verdeckung dieser Objekte durch die echte Welt und viceversa. Ist das nicht möglich, muss die Tour zur Vermeidung von Tiefenkonflikten räumlich stark eingegrenzt werden. Ein verwandtes Problem ist die Lokalisierung. Zwar ist das kontinuierliche Tracking über Technologien wie ARCore und ARKit mit relativ wenig Drift möglich, die Erstpositionierung ist jedoch nach wie vor problematisch. Die Genauigkeit von GNSS in mobilen Endgeräten ist i. d. R. beschränkt und eignet sich nicht für räumlich exakt platzierte Inhalte, während visuelle Erstpositionierung derzeit nur über fest in der Umgebung installierte Marker möglich ist.

\section{$3 \quad$ Beispiele}

\subsection{VR-Tour Botanischer Garten}

Der Botanische Garten Rostock ist gleichzeitig als Forschungs- und Freizeitumgebung konzipiert. Wer über das ausgedehnte Wegenetz wandert, wird dies zumeist tun, ohne über ausgiebiges Hintergrundwissen zu verfügen. Um Nutzern einen Blick auf genau solche Informationen zu bieten, wurde eine VR-Tour für den Garten konzipiert. Die benötigten Inhalte sind technisch eher unkompliziert und nur bestimmte Bereiche (Beet- und Biotopanlagen) sind von Interesse. Stattdessen stand Verfügbarkeit im Vordergrund. Es wurden PanoramaAufnahmen von diesen Bereichen erstellt und mittels A-Frame- 
Standardkomponenten verbunden. Dadurch ist die Tour auf jedem modernen Endgerät mit entsprechendem Webbrowser benutzbar, von Desktop-Systemen bis hin zu HMDs. Anlagebereiche wurden mit $360^{\circ}$-Panoramen aufgenommen (siehe Abbildung 1c), Objekte von speziellem Interesse als $180^{\circ}$ Stereopanoramen.
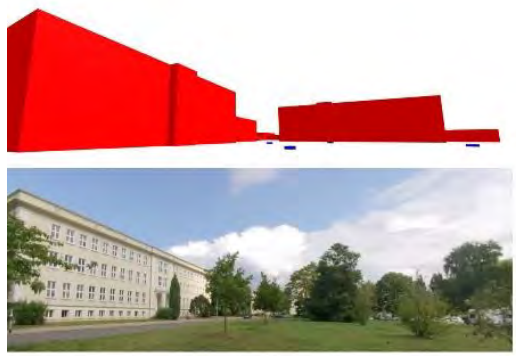

(a)

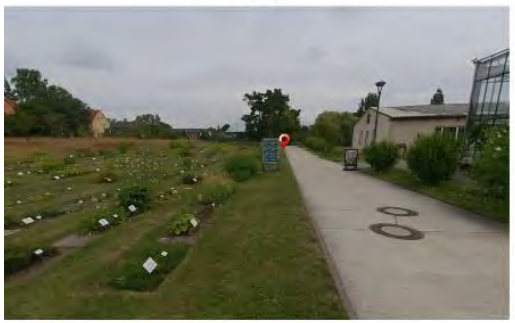

(c)

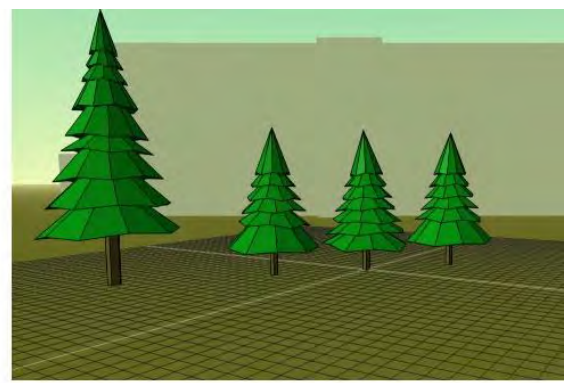

(b)

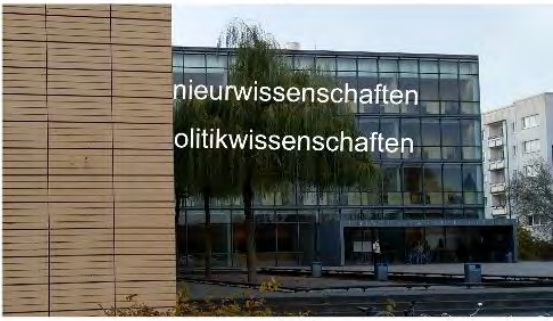

(d)

Abbildung 1: (a) Sichtachsen aus OSM-Geometrie, (b) prozedurale Bäume, (c) Panorama-Aufnahme mit Sprungpunkt, (d) AR-Sichtachsen

\subsection{VR-Führung Campus}

Für den AUF-Campus in der Rostocker Südstadt sollte eine Tour erstellt werden, auf der Nutzer von zu Hause den Campus erkunden und die verschiedenen Gebäude kennenlernen können. Zum einen stand also auch hier die Verfügbarkeit im Vordergrund, zum anderen sollen Nutzer ein Verständnis der räumlichen Zusammenhänge bekommen. Dies ist bei statischen Bildern oft problematisch, daher wurde ein hybrider Ansatz gewählt. Zuerst wird Nutzern eine kontinuierliche Bewegung durch ein komplett virtuelles Abbild des Campus ermöglicht. Dieses wird mithilfe von OpenStreetMap (OSM) Daten in einer A-Frame Szene in einem „Low-Poly“-Stil prozedural gerendert (siehe Abbildung 1b). An bestimmten Stellen auf dem virtuellen Gelände kann dann über eine einfache In- 
teraktion ein Blick in die reale Welt in Form von $180^{\circ}$-Stereopanoramen geworfen werden. Diese wurden mit hochgenauem GNSS eingemessen. Zusätzliche Inhalte wie z. B. Gebäude-Labels können in beiden Betrachtungsmodi weitestgehend ohne Änderung angezeigt werden, da die Sichtachsen abgesehen von Baumbewuchs gut übereinstimmen (siehe Abbildung 1a).

\subsection{AR-Führung in Stadtgebiet}

Aufbauend darauf folgten Überlegungen für eine Anwendung, welche nicht nur Gebäudeinformationen auf einem vorher festgelegten virtuellen Gelände anzeigen kann, sondern auf mobilen Endgeräten in beliebigen urbanen Umgebungen. $\mathrm{Da}$ für eine korrekte Verdeckung durch Gebäudegeometrie die derzeitige Sichtachse genau bekannt sein muss, ist die Erstpositionierung ein wichtiges Problem. Hier bleibt derzeit nur die Arbeit mit sogenannten Image Targets - gut erkennbaren Bildern, die im gewünschten Gelände platziert werden müssen. Im Umkreis dieses Targets können dann Gebäudedaten z. B. aus OSM oder aus einem vorhandenen CityGML-Datensatz geladen werden. Die Geometrien werden sowohl genutzt, um Verdeckungen abzubilden, als auch, um Annotationen und Gebäudeinformationen visuell auszurichten (siehe Abbildung 1d). An dieser Stelle wurde anstatt von Web-Technologien die Unity Engine mit ihrem ARFoundation Framework genutzt, da eine Standalone-App besser dafür geeignet ist, gleichzeitig das Laden und Verarbeiten von Daten, das Rendern von Informationen und die notwendigen Computer-Vision Prozesse mit guter Performance ablaufen zu lassen.

\section{$4 \quad$ Zusammenfassung und Ausblick}

In Anbetracht all dieser Möglichkeiten ergibt sich die Schlussfolgerung, dass sich die technische Basis für immersive virtuelle Touren heutzutage in einem guten Zustand befindet. Besonders im Bereich VR ist eine hervorragende technische Unterstützung gegeben - sogar ganz ohne Programmierkenntnisse können ansehnliche Touren erstellt werden. Für AR bestehen weiterhin Probleme mit Display-Systemen und der Erstpositionierung, welche aber nicht nur virtuelle Touren, sondern das gesamte Segment betreffen. Hier ist in den nächsten Jahren mit großen Verbesserungen zu rechnen. 


\section{Literatur}

Bozgeyikli, E., Raij, A., Katkoori, S., Dubey, R. (2016). Point \& Teleport Locomotion Technique for Virtual Reality. Proceedings of the 2016 Annual Symposium on Computer-Human Interaction in Play, (S. 205-216).

Milgram, P., Kishino, F. (1994). A taxonomy of mixed reality visual displays. IEICE TRANSACTIONS on Information and Systems 77 (12), S. 1321-1329.

Slater, M., Usoh, M., Steed, A. (1995). Taking steps: the influence of a walking technique on presence in virtual reality. ACM Transactions on ComputerHuman Interaction (TOCHI), S. 201-219. 



\title{
Virtueller Zwilling für ein dynamisches Lagermanagement in Häfen
}

\author{
Olaf Poenicke ${ }^{1}$, Andreas Höpfner ${ }^{1}$, Christian Blobner ${ }^{1}$, Heiko Maly ${ }^{2}$ \\ ${ }^{1}$ Fraunhofer IFF, Sandtorstraße 22, 39106 Magdeburg \\ ${ }^{2}$ Transportwerk Magdeburger Hafen GmbH, Saalestraße 20, 39126 Magdeburg
}

\begin{abstract}
Im Rahmen des Projekts PortForward wurden bisher die technischen Konzepte und Grundlagen erarbeitet, um auf Basis von Echtzeit3D-Informationen ein dynamisches Lagermanagement aufbauen zu können. Mit dem realen Abbild der Lagersituation im für verschiedenste Güterarten genutzten Hanseterminal, können Potenziale zur Optimierung des Lagermanagements erschlossen werden. Im weiteren Projektverlauf werden die technischen Installationen im Hafen vorgenommen und die Funktionen zur Überführung der Scan-Daten in das 3D-Modell weiterentwickelt und erprobt.
\end{abstract}

\section{$1 \quad$ Einleitung}

Digitalisierung und Industrie 4.0 sind Schlagworte, die Unternehmen und Infrastrukturbetreiber vor neue Herausforderungen stellen. Die Fähigkeit, digitale Daten zu verarbeiten und vorzuhalten sowie Entscheidungen auf Basis digitaler Daten zu treffen, wird zum elementaren Bestandteil von Unternehmen, um ihre Wettbewerbsfähigkeit zu sichern. Dies ist umso wichtiger in der Logistik, die an den Knotenpunkten der Wirtschaft agiert.

Im Zuge der Digitalisierung stehen Häfen vor der Herausforderung, verschiedenste Daten und Informationen zu weit verteilten Infrastrukturen, Suprastrukturen, Betriebsmitteln sowie Fracht- und Lagergütern in integrierter Form intuitiv wahrnehmbar abzubilden. Das von der EU-Kommission im Rahmen des Horizont 2020 geförderte Projekt PortForward (2018-2021) setzt an diesen Punkten und Herausforderungen im Hinblick auf die Digitalisierung von Hafeninfrastrukturen an. Das Fraunhofer IFF leitet das Projekt und arbeitet mit zwölf europäischen Partnern an der Entwicklung von Lösungen und Technologien für kleine und mittelgroße Häfen in Europa. Das IFF setzt im PortForwardProjekt vor allem auf seine langjährige Kooperation mit dem Magdeburger 
Hafen. Hier erfolgte in Vorprojekten bereits die Erstellung virtueller Modelle zur Infrastrukturplanung. Im Rahmen von PortForward entwickelt das Fraunhofer IFF vor diesem Hintergrund einen sogenannten Virtuellen Zwilling des Binnenhafens Magdeburg.

\section{Virtueller Zwilling des Hafens}

Der Ansatz des Virtuellen Zwillings fußt auf dem Konzept des Digitalen Zwillings, welches erstmalig in (Shafto et al., 2010) vorgestellt wurde. Mit dem Aufkommen neuer und leistungsfähiger Virtual-Reality(VR)-Technologien verlagert sich der Schwerpunkt dieses Konzepts mehr auf die virtuellen und interaktiven Darstellungen der Digital-Twin-Lösungen (vgl. Schroeder et al., 2016).

Durch das Fraunhofer IFF werden Lösungen des Virtuellen Zwillings durch die Integration verschiedener Systemmodelle erstellt. So wird zum Beispiel durch Höpfner et al. die Kombination eines VR-Raummodells mit einem EnergieModell von Industrieparks beschrieben (Höpfner et al., 2017). Für die Anwendung im Hafenumfeld steht vor allem die Kombination des Raummodells mit einem Logistikmodell des Hafens im Fokus.

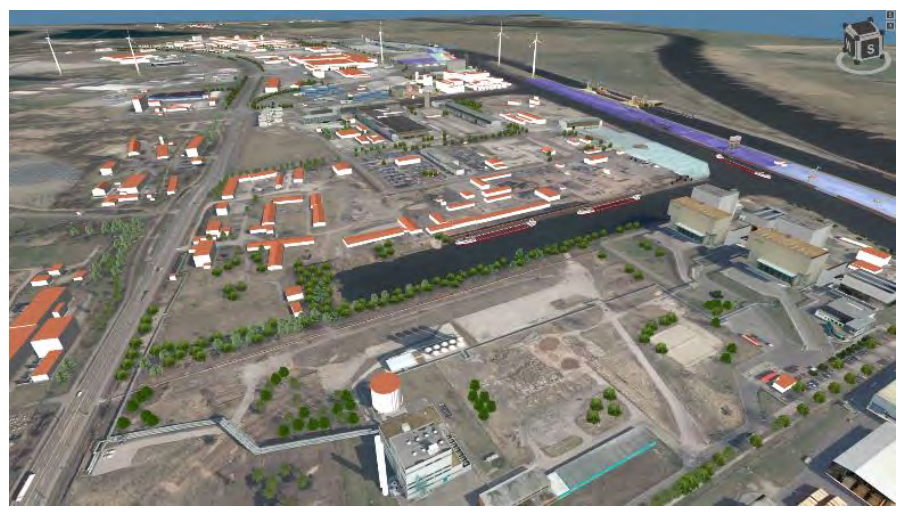

Abbildung 1: Ausschnitt des statischen Raummodells des Magdeburger Hafens

Zur Generierung des statischen 3D-Modells wurden Geobasisdaten des Landesamtes für Vermessung und Geoinformatik LVermGeo des Landes SachsenAnhalt herangezogen. Ausgangsdaten zur Generierung des Geländemodells war das in Sachsen-Anhalt angebotene Digitale Geländemodell DGM 02 (Standard- 
Gitterweite 2,0 m; Genauigkeit der Höhe +/- 0,15 m). Weitere Ausgangsdaten zur Geländemodellerstellung waren Digitale Orthofotos in einer Auflösung von $20 \mathrm{~cm} /$ Pixel DOP20. Diese Geobasisdaten wurden in einem ersten Schritt in die VRS-Plattform des Fraunhofer IFF importiert, sodass ein vorläufiges Geländemodell generiert werden konnte.

Die besonderen Anforderungen der späteren Modellanwendung machten eine weitere Bearbeitung dieses Modells erforderlich. Die entstandene Geländegeometrie des bisher regelmäßigen DGM wurde in ein volumen- und performanceoptimiertes unregelmäßiges Dreiecksnetz TIN (Triangulated Irregular Network) überführt. Die Terminalflächen des Hafens Magdeburg wurden auf Basis von digitalen Plänen manuell integriert. Uferbebauungen und Hafeninfrastrukturen, wie Kaimauern und Schleusenanlagen wurden über Bestandspläne des Magdeburger Hafens und über Vor-Ort-Fotoaufnahmen in das Geländemodell integriert. Im Ergebnis entstand ein virtuelles 3D-Geländemodell des Magdeburger Hafens, welches sich für die interaktive Arbeit in Echtzeit eignet. In einem nächsten Schritt wurden vom LVermGeo Sachsen-Anhalt angebotene Level-ofDetail-02-Gebäude in die VRS-Plattform des Fraunhofer IFF importiert und als zweite Modellkomponente mit dem bestehenden, optimierten Geländemodell zusammengeführt. Ausgewählte Level-of-Detail-02-Gebäude von besonderer Relevanz wurden mit Fassadentexturen versehen (vgl. Abbildung 2).
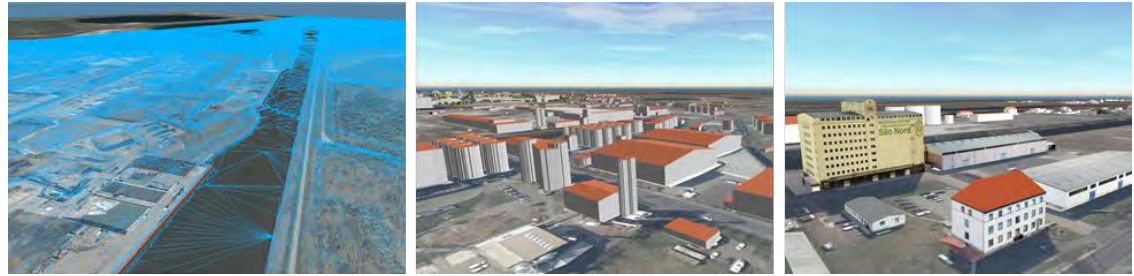

Abbildung 2: Geländemodell mit 3D-Geometriekanten (links); 3D-Gebäudemodelle mit LoD 2 (Mitte); texturierte Gebäudemodelle (rechts)

In einem abschließenden Modellierungsschritt wurden stadtbildprägende Vegetationen und sekundäre Objekte manuell über eine 3D-Objektbibliothek in das virtuelle Modell integriert, sodass im Ergebnis ein geeignetes 3D-Gesamtmodell für die weitere Erarbeitung des dynamischen Lagermanagements zur Verfügung steht. 


\section{Anforderungen an ein dynamisches Lagermanagement}

Im Magdeburger Hafen werden einzelne Hafenterminals für den Umschlag und die Lagerung unterschiedlichster Güter genutzt (z. B. Mischnutzung mit palettierter Ware, Containern, Schwerlastteilen etc.). Aufgrund dessen lassen sich keine festen Lagerplätze definieren und im Terminalbereich markieren, wie es beispielsweise in reinen Containerterminals mit Markierung von Lagergassen und Stellplätzen erfolgt. Unter dieser Prämisse wurde der Ansatz für ein virtuelles Lagerplatz-Raster entwickelt, für das das Raummodell des Hafens als Grundlage dient. Mit einem Rastermaß von 2x2 Metern lassen sich somit eingelagerte Güter einzelnen virtuellen Lagerplätzen zuordnen.

Diese Zuordnung macht eine Erfassung der Güterinformationen und Verortung bei Einlagerung erforderlich. Im Projekt soll diese Erfassung mit Unterstützung mobiler Endgeräte erfolgen, sodass während der Einlagerung eine grobe Positionserfassung über integrierte GPS-Sensorik erfolgt. Für ein dynamisches Lagermanagement ist diese Positionserfassung aber zu ungenau, sodass eine sensorische Unterstützung durch Kameratechnologie erfolgt. Im fokussierten HanseTerminal des Magdeburger Hafens besteht bereits eine Kamerainfrastruktur für Forschungszwecke, mit der eine sogenannte Virtuelle Draufsicht (siehe Abbildung 3) (Borstell et al., 2012) generiert werden kann. Diese ermöglicht auf Basis von Differenzbildanalysen eine Erfassung der Belegung der einzelnen Lagerraster.

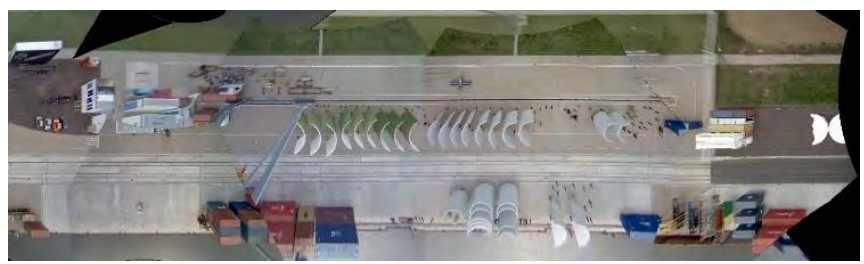

Abbildung 3: Virtuelle Draufsicht auf das Hanse-Terminal

Um für den Virtuellen Zwilling auch 3D-Informationen zur aktuellen Lagerbelegung zu gewinnen, wird aktuell ein Ansatz erarbeitet, mittels im Hafen installierter LiDAR-Sensoren Realkonturen aufzunehmen und in abstrahierter Form in das 3D-Modell des Hafens zu integrieren. 


\section{$4 \quad$ Erfassung des Lagerzustands durch LiDAR-Sensorik}

Light-Detection-and-Ranging(LiDAR)-Sensoren gehören zu der Gruppe der Time-of-Flight-Sensoren, die Entfernungen ermitteln, indem Licht emittiert wird und die Entfernungen durch das Messen der Flugzeit des Lichts, das an Oberflächen reflektiert und wieder zum Sensor zurückgestrahlt wird, gemessen wird. Da der LiDAR-Sensor einen Laserstrahl in eine vorher bekannte Richtung emittiert, kann dadurch eine 3D-Koordinate im Raum relativ zur Position des Sensors bestimmt werden. Über den Zeitverlauf entstehen somit 3DPunktewolken, die sich nutzen lassen, um räumliche Abbilder zu erzeugen und Bewegungen zu detektieren und zu lokalisieren. LiDAR-Sensoren kommen vor allem im Kontext des autonomen Fahrens zum Einsatz, da sie hohe Lesereichweiten von $>>100 \mathrm{~m}$ bieten und die 3D-Daten vergleichsweise schnell zur Verfügung stellen. Am Markt sind erste Modelle auch im Low-Cost-Bereich von $<1.000 €$ verfügbar, wodurch sich neue Anwendungsfelder erschließen lassen.

Für die oben beschriebene Zielanwendung bietet das von der Firma Livox verfügbare Modell MID-40 ein sehr gutes Preis-Leistungsverhältnis. Die von Ortiz Arteaga et al. analysierten Leistungsparameter weisen eine sehr hohe Genauigkeit des Sensors bei Messreichweiten bis über $200 \mathrm{~m}$ auf (vgl. Ortiz Arteaga et al., 2019). Die Besonderheit des Sensors liegt in einem non-repetitive Scanning Pattern, wodurch sich gegenüber zeilenbasierten LiDAR-Sensoren eine höhere Abdeckung des Field of View (FOV) erzielen lässt (Livox, 2020), was insbesondere auch für die Erfassung von 3D-Objekten im Hafenterminal über einen längeren Zeitraum verbessert. In Abbildung 4 sind exemplarische Testaufnahmen mit dem LiDAR-Sensor im Hanse-Terminal Magdeburg abgebildet.

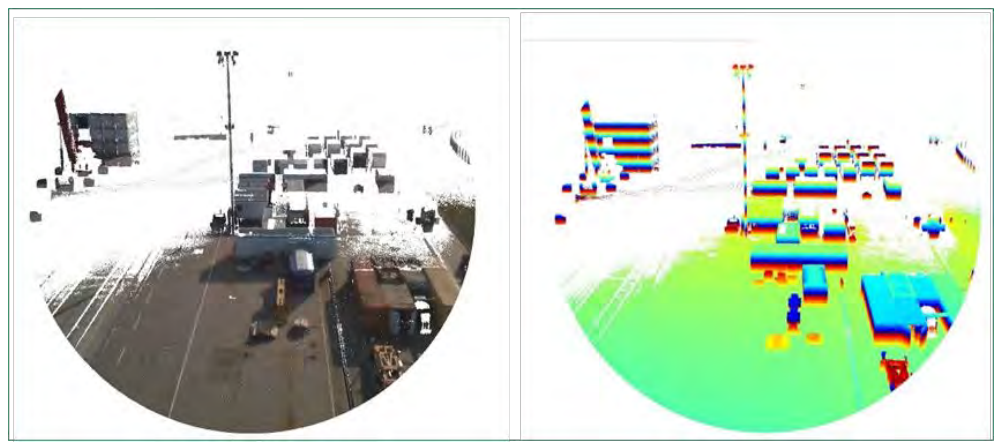

Abbildung 4: LiDAR-Aufnahme mit RGB-Mapping (links) und mit Höhencodierung (rechts) 
Aktuell wird eine Kameraplanung umgesetzt, um durch Ausstattung mit LiDAR-Sensoren an den Lichtmasten im Hanse-Terminal definierte Lagerbereiche erfassen zu können. Die Installationen werden so geplant, dass ein Lagerbereich von mindestens zwei Seiten erfasst wird, um durch das Matching der Punktwolken die eingelagerten Objekte allseitig zu erfassen.

Auf Basis der generierten Punktwolken lassen sich Standardformen und -größen (z. B. für ISO-Container oder Wechselbrücken) klassifizieren und als abstrahiertes Objekt direkt in das 3D-Modell des Hafens integrieren. Fracht und Gebinde mit nicht direkt klassifizierbaren Formen werden mittels Bounding Box übergeben. Die Übergabe der Daten zu Objekttyp, Größe, Position, Orientierung und Grundfarbe vom Messsystem an das 3D-Modell erfolgt mithilfe des Machine2Machine-Kommunikationsprotokolls MQTT.

\section{Zusammenfassung und Ausblick}

Im Rahmen des Projekts PortForward wurden bisher die technischen Konzepte und Grundlagen erarbeitet, um auf Basis von Echtzeit-3D-Informationen ein dynamisches Lagermanagement aufbauen zu können. Mit dem realen Abbild der Lagersituation im für verschiedenste Güterarten genutzten Hanse-Terminal, können Potenziale zur Optimierung des Lagermanagements erschlossen werden. Im weiteren Projektverlauf werden die technischen Installationen im Hafen vorgenommen und die Funktionen zur Überführung der Scan-Daten in das 3DModell weiterentwickelt und erprobt.

Das Projekt PortForward wird von der EU unter der Projektnummer 769267 im Rahmen des Programms „Ports of the Future“ gefördert. https://www.portforward-project.eu/.

\section{Literatur}

Borstell, H., Plate, C., Richter, K. (2012): Virtuelle Draufsicht für die bildbasierte Situationsanalyse. Tagungsband InnoSecure 2012.

Hoepfner, A., Mencke, N., Lombardi, P., Franke, R., Komarnicki, P. (2017): A Virtual Reality Platform that supports integrated Design of Energy and Land-Use Plans in Brownfield Industrial Parks. The Seventh International Symposium on Energy. Manchester, 2017.

Livox Technology (2020): MID-40 lidar sensor - Livox. URL https://www.livoxtech.com/mid-40-and-mid100 (letzter Zugriff: 25.02.2020). 
Ortiz Arteaga, A., Scott, D., Boehm, J. (2019): Initial Investigation of a low-cost automotive LiDAR system. In: The International Archives of the Photogrammetry, Remote Sensing and Spatial Information Sciences, Volume XLII-2/W17.

Schroeder, G. N., Steinmetz, C., Pereira, C. E., Espindola, D. B. (2016): Digital Twin Data Modeling with Automation ML and a Communication Methodology for Data Exchange. In IFAC-PapersOnLine - Volume 49, Issue 30 (pp. 12-17). Laxenburg, Austria: International Federation of Automatic Control IFAC.

Shafto, M., Conroy, M. E. G., Kemp, C., Le Moigne, J., Wang, L. (2010): Draft modelling, simulation, information technology \& processing roadmap, Technology Area 11. Washington, DC: National Aeronautics and Space Administration. 



\title{
Helgoland 3D mit ArcGIS Pro
}

\author{
Stefan Liening \\ ARC-GREENLAB GmbH \\ liening.stefan@arc-greenlab.de
}

\begin{abstract}
Der Beitrag zeigt, welche umfassenden Möglichkeiten ArcGIS Pro bietet, um 3D-Daten zu visualisieren und zu bearbeiten. Im Fallbeispiel werden Gebäudedaten im Detaillierungsgrad 2 (LoD2) vom Kreis Pinneberg mit dem Höhenmodell des Landesamtes für Vermessung und Geoinformation Schleswig-Holstein kombiniert. Zum Kreis Pinneberg gehört unter anderem die Insel Helgoland, an deren Beispiel eindrucksvoll die Einsatzmöglichkeiten und Werkzeuge von ArcGIS Pro gezeigt werden können.
\end{abstract}

\section{$1 \quad$ Einleitung}

3D-Stadt- und Höhenmodelle bieten gegenüber 2D-Darstellungen diverse Vorteile und gewinnen zunehmend an Bedeutung. Sie können als Grundlage für weiterführende Analysen der städtebaulichen Situation sowie für die Stadtplanung und viele weitere Anwendungsbereiche genutzt werden. Die 3D-Modelle eignen sich neben der Beurteilung von Neubaumaßnahmen z. B. auch, um Sichtachsen oder Schattenwürfe geplanter Gebäude zu simulieren.

Sind die Modelle visuell ansprechend aufbereitet, können sie zudem für die Öffentlichkeitsarbeit, im Tourismus und zur Bürgerinformation genutzt werden. Die Esri Software ArcGIS Pro bietet mit den integrierten Werkzeugen vergleichsweise schnelle und einfache Möglichkeiten, optisch ansprechende, realitätsnahe 3D-Stadt- und Höhenmodelle $\mathrm{zu}$ modellieren, $\mathrm{zu}$ bearbeiten und $\mathrm{zu}$ visualisieren.

\section{Datengrundlage}

Die Datengrundlage bilden XML-Dateien. Diese Dateien kodieren die Informationen zu den Gebäuden (LoD2) im CityGML-Format. Mithilfe der Data In- 
teroperability Extension werden diese Daten eingelesen und dabei in eine FileGDB konvertiert. Die Daten sind in Streifen gemäß UTM-Gitter eingeteilt. Eine politische Unterteilung (Bundesland/Gemeinde/Kreis o. Ä.) gibt es nicht. Da nur Gebäude aus dem Kreis Pinneberg für das Projekt relevant sind, wurden diese aus dem Datensatz Schleswig-Holstein ausgeschnitten.

\section{Realistische Gebäude}

Um die Anschaulichkeit und Plastizität der Daten auf Helgoland zu verbessern, können verschiedene Symbolisierungen genutzt werden. So kann beispielsweise das gesamte Gebäudemodell eingefärbt werden. Alternativ stehen nach der Konvertierung der LoD2-Modelle auch die einzelnen Bestandteile der Gebäude zur Verfügung. Es ist also beispielsweise möglich, die Dächer oder Wände einzeln einzufärben.

Um die Darstellung noch realistischer zu gestalten, kam die Esri CityEngine zum Einsatz. Ein schneller Import der Daten aus der File-GDB ermöglicht die Bearbeitung der Gebäude in der Software. Mit dieser Software können allen Gebäudefassaden mit wenigen Klicks Texturen hinzugefügt werden. Diese Fassaden sind automatisch generiert und entsprechen nicht der Wirklichkeit - der optische Eindruck der Gebäude ist nun allerdings spürbar realitätsnaher (siehe Abbildung 1).

\section{Das Höhenmodell}

Zunächst wurde das in ArcGIS standardmäßig enthaltene Höhenmodell aus den World Elevation-Services von Esri für die Darstellung verwendet. Bei der Verwendung dieser globalen Sammlung von Höhendaten zeigte sich, dass es durch die großen Höhenunterschiede von Helgoland zu Problemen bei der Kollisionsabfrage kommen kann. Einige Gebäude fliegen in der Luft, andere werden vom Boden verschluckt. Um die Situation zu verbessern, wurde das genauere Höhenmodell des Landesamtes für Vermessung und Geoinformation SchleswigHolstein genutzt (siehe Abbildung 2). Damit stehen zwar alle Gebäude auf festem Boden, doch die Daten zeigen eine tiefe Verwerfung mitten auf Helgoland offensichtlich ein Ergebnis, dass noch einer Nachbearbeitung bedarf.

Hier bietet ArcGIS Pro passende Werkzeuge, um eine solche „Verwerfung“ digital zu bereinigen. In der Spatial Analyst Extension hat man Zugriff auf die Werkzeuge „Füllen“ und „Reklassifizieren“. „Füllen“ ermöglicht das Auffüllen 
von Senken in einem Oberflächenraster. Im Fall von Helgoland bleibt die Störung durch das Tool allerdings noch sichtbar. Um diese Absenkung vollends anzugleichen, wird das Tool „Reklassifizieren“ angewendet. Als Endergebnis erhält man eine störungsfreie Oberfläche ohne schwebende Häuser und unrealistische Schluchten. Im Anschluss kann das Projekt abgeschlossen und zur Veröffentlichung, beispielsweise als WebScene, freigeben werden.

\section{$5 \quad$ Beurteilung von Neubaumaßnahmen}

Im Kreis Pinneberg sollen die Daten vom Fachdienst Planen und Bauen genutzt werden. Dieser Fachdienst ist Ansprechpartner in Fragen des öffentlichen Baurechts und des Brandschutzes im Kreis Pinneberg und nimmt die Aufgaben der Bauaufsichtsbehörde für alle Kommunen des Kreises Pinneberg, mit Ausnahme der Städte Elmshorn, Pinneberg und Wedel wahr. Speziell soll das 3D-Modell zur Beurteilung von Neubaumaßnahmen herangezogen werden. Eine solche Neubaumaßnahme kann schnell in ArcGIS Pro simuliert werden, um zu überprüfen, wie sich Bauprojekte im 3D-Stadtmodell in die Umgebung einfügen (siehe Abbildung 3). Dazu können in ArcGIS Pro die 3D-Analyst-Tools genutzt werden. So lassen sich zum Beispiel Sichtachsen oder Schattenwürfe der geplanten Gebäude simulieren.

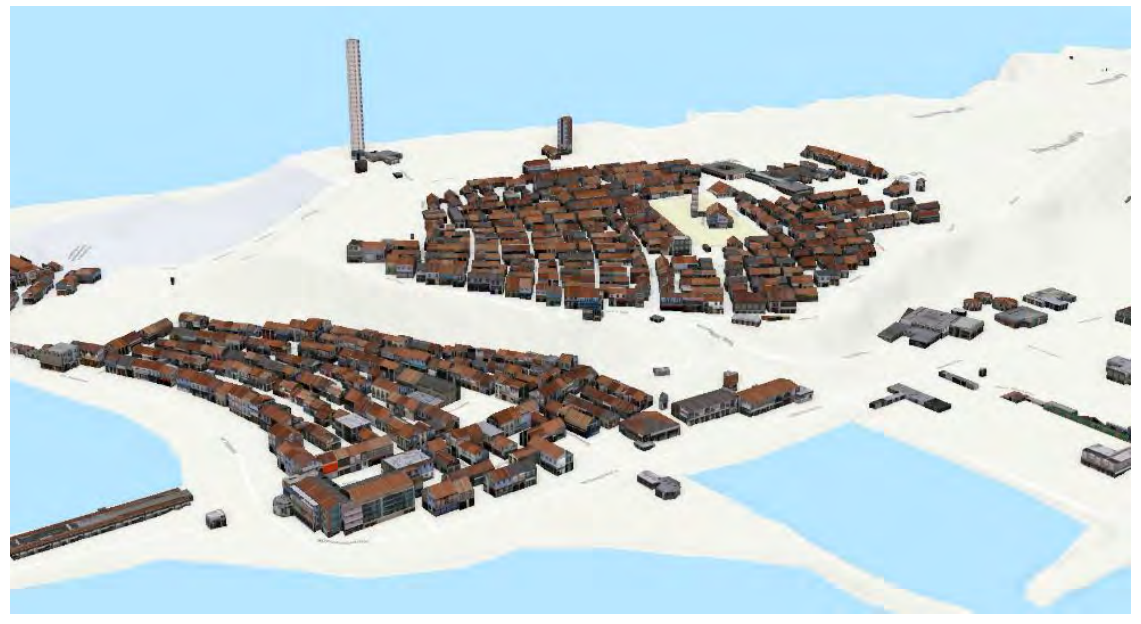

Abbildung 1: Insel Helgoland mit texturierten Gebäudemodellen 

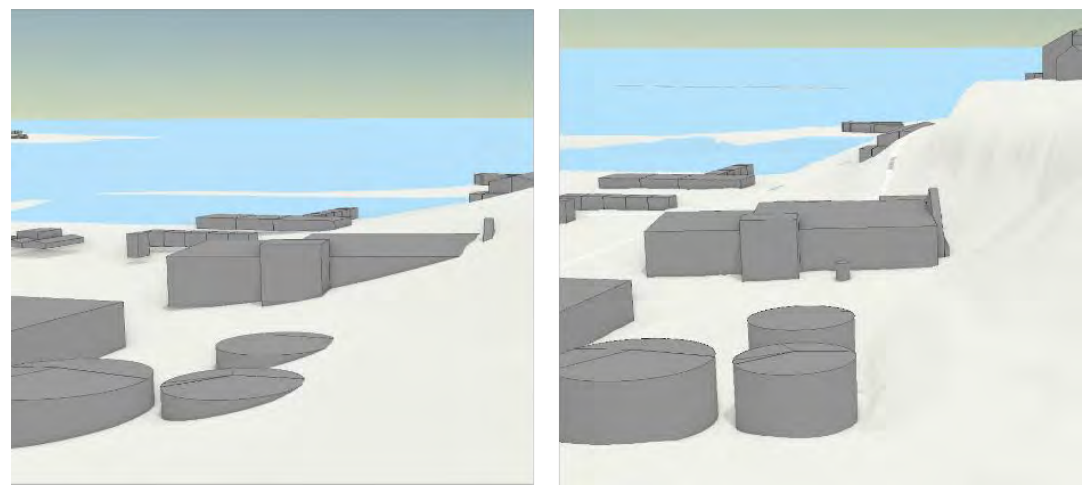

Abbildung 2: Vergleich der Höhenmodelle: World Elevation-Services (links), Landesamt für Vermessung und Geoinformation Schleswig-Holstein (rechts)

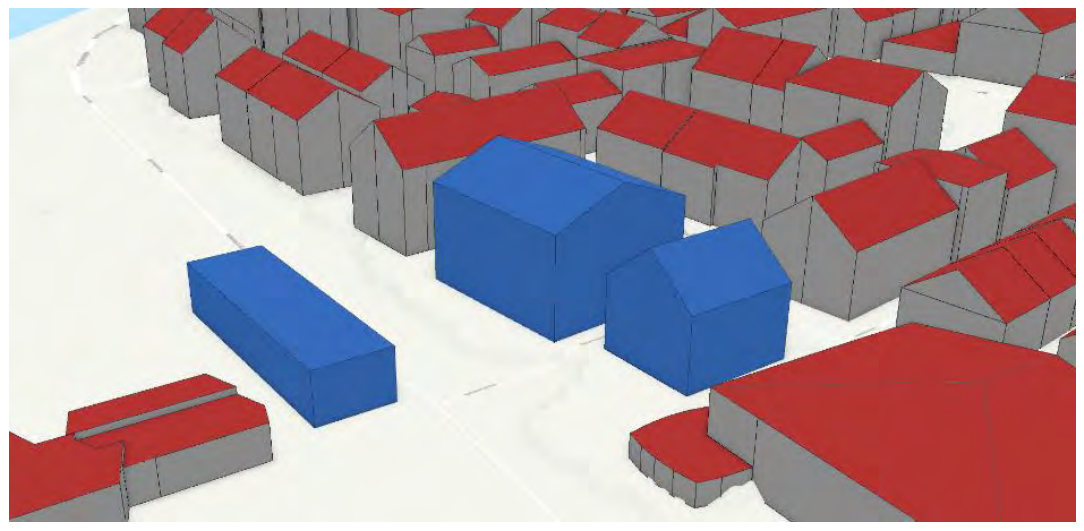

Abbildung 3: 3D-Modell zur Beurteilung von Neubaumaßnahmen

\section{$6 \quad$ Fazit}

Als Fazit kann festgehalten werden, dass das Visualisieren, Modellieren und Bearbeiten von 3D-Daten in ArcGIS Pro mit den integrierten Werkzeugen schnell und ohne großen Aufwand von der Hand geht. Als Ergebnis erhält man optisch ansprechende, realitätsnahe 3D-Stadt- und Höhenmodelle als Grundlage für weiterführende Analysen der städtebaulichen Situation sowie für die Stadtplanung und viele weitere Anwendungsbereiche. 


\section{Literatur}

Gebäudedaten und Höhenmodell vom Landesamt für Vermessung und Geoinformation Schleswig-Holstein, 2019 

Firmendarstellungen

\author{
AED-SICAD GmbH \\ ARC-GREENLAB GmbH \\ BeMaster GIS \\ CiS GmbH \\ CPA ReDev GmbH \\ DLR \\ DVZ Datenverarbeitungszentrum M-V GmbH \\ LAiV M-V
}



VertiGIS

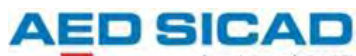

by VertiGIS
AED-SICAD by VertiGIS GmbH

12435 Berlin, Eichenstraße $3 b$

Telefon: 030/52000880, Fax: 030/520008811

E-Mail: holger.bronsch@aed-sicad.de

Internet: www.aed-sicad.de

\section{VERTIGIS MIT AED-SICAD ALS FÜHRENDEM UN- TERNEHMEN STELLT SICH VOR}

Die AED-SICAD GmbH ist ein führendes GIS-Applikationshaus in Europa, das Geschäftsprozessanalysen, Fach- und IT-Konzeptionen und Standardanwendungen für maßgeschneiderte Geo-Lösungen bietet. Es werden High-EndGeoinformationssysteme für die Kernsegmente Kataster und Landmanagement, die Ver- und Entsorgungswirtschaft, Kommunen und e-Government realisiert.

Der US-amerikanische Finanzinvestor Battery Ventures (BV) aus Waltham (Massachusetts) wird um AED-SICAD als Kern das neue Unternehmen „VertiGIS“ aufbauen. Dazu zählen die Geocom Informatik GmbH (D) und die Geocom AG $(\mathrm{CH})$, bisher Beteiligungen der Esri Deutschland $\mathrm{GmbH}$ und wie AED-SICAD Esri Platin Partner. Zusätzlich zählen der Partner SynerGIS GIS und FM Solutions (A) sowie dadurch die gemeinsame Tochter AEDSYNERGIS GmbH (D) zu VertiGIS; die Verschmelzung dieser Firmen ist in 2020 geplant. AED-SICAD besitzt darüber hinaus eine Mehrheitsbeteiligung an der BARAL Geohaus Consulting AG und ist mit einem Geschäftsanteil von $50 \%$ Gesellschafter der ARC-GREENLAB GmbH. Durch Standorte in München, Köln/Bonn, Berlin und Hamburg und Lösungspartner in ganz Deutschland wird ein hohes Maß an Kundennähe erreicht, auch werden die internationalen Vertriebskanäle von Esri und weiteren renommierten Partnerunternehmen genutzt.

Fachwissen und Marktkenntnis der hervorragend ausgebildeten Mitarbeiter des gesamten Unternehmens stehen unseren Kunden zur Verfügung; für dedizierte Anwendungen nutzen wir auch Open Source als Basis, damit bieten wir ein höchstes Maß an Investitionssicherheit. Insgesamt umfasst die Kundenbasis von AED-SICAD im In- und Ausland heute bereits deutlich über 1.000 Kunden. Wir sind strategischer Platin-Partner von Esri für die Bereiche Kataster/Landmanagement und Energieversorgungsunternehmen (EVU/Utilities). 
Die AED-SICAD GmbH (zukünftig VertiGIS) entwickelt wegweisende Lösungen für die Kernsegmente Landmanagement, Utility und Kommunal. Unser Produktportfolio für das Landmanagement umfasst Lösungen für Kataster und Landesvermessung (3A), die Flurbereinigung (LEFIS) und das Immobilien/Facility-Management (z. B. LISA). Die 3A-Produktlinie - die Komplettlösung für das Landmanagement als einer Schlüsselaufgabe der öffentlichen Verwaltung - beinhaltet alle zugehörigen Bereiche von AFIS ${ }^{\circledR}$, ALKIS ${ }^{\circledR}$ und ATKIS ${ }^{\circledR}$. Auf Basis dieser definierten Standards sind unsere Kunden für die Zukunft im Landmanagement bestens gewappnet.

Mit unseren umfassenden Utility-Lösungen für den Bereich Ver- und Entsorgung (UT for ArcGIS) sind wir auch international erfolgreich, nicht zuletzt durch enge Zusammenarbeit mit global aufgestellten Partnern wie Esri und SAP. AED-SICAD entwickelt schwerpunktmäßig auch Lösungen für die Verteilung (Web/Portale) und Langzeitspeicherung (LZS bis hin zur Archivierung) von Geo-Daten. Auf Basis von Dokument Management Systemen (DMS), z. B. unseres Partners SER GmbH als Kernsegment der LZS und unserem geospezifischen Lösungsanteil zur Langzeitspeicherung von Geodaten bieten wir dringend notwendige Langfristlösungen für Geo-Anwender und deren Geodatenspeicherung. Damit die mit unseren Lösungen erzeugten und verwalteten Geo-Daten auch den Weg zum Nutzer finden, haben wir alle Erfahrungen aus zahlreichen Projekten, z. B. von Daten-Migrationen in der Produktfamilie FUSION Data Service (FDS) als Datendrehscheibe gebündelt. Jetzt können die Daten in viele gängige Formate und Modelle konvertiert und somit zu neuen Informationsprodukten - auch INSPIRE-konform - aufbereitet werden.

\section{REFERENZEN}

Im Landmanagement mit hohem Anspruch an Sicherheit und Genauigkeit baut ein Großteil der zuständigen Verwaltungen und Dienstleister auf AED-SICAD 3A-Lösungen für den ALKIS-Katasterstandard, auf unsere ATKIS/AFIS- sowie unsere LEFIS-Lösungen. In der Ver- und Entsorgungswirtschaft wird das gesamte Spektrum vom international tätigen Multi-Utility-Konzern über regionale EVU und Flächenanbieter bis hin zu Stadtwerken und kommunalen Kanalbetreibern bedient. AED-SICAD kooperiert mit Universitäten und Museen. Lehrstühle in Geodäsie/Vermessung, Geoinformatik und Geographie nutzen Technologien von AED-SICAD für ihre Forschung, zur Umsetzung konkreter Anwendungsprojekte sowie zur Lehre. Wechselseitig können die Ergebnisse in die Systeme einfließen. Wir sind eng vernetzt mit zahlreichen Verbänden und Vereinen (z. B. DVW, DDGI, GEOkomm, GDI-Sachsen, GeoMV). 


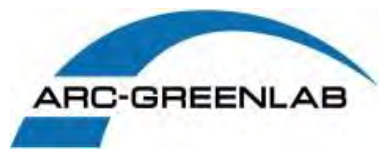

\section{ARC-GREENLAB GmbH}

12435 Berlin, Eichenstraße 3B

Telefon: 030/762 933 50, Fax: 030/762 93370

E-Mail: info@arc-greenlab.de

Internet: www.arc-greenlab.de

\section{ARC-GREENLAB STELLT SICH VOR}

Die wurde 1992 gegründet und ist ein interdisziplinäres Dienstleistungsunternehmen mit den Schwerpunkten Geoinformatik, Geodäsie und Gebäudemanagement. ARC-GREENLAB beschäftigt rund 95 Mitarbeiter an den Standorten Berlin und Hannover. Neben der Entwicklung und dem Vertrieb von Softwarelösungen bietet ARC-GREENLAB ein umfassendes Dienstleistungsspektrum. Die angebotenen Services umfassen die Durchführung von Kundenschulungen sowie die Erbringung von Beratungs-, Ingenieur- und Datendienstleistungen aus den Bereichen Vermessung, CAD, GIS, BIM, Flächen-, Infrastruktur-, und Facility Management sowie Forst und Umwelt. ARC-GREENLAB verfügt somit über die Gesamtkompetenz zur Durchführung anspruchsvollster Projekte von der Konzeption bis zur Realisierung

\section{LEISTUNGSSPEKTRUM}

ARC-GREENLAB entwickelt und vermarktet Fachanwendungen auf Basis von ArcGIS ${ }^{\circ}-$ Technologie für die Bereiche Vermessung, Kataster, Kommunal sowie Umwelt und Forst. ARC-GREENLAB steht für die Verwirklichung einheitlicher Lösungen für durchgängige Arbeitsprozesse im E-Government, beim Aufbau forstlicher Informations- und Managementsysteme sowie bei der Integration von Vermessung und GIS. Kunden von ARC-GREENLAB profitieren zudem von einem umfassenden Dienstleistungsangebot bei Beratung, Datenerfassung sowie Datenmigration und -aufbereitung. Unsere Arbeit ist geprägt durch kundenorientiertes Projektmanagement, motivierte und qualifizierte Mitarbeiter, schnelle Reaktionsfähigkeit, flexiblen Support, Mut zu unkonventionellen Lösungen und durch ein gewachsenes Netzwerk von Partnern.

\section{THEMENSCHWERPUNKTE}

Wir bieten Softwarelösungen und Dienstleistungen in folgenden Bereichen:

- Geoinformationssysteme, Datenaufbereitung und -migration im Bereich GIS und CAD 
- Werkzeuge zur Planerstellung und Visualisierung für XPlanung

- GIS-unterstützte Fachinformationssysteme für kommunale Infrastruktur (ALKIS, Baum, Grün, Spielplatz, Außenbeleuchtung, Friedhof), Bürgerportale

- Informations- und Managementsysteme für Forst- und Umwelt (Schutzgebiete, Biotope, FFH, Jagd, Forstbetrieb, Förderung)

- Kommunale- und Ingenieurvermessung, Dienstleistungen im BIMKontext (Bestandserfassung, Pläne, 3D-Modelle), Lösungen für CAFM, Instandhaltung, Liegenschaftsverwaltung und Bewirtschaftung

\section{REFERENZEN}

- GIS-unterstützte Fachinformationssysteme für kommunale Infrastruktur (Universitäts- und Hansestadt Greifswald, Stadtverwaltung Bergen, Landeshauptstadt Potsdam, Landkreise Potsdam-Mittelmark, TeltowFlämimg, Celle, Ilm-Kreis, ...)

- Umwelt- und Schutzgebietsmanagement (DBU Naturerbe GmbH, Nationalpark Kellerwald, Naturpark Drömling und Südharz, Naturschutzpark Lüneburger Heide, Landesamt für Umwelt Brandenburg, ...)

- Kampfmittelmanagement (Kampfmittelflächenkataster der Hamburger Feuerwehr, GIS-gestütztes Dokumentationssystem beim Technischen Polizeiamt des Landes Sachsen-Anhalt)

- XPlanung (Hansestadt Hamburg, Stadt Dessau-Roßlau, Landgesellschaft Sachsen-Anhalt, ...)

- Anwendung für Forstbetriebe und Jagdmanagement (Landesbetrieb Forst Brandenburg, Berliner Forsten, Stadtforst Frankfurt am Main, Landesforstbetrieb Sachsen-Anhalt, Landwirtschaftskammer Niedersachsen, ...)

- Kommunale- und Ingenieurvermessung, Bestandserfassung, Datendienstleistungen (Deutsche Bahn, Berliner Verkehrsbetriebe, Berliner Schloss/Humboldt-Forum, Berliner Wasserbetriebe, edis AG, Mittelmärkische Wasser- und Abwasser $\mathrm{GmbH}, . .$.

Lösungen für CAFM, Instandhaltung, Liegenschaftsverwaltung und Bewirtschaftung (Landkreise in Mecklenburg-Vorpommern, wie z. B. VorpommernRügen und Mecklenburgische Seenplatte, Stadt Greifswald, Stadt Stralsund, Neubrandenburg, Wohnbau GmbH Prenzlau, Studentenwerk OstNiedersachsen, Caritas Altenhilfe GGmbH, ...) 


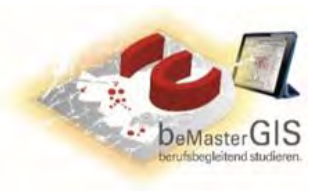

Hochschule Anhalt, FB 3, IGV

06846 Dessau-R., Bauhausstraße 8

Telefon: 0340/51971573, Fax: 0340/5197/3733

E-Mail: master-gis@afg.hs-anhalt.de

Internet: www.beMasterGIS.de

\section{ONLINE-MASTERSTUDIENGANG „BEMASTERGIS“}

Aufgrund der rasend schnellen technischen Entwicklung verspüren viele Fachanwender von Geoinformationssystemen (GIS) den Wunsch, hier eine dezidierte Ausbildung vorzunehmen. Deshalb wurde im Jahre 2010 der OnlineMasterstudiengang Geoinformationssysteme an der Hochschule Anhalt (Campus Dessau) aus der Taufe gehoben. Angesprochen fühlen sich Anwender von Geoinformationssystemen, die in der kommunalen Verwaltung, im Planungsbereich, im Umwelt- und Naturschutz, in der Versorgungswirtschaft, im Marketing und anderen Bereichen arbeiten oder die Verbindung zu GIS mit ihrem persönlichen Arbeitsumfeld planen. Das fünfsemestrige Fernstudium entspricht in Qualität, Umfang und Wertigkeit einem Direktstudium.

Charakteristisch für diesen Online-Weiterbildungsstudiengang ist der hohe Anteil an betreutem Selbststudium (90\% der Studieninhalte sind internetfähig aufbereitet). Die Teilnehmer studieren über eine moderne Lernplattform, unabhängig von Hörsaal und Lehrveranstaltungen ganz nach ihren individuellen Bedingungen. Das Lerntempo und die Intensität bestimmen sie während der Selbstlern-phasen überwiegend selbst. Diese werden pro Semester zweimal durch Präsenz-phasen an je einem Wochenende unterbrochen. Die derzeit über 65 eingeschriebenen Studierenden kommen aus dem gesamten Bundesgebiet, einige sogar aus der Schweiz und Frankreich. Das Durchschnittsalter beträgt etwa 32 Jahre. Und obwohl in den Ingenieurwissenschaften eher weniger weibliche Beschäftigte arbeiten, studieren in diesem Studiengang ca. $40 \%$ Frauen. Interessierte werden für das Online-Masterstudium GIS zugelassen, wenn sie einen ersten akademischen Abschluss sowie mindestens ein Jahr Berufserfahrung im Umfeld von Geoinformationssystemen nachweisen. Der Studienbeginn ist jeweils Ende September eines jeden Jahres.

Weitere Informationen zum Studium finden Sie hier: http://www.bemastergis.de/ 


\section{STUDIENVORAUSSETZUNGN}

Ein qualifizierter Hochschulabschluss in einem Bachelor- oder Diplomstudiengang mit einer Regelstudienzeit von mindestens sieben Semestern (sechs Semes-ter möglich bei Belegung von Zusatzmodulen) sowie eine darauf aufbauende qualifizierte berufspraktische Erfahrung nicht unter einem Jahr.

Die Zulassung erfolgt nach einem Feststellungsverfahren.

\section{STUDIENSCHWERPUNKTE}

- Grundlagen und Anwendung von GIS

- Fernerkundung

- Mathematische Methoden in Geodäsie und GIS

- Modellierung und Analyse

- Visualisierung von Geodaten

- Datenbanken und Geodatenbanken

- Kartografie

- Geodateninfrastrukturen

- Wahlpflichtmodule, so beispielsweise: Raum- und Umweltplanung, Projektmanagement, Führungsqualifikation, Web Mapping, multisensorale Fernerkundungsanalyse 


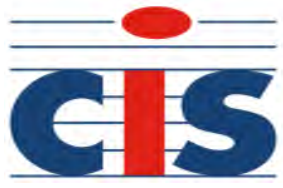

\section{$\mathrm{CiS} \mathrm{GmbH}$}

18182 Bentwisch, Hansestraße 21

Telefon: 0381/87399700, Fax: 0381/87399730

E-Mail: info@cis-rostock.de

Internet: cis-rostock.de

\section{DAS UNTERNEHMEN STELLT SICH VOR}

Die CiS GmbH beschäftigt sich mit Raumbezogenen Informationssystemen für verschiedene Anwendungsgebiete. Die seit 1990 bestehende Firma entwickelt und betreibt Geografische Informationssysteme für Leitungsbetreiber, landwirtschaftliche und industrielle Firmen und Behörden für Ämter und Kommunen.

Einer der neueren Geschäftszweige ist die Herstellung von unbemannten Fluggeräten und der zugehörigen Software für Vermessung und Inspektion. Bearbeitet werden auch Lösungen für Landwirtschaft und Umweltschutz.

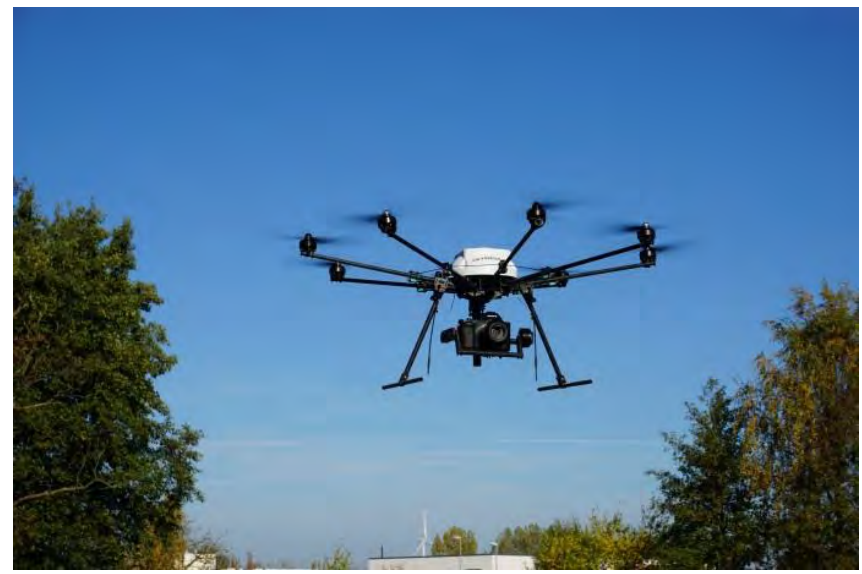

Die UAS können sowohl outdoor mit GPS als auch indoor eingesetzt werden.

CiS ist seit ca. 10 Jahren in diesem Geschäftsfeld tätig und kann Lösungen mit hoher Effizienz für die Endnutzer bereitstellen. Auf Wunsch wird auch Pflege und Aufbereitung der Daten angeboten. Lehrgänge für die Anwender einschließlich Prüfung zum Erwerb des Kenntnisnachweises runden unser Angebot ab. 


\section{LEISTUNGSSPEKTRUM}

Von Modellierung, Programmierung, Ersterfassung bis Inspektion von Netzinformationssystemen und anderen GIS-Systemen bis hin zur Entwicklung spezieller Hard- und Software nach Kundenwunsch.

\section{THEMENSCHWERPUNKTE}

- Netzinformationssysteme für Leitungsbetreiber wie Stadtwerke Rostock, Mainz, Bremen.

- UAS und Auswertungssoftware für 2D- und 3D-Vermessung, Inspektion von Gebäuden und industriellen Anlagen.

- Entwicklung von KI-Lösungen für den Einsatz auf UAS zur Erarbeitung von autonomen Inspektionssystemen und als Knoten in nomadischen Netzen.

\section{REFERENZEN}

\begin{tabular}{|c|l|l|l|}
\hline 5 & $\begin{array}{l}\text { Entwicklung von Netzinformationssystem- } \\
\text { komponenten für verschiedene Sparten von } \\
\text { Leitungsbetreibern (Fernwärme, Gas, } \\
\text { Strom, Kommunikation, ...) }\end{array}$ & $\begin{array}{l}\text { Stadtwerke Rostock } \\
\text { Stadtwerke Mainz } \\
\text { Stadtwerke Bremen }\end{array}$ & $\begin{array}{l}1994- \\
2019\end{array}$ \\
\hline 5 & $\begin{array}{l}\text { Befliegung diverser Kreuzungen zwecks } \\
\text { Kartierung der Straßenmarkierungen }\end{array}$ & Stadtwerke Rostock & $\begin{array}{l}2018- \\
2019\end{array}$ \\
\hline 6 & $\begin{array}{l}\text { Befliegung 12 km Gasleitung anstelle von } \\
\text { Begehungen, Orthofotoerstellung }\end{array}$ & Stadtwerke Rostock & 2014 \\
\hline 2 & $\begin{array}{l}\text { Testbefliegung für Gewässerrenaturierung, } \\
\text { 3D-Vermessung und Modellierung, Kopter, } \\
\text { Software, Bodenstation und Lieferung } \\
\text { Bildverarbeitungsrechner }\end{array}$ & $\begin{array}{l}\text { Abteilung Geoinformati- } \\
\text { on, Kataster und Vermes- } \\
\text { sung, Kreishaus Gütersloh } \\
\text { 33324 Gütersloh }\end{array}$ & $\begin{array}{l}2016- \\
2019\end{array}$ \\
\hline 3 & $\begin{array}{l}\text { Befliegung, Modellierung und Volumenbe- } \\
\text { rechnung, ca. 60 ha dezimetergenau }\end{array}$ & Deponie Rosenow & $\begin{array}{l}2015- \\
2019\end{array}$ \\
\hline
\end{tabular}




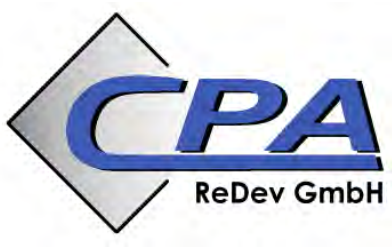

CPA ReDev GmbH

53721 Siegburg, Auf dem Seidenberg 3a

Telefon: 02241/25940, Fax: 02241/259429

E-Mail: mail@supportgis.de

Internet: http://www.cpa-redev.de

\section{DAS UNTERNEHMEN STELLT SICH VOR}

Die CPA ReDev GmbH ist ein aus der CPA Geo-Information im Jahr 2013 hervorgegangenes Software-Unternehmen der Geoinformationswirtschaft mit nationalen und internationalen Tätigkeitsfeldern.

Das Unternehmen ist in Siegburg ansässig. Es führt die Unternehmensstrategie der CPA nahtlos fort, für aktuelle geowissenschaftliche Fragestellungen moderne, normenkonforme und datenbankgestützt arbeitende Technologien in den Bereichen

- OpenGIS- und ISO-konforme nD-Datenbank- und Client-Lösungen im Intranet und Internet,

- 3D-Stadt- und Landschaftsmodelle,

- Mobile GIS für den Außendienst,

- Führung des Amtlichen Liegenschaftskatasters (ALKIS®),

- Informationssysteme für die Forstverwaltung,

- eRobotik für weltraumnahe Forschungsvorhaben

anzubieten. Das Unternehmen stellt dazu mit SupportGIS eine Basistechnologie für ein ISO-konformes Datenmanagement zur Verfügung und setzt diese Plattform und das darüber erworbene Know-how ebenso erfolgreich in seinem Projektgeschäft ein.

Es ist das Bestreben der CPA, mit innovativen Lösungen jeweils an der technologischen Spitze des Marktsegmentes der Geoinformationswirtschaft zu stehen. Die folgenden Produktlinien stehen für diesen Einsatz:

- SGJ-3D

- $\quad$ SGJ-Forsten

- SGJ-ALKIS
3D-Stadtmodelle

Forstinformationssysteme

Amtliches Liegenschaftskataster 
- $\quad$ SGJ-GeoHornet

- SGJ-Data Provider
Webbasiertes Internet-GIS

GeoCloud für die Geodatenversorgung

\section{LEISTUNGSSPEKTRUM}

Die CPA ReDev GmbH ist ein Software-Unternehmen der GIS-Branche. Es ist hochspezialisiert auf die Entwicklung von Software, die überwiegend im $\mathrm{Zu}-$ sammenhang steht mit der Bewältigung und Führung von großen bis sehr großen Geodatenbeständen. Dazu werden mehrdimensionale und datenbankgestützt arbeitende Programmsysteme mit bis zu drei Zeitebenen entwickelt, die hochkomplexe und auch sicherheitskritische Anforderungen im Bereich der Datenbereitstellung, der Daseinsvorsorge und dem Klimaschutz anwendungsbezogen und kundenspezifisch umsetzen.

\section{THEMENSCHWERPUNKTE}

Schwerpunkte der Entwicklung sind Programmsysteme mit komplexen Datenstrukturen und großen Datenvolumina. Stellvertretend dafür stehen Anwendungen aus den Bereichen 3D-Stadtmodelle (CityGML), Amtliches Liegenschaftskataster (ALKIS), forstliche Großrauminventur- und Planungssysteme (ForestGML) und die Verwaltung weltweit verfügbarer Topografiedaten in verschiedenen Dimensionen, Auflösung bzw. Detaillierungsgraden.

Diese Programmsysteme stehen dem Kunden als Software-Produkte im Intranet und Internet zur Verfügung. Sie werden im Rahmen von Entwicklungsprojekten an dessen Bedürfnisse individuell angepasst und nachhaltig betreut. Aufgrund des innovativen Ansatzes der SupportGIS-Technologie zur Verwaltung raum-, sach- und zeitbezogener Datenbestände kommt diese Technologie in immer größerem Umfang auch in universitären Forschungsprojekten zum Einsatz.

\section{REFERENZEN}

- Bundesamt für Ausrüstung, Informationstechnik und Nutzung der Bundeswehr (BAAINBw)

- Bundesland Mecklenburg-Vorpommern (Anwendungen: ALKIS, 3D)

- Bundesland Baden-Württemberg (Anwendung: ALKIS)

- RWTH Aachen (Anwendung: eRobotik, Virtuelle Testbeds) 


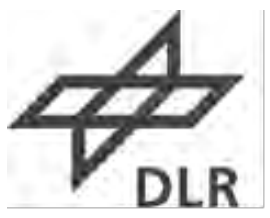

Deutsches Zentrum für Luft- und Raumfahrt

17235 Neustrelitz, Kalkhorstweg 53

Telefon: 03981/480 116, Fax: 03981/480299

E-Mail: egbert.schwarz@dlr.de

Internet: www.dlr.de

\section{DLR - STANDORT NEUSTRELITZ}

\section{STELLT SICH VOR}

Am DLR-Standort Neustrelitz konzentrieren sich die Forschungs- und Entwicklungsarbeiten auf die Themen Satellitendatenempfang, Satellitenfernerkundung, Navigation, Weltraumwetter sowie maritimer Verkehr und maritime Sicherheit. Etwa 80 Mitarbeiterinnen und Mitarbeiter sind dazu an den Instituten Deutsches Fernerkundungsdatenzentrum DFD, Institut für Methodik der Fernerkundung IMF, Institut für Kommunikation und Navigation IKN sowie Institut für SolarTerrestrische Physik SO tätig. Im Rahmen der Nachwuchsförderung betreibt das DLR am Standort zudem ein School_Lab.

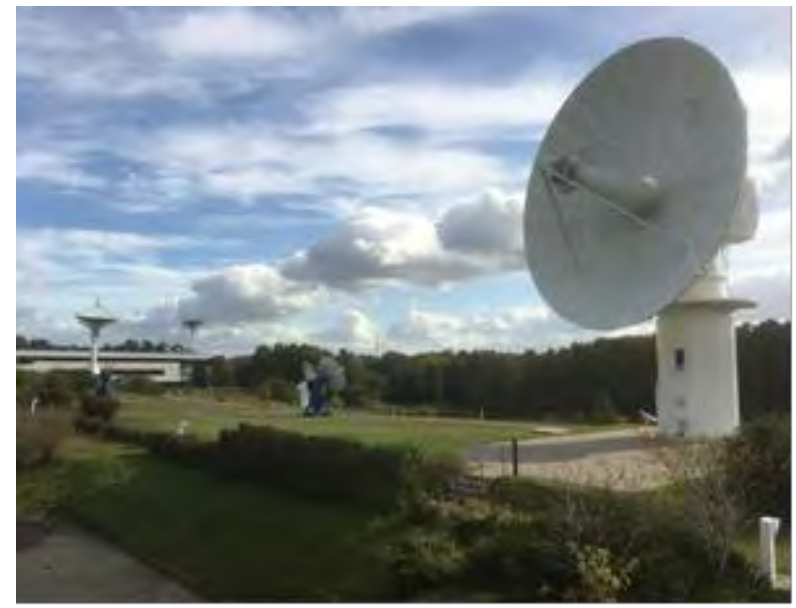

Die Globalisierung stellt uns vor immer neue Herausforderungen - so auch für die Sicherheit der Schifffahrt und den Schutz der Meere. Dazu hat das DLR am Standort Neustrelitz eine von vier Forschungsstellen zur maritimen Sicherheit etabliert, um hier die institutsübergreifenden Kompetenzen des Deutschen Fern- 
erkundungsdatenzentrums und des Instituts für Kommunikation und Navigation in den Bereichen satellitengestützte Echtzeitsysteme und maritime Verkehrstechnik zu bündeln. Ziel der gemeinsamen Arbeit der Forschungsstelle ist die Entwicklung und Erprobung von Technologien zur Gewährleistung sicherer Schifffahrtsrouten, zum Schutz der Meere und Küstengewässer sowie zur Unterstützung von Behörden bei der Bekämpfung illegaler Aktivitäten wie zum Beispiel Verklappung von Öl, illegaler Fischerei und Piraterie. Seitens des DFD arbeitet das Team der Forschungsstelle Maritime Sicherheit Neustrelitz an der Entwicklung und Validierung von Echtzeitsystemen zur Generierung von maritimen Informationsprodukten aus Satellitendaten, sowie deren Bereitstellung und Verteilung an die Nutzer.

\section{THEMENSCHWERPUNKTE DER FORSCHUNGSSTELLE MARITIME SICHERHEIT IM DFD}

Die Forschungs- und Entwicklungsaufgaben der DLR-Forschungsstelle Maritime Sicherheit Neustrelitz, Teil DFD, lassen sich wie folgt zusammenfassen:

- Schaffung der Systemvoraussetzungen zum Empfang neuer Missionen (Team Bodenstationen)

- Multisensorale Basisprozessierung

- Datenfusionierung (z. B. SAR-Daten, optische Erdbeobachtungsdaten und AIS)

- Entwicklung maritimer Informationsprodukte

- Entwicklung von Systemen zur Produktbereitstellung, sowie die Schnittstellenentwicklung entsprechend der Nutzeranforderungen

- Entwicklung von Systemkomponenten zur Echtzeitprozessierung von Satellitendaten

- Prozessorintegration und Validierung

\section{REFERENZEN}

- Copernicus, Nationales „Collaborative Ground Segment“ Mission Sentinel-1

- Copernicus, Maritime Surveillance Services in Zusammenarbeit mit European Space Imaging EUSI aus München.

- Earth Observation Maritime Surveillance System (EO-MARISS) 


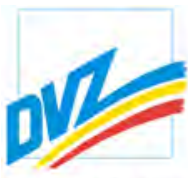

DVZ Datenverarbeitungszentrum Mecklenburg-Vorpommern $\mathrm{GmbH}$
DVZ Datenverarbeitungszentrum M-V GmbH

19059 Schwerin, Lübecker Straße 283

Telefon: 0385/48000, Telefax: 0385/4800487

E-Mail: marketing@dvz-mv.de

Internet: www.dvz-mv.de

\section{DVZ STELLT SICH VOR}

Die DVZ M-V GmbH ist der IT-Service-Provider der Landesverwaltung Mecklenburg-Vorpommern mit Sitz in Schwerin. Unsere mehr als 550 hochqualifizierten Mitarbeiterinnen und Mitarbeiter nutzen täglich verschiedenste Kompetenzen, um Verwaltungs-Know-how mit zukunftsorientierter Informations- und Kommunikationstechnologie zu verbinden. Denn als langjähriger Partner des öffentlichen Sektors stehen wir gemeinsam vor der Herausforderung, die Verwaltung mit modernsten IT-Lösungen auf dem Weg zum Rund-um-die-Uhrerreichbaren Bürgerdienstleister zu begleiten.

Dabei haben Anforderungen nach höchstmöglicher Sicherheit, uneingeschränktem Daten-schutz und permanenter Verfügbarkeit für unser Handeln oberste Priorität. Sie sind Maßstab für die Entwicklung zukunftsweisender, durchgängig vernetzter und medienbruchfrei-er Dienste, aber auch für den Betrieb des eigenen Rechenzentrums. Consulting- und Compliance-Leistungen gehören ebenso zu unseren Kernkompetenzen wie der Betrieb sicherer Kommunikationsinfrastrukturen oder die Entwicklung eigener Applikationen, Dienste und Servicemodelle. So sind durch uns entwickelte, betreute und betriebene Fachapplikationen beispielsweise in den Bereichen Justiz, Innere Sicherheit, Personenstandswesen oder Geoinformation vollumfänglich in die Arbeit der Verwaltung integriert und in einer zu-nehmend mit dem Bürger vernetzten Verwaltung nicht mehr wegzudenken.

Unsere Kernkompetenzen liegen unter anderem in den Geschäftsfeldern:

- It-Consulting

- It-Compliance und Security

- Fachapplikationen

- Managed Services

- Sicherheitsinfrastrukturen

- Rechenzentrum

- Zentrale Beschaffung, Technischer Service, Seminare und Trainings 


\section{LEISTUNGSSPEKTRUM BEREICH GEOINFORMATION}

- Aufbau und Betrieb von Geodateninfrastrukturen

- Konzeption und Entwicklung von WebGIS-Fachanwendungen für verschiedenste Fachgebiete

- Betrieb und Betreuung von vernetzten Geoinformationssystemen und Geoservern und deren Fachanwendungen

- Schulung und Beratung zu Geoinformationssystemen und -themen

- Mitarbeit in Vereinen und Netzwerken der Geoinformationswirtschaft $\mathrm{M}-\mathrm{V}$

\section{THEMENSCHWERPUNKTE}

- Betrieb und Weiterentwicklung der Geodateninfrastruktur M-V

- GeoPortal.MV

- Metainformationssystem

- GAIA-MVlight und GAIA-MVprofessional

- GeoWebDienste nach OGC, GDI-DE und INSPIRE

- Sicherheits- und Abrechnungsstrukturen

- Vernetzung mit anderen Geodateninfrastrukturen

- Entwicklung und Betrieb von WebGIS-Fachapplikationen

- Lösung (API) zur Integration von Geodaten in Web-Präsentationen

- Betrieb und Betreuung der zentralen Datenbanken für Geobasisdaten (ALKIS, ATKIS, AFIS)

- Aufbereitung und Abgabe von Geodaten an Nutzer

\section{REFERENZEN (AUSWAHL)}

- Landesamt für innere Verwaltung $\mathrm{M}-\mathrm{V}$

- Landesforst Mecklenburg-Vorpommern

- Ministerium für Wirtschaft

- Landesamt für Straßenbau und Verkehr

- Landgesellschaft Mecklenburg-Vorpommern mbH 


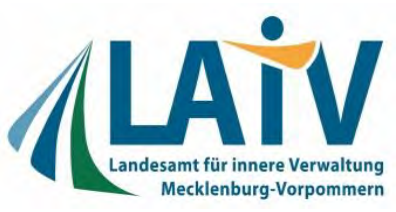

LAiV M-V / AFGVK Amt für Geoinformation, Vermessungs- und Katasterwesen

19059 Schwerin, Lübecker Str. 289

Telefon: $0385 / 58856860$

E-Mail: geodatenservice@laiv-mv.de

Internet: www.laiv-mv.de

\section{AFGVK M-V STELLT SICH VOR}

Das Amt für Geoinformation, Vermessungs- und Katasterwesen (AfGVK) im Landesamt für innere Verwaltung ist die für das amtliche Vermessungswesen des Landes zuständige obere Vermessungs- und Geoinformationsbehörde. Aufgabe des amtlichen Vermessungswesens ist es, die Geobasisdaten für die Landesfläche zu erheben und landesweit nachzuweisen.

Geobasisdaten beschreiben die Erscheinungsform der Erde (Topographie) und die Liegenschaften (Flurstücke und Gebäude) mit ihren grundstücksgleichen Rechten. Sie sind in einem einheitlichen Raumbezug definiert und haben für die vielfältigen Bedürfnisse von Politik, Verwaltung und Wirtschaft eine herausragende Bedeutung. Geobasisdaten werden u. a. für die Erhebung, den Nachweis und die Präsentation von Geofachdaten benötigt.

Die Topographie der Erdoberfläche wird im Amtlichen TopographischKartographischen Informationssystem (ATKIS) geführt und für vielfältige Nutzungen angeboten. Bestandteile von ATKIS sind neben den Digitalen Landschaftsmodellen (DLM) und den Digitalen Geländemodellen (DGM) auch die Digitalen Oberflächenmodelle (DOM), die Digitalen Orthofotos (DOP) und die Digitalen Topographischen Karten (DTK).

Die Daten über Liegenschaften werden im Amtlichen LiegenschaftskatasterInformationssystem (ALKIS) geführt.

Die Geobasisdaten sind Teil der Geodateninfrastruktur MecklenburgVorpommerns (GDI-MV). 
Das Amt ist darüber hinaus Aufgabenträger unter anderem der:

- Geschäftsstelle des Prüfungsausschusses für das 1. Einstiegsamt der Laufbahngruppe 2 des technischen Dienstes im Bereich Vermessungswesen und Zuständige Stelle nach dem Berufsbildungsgesetz für Geomatiker und Vermessungstechniker,

- Geschäftsstelle des Oberen Gutachterausschusses für Grundstückswertermittlung,

- Koordinierungsstelle für das Geoinformationswesen,

- Fachaufsicht über die Vermessungsstellen. 


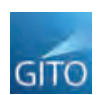

Business Analytics Big Data

Cloud Computing

CRM - Coustomer

Relationship Management

Digitalisierung

ECM - Enterprise Content

Management

Electronic Business
Energieeffizienz

ERP - Enterprise Resource Planning

Fabrikmanagement

Fertigungsmanagement

Fertigungssteuerung

Geschäftsprozessmanagement

Industrie 4.0

IT-Architektur

Lean Production
Materialmanagement

MES - Manufacturing Execution

Systeme

Produktentwicklung

Produktionssysteme

Qualitätsmanagement

Qualifizierung

Safety and Security

Simulation
Software Engeneering

Supply Chain Management

Unternehmensführung und

Organisation

Usability

Wirtschaftsinformatik

Wissensmanagement

\section{Unternehmensführung und Organisation}

8326 Scholz-Reiter, B. Autonome Systeme (Industrie Management 1/2011)

8327 Gronau, N.

8328 Gronau, N.

8330 Scholz-Reiter, B.

8332 Bill, R.,

Flach, G.,

Klammer, U.,

Lerche, T. (Hrsg.)

8334 Heine, Moreen

8339 Gronau, N.;

Meier, Horst;

Bahrs, Julian

(Hrsg.)

8342 Gronau, N.

8345 Bentele, Markus;

Gronau, N.;

Schütt, Peter;

Weber, Mathias

(Hrsg.)

8353 Stracke, Christian M.

8355 Gronau, N.

8358 Soelberg, Christian

8360 Gronau, N.

8361 Borg, Erik;

Daedelow,

Holger;

Johnson, Ryan

(Hrsg.)

8370 Gronau, N.

8371 Gronau, N.

8374 Müller, Egon

(Hrsg.)

8375 Gronau, N.;

Weber, Nadja;

Jähnchen, Marie

Mobiles Arbeiten und Sicherheit (iSuccess 1/2011)

Effizienz durch ERP (ERP Management 1/2011)

Brasilien (Industrie Management 2/2011)

GeoForum MV 2011 - Geodateninfrastrukturen: Drehscheibe für Wirtschaft und Verwaltung

Transfer von E-Government-Lösungen - Wirkungen und Strategien

Handbuch gegen Produktpiraterie - Prävention von

Produktpiraterie durch Technologie, Organisation und

Wissensflussmanagement

Erfolgsfaktor Personal (Industrie Management 4/2011)

KnowTech - Unternehmenswissen als Erfolgsfaktor mobilisieren!

Competence Modelling for Human Resources Development and European Policies

ERP-Strategien (ERP Management 4/2011)

Wissenskapital als Instrument der strategischen Unternehmensführung

Wettbewerbsfähigkeit (ERP Management 1/2012)

RapidEye Science Archive (RESA) - Vom Algorithmus zum Produkt
Customer Relationship Management (ERP Management 2/2012)

Demographischer Wandel - Herausforderung für die Arbeits- und Betriebsorganisation der Zukunft

Wettbewerbsfaktor Analytics - Status, Potenziale, Herausforderung

$978-3-942183-26-0$

66

978-3-942183-27-7

82

$978-3-942183-28-4$

82

978-3-942183-30-7

$978-3-942183-32-1$

82

$978-3-942183-42-0$

610 978-3-942183-45-1

$978-3-942183-53-6$

66

$978-3-942183-60-4$

232 978-3-942183-61-1

82

66

978-3-942183-70-3

978-3-942183-71-0

461

978-3-942183-74-1

164

978-3-942183-75-8 


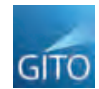

Business Analytics

Big Data

Cloud Computing

CRM - Coustomer

Relationship Management

Digitalisierung

ECM - Enterprise Content

Management

Electronic Business
Energieeffizienz

ERP - Enterprise Resource Planning

Fabrikmanagement

Fertigungsmanagement

Fertigungssteuerung

Geschäftsprozessmanagement

Industrie 4.0

IT-Architektur

Lean Production
Materialmanagement

MES - Manufacturing Execution

Systeme

Produktentwicklung

Produktionssysteme

Qualitätsmanagement

Qualifizierung

Safety and Security

Simulation
Software Engeneering

Supply Chain Management Unternehmensführung und

Organisation

Usability

Wirtschaftsinformatik

Wissensmanagement
8378 Gronau, N. (Hrsg.);

Thim, Christof;

Röchert-Voigt,

Tanja; Proske,

Niels;

Heine, Moreen;

Korte, Edgar

8382 Bentele, Markus;

Gronau, N.;

Schütt, Peter;

Weber, Mathias

(Hrsg.)

8386 Stracke, Christian M.

8391 Gronau, N.

8393 Gronau, N.

8394 Gronau, N.

5010 Gronau, N.

5016 Lehner, Franz;

Amende, Nadine;

Fteimi, Nora

(Hrsg.)

5021 Gronau, N.

5025 Scholz-Reiter, B.; Krohne, Farian

5029 Gronau, N.

5036 Gronau, N.; Eggert, S.

5038 Gronau, N.

5042 Lee, Seung-Ho

5050 Biedermann, Hubert (Hrsg.)

5051 Gronau Norbert; Weber, Nadja; Fohrholz, Corinna

5056 Mehrsai, Afshin

5057 Gronau, N.; Scholz-Reiter, B.
Organisation des Schutzes der Kritischen Infrastruktur

Wasserversorgung - Grundlagen und praktische Anwendung für Betreiber

KnowTech - Neue Horizonte für das Unternehmenswissen - Social Media, Collaboration, Mobility

662

$978-3-942183-82-6$

The Future of Learning Innovations and Learning Quality -

$978-3-942183-86-4$

How do they fit together?

ERP-Markt.info 2/2012

$978-3-942183-91-8$

Hidden Champions (Industrie Management 6/2012)

66

$978-3-942183-93-2$

Prozessmanagement mit ERP (ERP Management 4/2012)

66

$978-3-942183-94-9$

Wirtschaftlichkeit (ERP Management 1/2013)

66

978-3-95545-010-6

Konferenzbeiträge der 7. Konferenz Professionelles Wis-

sensmanagement

$978-3-95545-016-8$

Demografische Veränderung der Arbeitswelt (Industrie Management 3/2013)

$978-3-95545-021-2$

Entwicklung einer Bewertungsmethode für das Anlaufmanagement (Informationstechnische Systeme und Organisation von Produktion und Logistik, Band 15)

978-3-95545-025-0

Cloud Computing (Industrie Management 4/2013)

ERP Add-ons (ERP Management 3/2013)

Eco-Innovation (Industrie Management 5/2013)

Ansatz zur Erhöhung der Produktivität durch Wissen: Unter Berücksichtigung von kulturellen Aspekten, Produkt- und Prozess-Komplexität

Corporate Capability Management - Wie wird kollektive

978-3-95545-050-2 Intelligenz im Unternehmen genutzt?

Forschungsstudie 2013 - Wettbewerbsfaktor Analytics (E-Book)

Feasibility of Autonomous Logistic Processes Introduction

242

978-3-95545-056-4 of Learning Pallets

30 Jahre industrielle Geschäftsprozesse (Industrie Manage- 66 ment 1/2014) 


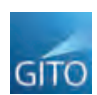

Business Analytics Big Data

Cloud Computing

CRM - Coustomer

Relationship Management

Digitalisierung

ECM - Enterprise Content

Management

Electronic Business
Energieeffizienz

ERP - Enterprise Resource Planning

Fabrikmanagement

Fertigungsmanagement

Fertigungssteuerung

Geschäftsprozessmanagement

Industrie 4.0

IT-Architektur

Lean Production
Materialmanagement

MES - Manufacturing Execution

Systeme

Produktentwicklung

Produktionssysteme

Qualitätsmanagement

Qualifizierung

Safety and Security

Simulation
Software Engeneering

Supply Chain Management

Unternehmensführung und

Organisation

Usability

Wirtschaftsinformatik

Wissensmanagement
5060 Eggert, S., S. Norbert Gronau

5076

Gronau, N.

5087 Gronau, N.

5100 Gronau, N.

5123 Röchert-Voigt, Tanja, Gronau, N. (Hrsg.)

5128

Meier, Horst (Hrsg.)

5145

5150 Gronau, N.

5158 Gronau, N.

5170 Gronau, N.

5172 Gronau, N.

5176 Scholz-Reiter, B.

5174 Gronau, N.

5185 Christopher M. Schlick (Hrsg)

5184 G.

5213 Gronau, N.

5239 Gronau, N.; Eggert, $\mathrm{S}$.

5218

5222

5225

5223

Gronau, N.

Klarenbach, I.
92 ERP-Systeme im Vergleich - Mobile ERP Funktionen

Trends 2014 (ERP Marktüberblick 1/2014)

CRM (ERP Management 2/2014)

Business Analytics (ERP Management 3/2014)

Manufacturing Analytics (Productivity Management 5/2014)

Gubernative Rechtsetzung mit Social Software

Lehren und Lernen für die moderne Arbeitswelt

Erarbeitung einer Methodik der Wandlungsfähigkeit

Geschäftsprozessmanagement in Wirtschaft und Verwaltung - Analyse, Modellierung und Konzeption

ERP der Zukunft (ERP Management 1/2016)

ERP-Verträge (ERP Management 2/2016)

66

66

$978-3-95545-100-4$

$978-3-95545-123-3$

$978-3-95545-128-8$

978-3-95545-145-5

249

978-3-95545-150-9

66

978-3-95545-158-5

64

$978-3-95545-170-7$

Qualifizierung für die Industrie 4.0 (Industrie 4.0 Management 3/2016)

Industrie 4.0 Forschungs- und Anwendungszentren (Industrie 4.0 Management 4/2016)

$978-3-95545-176-9$

Industrie 4.0 (productiviTy 3/2016)

64

$978-3-95545-174-5$

$978-3-95545-185-1$

Megatrend Digitalisie
Betriebsorganisation

$978-3-95545-184-4$

64

$978-3-95545-213-1$

66

$978-3-95545-239-1$

4/2017)

64

$978-3-95545-218-6$

418

$978-3-95545-222-3$ tionsansatz

Gronau, N. (Hrsg.)

Industrial Internet of Things in der Arbeits- und Betriebsorganisation

260

$978-3-95545-225-4$

Vladova, Gergana

Wissensmanagement im Kontex der Interdependenzen zwischen Unternehmenskultur und Beruflicher kultureller Prägung

286

$978-3-95545-223-0$ Management 2/2019) 